\title{
THE GEOGRAPHY OF DEVELOPMENT: \\ EVALUATING MIGRATION RESTRICTIONS AND COASTAL FLOODING
}

\author{
Klaus Desmet \\ Dávid Krisztián Nagy \\ Esteban Rossi-Hansberg \\ Working Paper 21087 \\ http://www.nber.org/papers/w21087
}

\author{
NATIONAL BUREAU OF ECONOMIC RESEARCH \\ 1050 Massachusetts Avenue \\ Cambridge, MA 02138 \\ April 2015
}

We are grateful to the Gallup Organization and to Angus Deaton for providing us with aggregate national data on wellbeing and on preferred migration destinations from the Gallup World Poll. We also thank Treb Allen, Maya Buchanan, Angus Deaton, Tom Holmes, Chang-Tai Hsieh, Erik Hurst, Steve Redding, Frédéric Robert-Nicoud, Jesse Shapiro, Joe Shapiro, and Rob Townsend for comments and discussions. The views expressed herein are those of the authors and do not necessarily reflect the views of the National Bureau of Economic Research.

NBER working papers are circulated for discussion and comment purposes. They have not been peerreviewed or been subject to the review by the NBER Board of Directors that accompanies official NBER publications.

(C) 2015 by Klaus Desmet, Dávid Krisztián Nagy, and Esteban Rossi-Hansberg. All rights reserved. Short sections of text, not to exceed two paragraphs, may be quoted without explicit permission provided that full credit, including $(\odot$ notice, is given to the source. 
The Geography of Development: Evaluating Migration Restrictions and Coastal Flooding Klaus Desmet, Dávid Krisztián Nagy, and Esteban Rossi-Hansberg

NBER Working Paper No. 21087

April 2015

JEL No. E2,F11,F18,F22,F43,O1,O4,R23

\begin{abstract}
$\underline{\text { ABSTRACT }}$
We study the relationship between geography and growth. To do so, we first develop a dynamic spatial growth theory with realistic geography. We characterize the model and its balanced growth path and propose a methodology to analyze equilibria with different levels of migration frictions. We bring the model to the data for the whole world economy at a $1^{\circ} \times 1^{\circ}$ geographic resolution. We then use the model to quantify the gains from relaxing migration restrictions as well as to describe the evolution of the distribution of economic activity in the different migration scenarios. Our results indicate that fully liberalizing migration would increase welfare more than three-fold and would significantly affect the evolution of particular regions in the world. We then use the model to study the effect of a spatial shock. We focus on the example of a rise in the sea level and find that coastal flooding can have an important impact on welfare by changing the geographic-dynamic path of the world economy.
\end{abstract}

Klaus Desmet

SMU

Department of Economics

3300 Dyer, Suite 301

Dallas, TX 75205

kdesmet@smu.edu

Dávid Krisztián Nagy

Princeton University

Department of Economics

Fisher Hall

Princeton, NJ 08544-1021

dnagy@exchange.Princeton.EDU
Esteban Rossi-Hansberg

Princeton University

Department of Economics

Fisher Hall

Princeton, NJ 08544-1021

and NBER

erossi@princeton.edu 


\title{
The Geography of Development: Evaluating Migration Restrictions and Coastal Flooding*
}

\author{
Klaus Desmet \\ $S M U$
}

\author{
Dávid Krisztián Nagy \\ Princeton University
}

\author{
Esteban Rossi-Hansberg \\ Princeton University
}

April 3, 2015

\begin{abstract}
We study the relationship between geography and growth. To do so, we first develop a dynamic spatial growth theory with realistic geography. We characterize the model and its balanced growth path and propose a methodology to analyze equilibria with different levels of migration frictions. We bring the model to the data for the whole world economy at a $1^{\circ} \times 1^{\circ}$ geographic resolution. We then use the model to quantify the gains from relaxing migration restrictions as well as to describe the evolution of the distribution of economic activity in the different migration scenarios. Our results indicate that fully liberalizing migration would increase welfare more than three-fold and would significantly affect the evolution of particular regions in the world. We then use the model to study the effect of a spatial shock. We focus on the example of a rise in the sea level and find that coastal flooding can have an important impact on welfare by changing the geographic-dynamic path of the world economy.
\end{abstract}

\section{Introduction}

An individual's place of residence is essential in determining her productivity, income, and well-being. A person's location, however, is neither a permanent characteristic nor a free choice. People tend to flee undesirable and low productivity areas to go to places that offer better opportunities, but these choices are often hindered by an assortment of restrictions. An obvious example is the effort to stop undocumented migration to Europe, the U.S. and most developed countries. How do these restrictions affect the evolution of the world economy? How do they interact with today's production centers, as well as today's most desirable places to live, to shape the economy of the future? Any attempt to answer these questions requires a theory of development that explicitly takes into account the geography of economic activity and the mobility restrictions and transport costs associated with it. Once we have a basic understanding of the role of geography in development, we can start evaluating the impact of events that change this geography; here, as an application, we study the economic impact of coastal flooding.

Constructing a theory to study the effect of geography on development requires incorporating some well-known forces as well as others that have received, so far, less attention. First, a particular location is unique because

${ }^{*}$ We are grateful to the Gallup Organization and to Angus Deaton for providing us with aggregate national data on wellbeing and on preferred migration destinations from the Gallup World Poll. We also thank Treb Allen, Maya Buchanan, Angus Deaton, Tom Holmes, Chang-Tai Hsieh, Erik Hurst, Steve Redding, Frédéric Robert-Nicoud, Jesse Shapiro, Joe Shapiro, and Rob Townsend for comments and discussions. Desmet: kdesmet@smu.edu. Nagy: dnagy@princeton.edu Rossi-Hansberg: erossi@princeton.edu 
of where it is relative to other locations, which determines its costs of trading goods. Furthermore, each location has particular amenities that determine its desirability as a place to live, and a particular productivity level that determines its desirability as a place to produce and work. This singularity of individual places underscores the importance of bringing the actual geography of the world, as measured by the location of land and water, as well as the distribution of other local spatial and economic characteristics, into the analysis. Second, migration across countries is possible but limited, mostly due to institutional restrictions. National borders restrain mobility well beyond the existing frictions within countries. Third, the distinct levels of labor productivity of locations, which reflect their institutions, infrastructure, education systems, and capital stocks, as well as location-specific technological know-how, evolve over time. Firms can invest in improving this local technology and infrastructure. Not all improvements in technology are local in nature or the result of purposeful investments though. Firms also benefit from the diffusion of the innovations and creativity of others. Fourth, a location's population density affects its productivity, its incentives to innovate, and perhaps most important, its amenities. Large concentrations of people in, for example, urban areas benefit from agglomeration effects but also suffer the undesirable costs from congestion.

Our aim is to study the evolution of the world economy at a rich level of geographic detail (one-degree-by-onedegree resolution) over many years. So our analysis naturally involves many choices and compromises. The basic structure of the model is as follows. Each one-degree-by-one-degree cell of the world contains firms that produce a variety of goods using location-specific technologies that employ labor and a local factor we refer to as land. Firms can trade subject to iceberg transport costs. Each location is endowed with amenities that enhance the quality of life in that cell. The static spatial equilibrium resembles the one proposed in Allen and Arkolakis (2014) with local factors and a trade structure à la Eaton and Kortum (2002). The dynamic model uses this structure and allows firms to invest in improving local technology as in Desmet and Rossi-Hansberg (2014a). These technological innovations determine next period's productivity after taking into account the fact that part of these technologies disseminate over space.

We not only characterize the steady-state distribution of economic activity, but we are also able to compute transitions since, as in Desmet and Rossi-Hansberg (2014a), the innovation decision can be reduced to a simple static problem due to land competition and technological diffusion. Our ability to prove the uniqueness of the equilibrium, to characterize the steady state, to identify the initial distribution of amenities and productivity levels in all locations, and to simulate the model, though novel, is importantly enhanced by the set of results in Zabreyko et al. (1975), first used in spatial models in the related static and single-country model of Allen and Arkolakis (2014). So we owe a substantial debt to this work.

Identifying the reasons why agents locate in a particular place necessarily requires taking a stand on the opportunities they have to move across locations in search of better living and work opportunities. Under the assumption of free mobility, a long tradition in urban and regional economics has identified productivity and amenities across locations using land prices and population counts (see, e.g., Roback, 1982, following the hedonic approach of Rosen, 1979). More recently, another strand of the literature has used income per capita and population counts, together with a spatial equilibrium model, to identify these same local characteristics (see, among many others, Allen and Arkolakis, 2014, Behrens et al., 2013, Desmet and Rossi-Hansberg, 2013, Fajgelbaum and Redding, 2014).

We follow this second strand of the literature, but note that the static nature of these papers yields a decomposition that depends crucially on parameters that are likely to evolve with the level of development of the economy. That is, the parameters used to identify amenities and productivities depend on the particular time 
period in which the exercise was done. As a result, they do not represent stable parameters that one can use to obtain meaningful conclusions in a dynamic context. In fact, in our theoretical framework, the relationship between income, population density, and amenities evolves over time as the economy becomes richer and slowly converges to a balanced growth path. In Appendix A.1 we present some empirical evidence consistent with these theoretical patterns, using data on U.S. counties and zip codes.

Another problematic assumption in this literature is free mobility, particularly given that we are analyzing not only migration across regions within a country but also migration across all countries in the world. While the assumption of free mobility within countries may still be acceptable, it is hard to argue that people anywhere can freely move to the nicest and most productive places on earth. To see this, it suffices to have a casual look at the U.S.-Mexico border, or the restrictions on African immigration in Europe. More important, ignoring mobility restrictions leads to unreasonable conclusions. For example, it would result in an identification of productivities and amenities that makes the Democratic Republic of the Congo one of the nicest places to live on Earth. Although it is hard to take a definitive stand on what characteristics a country's individuals enjoy the most, basic evidence on health, education, governance and institutions suggests that such a conclusion masks the fact that many people in Congo do not choose to live there, but instead are trapped in an undesirable location.

Once we explicitly account for migration restrictions, and therefore utility differences across countries, we get a more nuanced picture. To understand why, note that our theory only identifies amenities relative to utility at each location. Hence, in the absence of mobility restrictions, the large values of amenities relative to utility in Congo would show up as those regions having high amenity levels. But if the Congolese are unable to move to other countries, then the same large values of amenities relative to utility would show up as Congo having a low utility level. Identifying the actual amenity levels therefore involves incorporating more data. To do so, we use survey data on Cantril ladder measures of subjective well-being, as well as actual and desired migration flows from the Gallup World Poll.

The subjective well-being data are an evaluative measure that asks individuals to assess their lives on a ladder scale, from the worst possible to the best possible life they can envision for themselves. Deaton (2008) and Kahneman and Deaton (2010) argue that this measure correlates well with log income and does not exhibit a variety of well-known pathologies that afflict hedonic measures of subjective well-being or happiness. We therefore interpret this evaluative measure as giving us information on the welfare of individuals. Still, we need to convert this ladder with eleven steps into a cardinal measure of the level of utility. To do so, we match the relationship between the ladder measure and log income in the model and in the data. We also present an alternative methodology that uses actual and desired migration flows and yields similar results. Using the cardinal measure of utility together with the amenity to utility ratio, we can recover the actual level of amenities for each cell of the world. As an overidentification check, we find that these estimated amenities correlate well with commonly used exogenous measures of quality of life 1 With these amenities in hand, we are ready to perform a variety of exercises, including counterfactual scenarios with either free mobility or partial removal of observed migration frictions ${ }^{2}$

We calibrate the rest of the model using data on the evolution of output across countries and other information from a variety of sources. We then perform a number of experiments where we simulate the transition of the world economy to its balanced growth path (which takes about 600 years). The parameters we estimate guarantee

\footnotetext{
${ }^{1}$ This suggests that subjective well-being differences capture an essential part of utility differences across countries, although both concepts are unlikely to exactly coincide (see also Glaeser, Gottlieb and Ziv, 2014).

${ }^{2} \mathrm{An}$ alternative approach to incorporate migration restrictions is to use strong distributional assumptions on the location preferences of individuals (as in Kline and Moretti, 2014). See Section 4.5.2 where we incorporate migration data into our analysis using a similar approach.
} 
that the equilibrium is unique and that the economy eventually converges to a balanced growth path in which the geographic distribution of economic activity is constant. The transition to this balanced growth path can, however, take very long. If current migration frictions do not change, it takes about 200 years for the economy to reach its balanced growth path. The protracted length of this transition is the result of the sequential development of clusters due to the complexity of the world's geography. During these 200 years the world experiences large changes. The world's real output growth rate increases progressively, from around $2 \%$ to $3 \%$, while the growth rate of welfare declines from $5 \%$ to slightly above $3 \%$. The correlation between GDP per capita and population density also changes dramatically. The world goes from the current negative correlation of around -0.37 to a high correlation of 0.76 in the balanced growth path 3 That is, in contrast to the world today, where many densely populated areas are poor, in the future the dense regions will be the rich regions.

Relaxing migration restrictions leads to large increases in welfare and productivity at impact. The growth rate of real GDP and welfare also unambiguously increases in the balanced growth path. For example, with the current frictions about $0.74 \%$ of people migrate in a year today, with this percentage converging to zero in the balanced growth path $4^{4}$ If, instead, we drop all restrictions, so there is free mobility, at impact $78.2 \%$ of the population moves across countries and we get welfare gains in the world of $312 \%$ Although this experiment is extreme and we also compute the effects of partial liberalizations, it illustrates the magnitude of the very large gains at stake and it highlights the role of migration policies when thinking about the future of the world economy.

Importantly, differences in migration restrictions put the world on different development paths in which the set of regions that benefit varies dramatically. With the current restrictions, we get a productivity reversal, with many of today's high-density low-productivity regions in sub-Saharan Africa, South Asia and East Asia becoming high-density high-productivity regions, and North America and Europe falling behind in terms of both population and productivity. In contrast, when we relax migration restrictions, Europe and the Eastern areas of the United States remain strong, with certain regions in Brazil and Mexico becoming important clusters of economic activity too.

The driving forces behind these results are complex since the world is so heterogeneous. One of the key determinants of these patterns is the correlation between GDP per capita and population density. As we mentioned above, the correlation is negative and weak today, and our theory predicts that, consistent with the evidence of the U.S. 6 this correlation will become positive and grow substantially over the next six centuries. Two forces drive this result. First, people move to more productive areas, and second, more dense locations become more productive over time since investing in local technologies in dense areas is, in general, more profitable. Migration restrictions shift the balance between these two mechanisms. If migration restrictions are strict, people tend to stay where they are, and today's dense areas, which often coincide with developing countries, become the most developed parts of the world in the future. If, in contrast, migration is free, then people move to the most productive, high-amenity areas. This tends to favor the current developed economies. Liberalizing migration improves welfare so much because it makes the high-productivity regions in the future coincide with the high-amenity locations. So relaxing migration restrictions eliminates the productivity reversal that we observe when migration restrictions are kept

\footnotetext{
${ }^{3}$ The correlation is computed using $1^{\circ} \times 1^{\circ}$ land cells as units.

${ }^{4}$ In the data, the annual migration rate across countries (calculated in an identical way) was $0.6 \%$ in $1961,0.4 \%$ in 1981 , and $0.34 \%$ in 2001. These numbers are a bit lower than in the early periods of our simulation with current migration restrictions, although the model's migration rate converges to zero, with yearly mobility rates declining by about half in the first 30 years.

${ }^{5}$ We use a utilitarian welfare criterion and just calculate the population weighted average utility in the world. Mobility across cells, rather than across countries, when we liberalize is only slightly higher at $79.4 \%$.

${ }^{6}$ See the results for U.S. counties and zip codes in Appendix A.1.
} 
constant.

These results highlight the importance of geography, and the interaction of geography with factor mobility, for the future development path of the world economy. Any policy or shock that affects this geography can have potentially large effects through similar channels. We explore this further by using our framework to evaluate the economic impact of coastal flooding due to climate change. Scientists indicate that sea levels are on the rise as a consequence of the melting of polar ice-sheets and changes in the volume of the water mass due to increases in average world temperatures. These rising sea levels imply that many areas that host millions of residents and workers today will become submerged in water in the future. For example, an increase of 6 meters in the sea level (a fairly extreme scenario) would flood $1 \%$ of land, home to $6.6 \%$ of the world's population.

What is the economic cost of coastal flooding when taking into account the future development path of the world economy? For a 6-meter rise in sea levels, we find welfare losses of around 8.0\%. Two remarks are in order here. First, these are present value welfare losses that take into account future development, not just simple static losses as calculated in most of the related literature (e.g., Dasgupta et al., 2007) 7 Second, welfare losses change very little with migration restrictions.

The finding of welfare losses not depending on mobility is unexpected given the logic in Desmet and RossiHansberg (2014b). In that paper we argue that, in the absence of mobility frictions, local phenomena -like temperature increases due to climate change- should result in small economic losses. Land is an abundant factor in the world; so, as long as adaptation through mobility is an option, the world should be able to escape these negative developments by moving economic activity and residents to areas that are not affected by the phenomena. It is only in the presence of migration restrictions, when adaptation through mobility becomes impossible or costly, that local phenomena can have large effects on welfare.

The findings in our current analysis, though simpler in terms of the link between the economy and climate change, incorporate a much more detailed geography as well as a more detailed description of mobility restrictions. These additions affect the argument above. Free mobility tends to attract more population and development to coastal areas that are in danger of flooding. This implies that when mobility is free and flooding occurs, it affects a greater share of the population and the economy. Hence, although it is easier for people to move, more people suffer the impact of flooding. Moreover, higher mobility does not ensure that people move to the socially optimal places. In the face of flooding, people move to locations where they individually gain most, disregarding both the impact on others and the effect on the future evolution path of the world economy. Although a planner would much prefer to move people to nearby cells in the same country that will not be flooded, in a competitive equilibrium this may not happen. Because of the less detailed geography in Desmet and Rossi-Hansberg (2014b), and the simpler spatial pattern of the climate shock considered there, this issue does not arise. As a result, mobility has small and ambiguous effect on the present value of welfare losses from flooding, although it increases the losses in the long term.

The rest of the paper is organized as follows. Section 2 presents the model and proves the existence and uniqueness of the equilibrium. Section 3 characterizes the balanced growth path of the economy and provides a sufficient condition on the parameters of the model in order for a unique balanced growth path, where the nondegenerate distribution of economic activity is invariant, to exist. Section 4 discusses the calibration of the model, including the inversion to obtain initial productivity and amenity values, as well as the algorithm used to simulate the model. Section 5 presents our numerical findings, which include the results for the benchmark calibration,

\footnotetext{
${ }^{7}$ Section 5.3 includes a more detailed discussion of how our exercise fits into the broader literature on the economic impact of flooding.
} 
different levels of migration frictions, and the change in sea levels. Section 6 concludes. Appendix A presents evidence from U.S. zip codes and counties that shows how the correlation of density and productivity changes with income per capita. It also shows that our estimates of amenities correlate well with exogenous measures of quality of life, and it presents results of the effect of a 1-meter increase in sea level. Appendix B presents the proofs not included in the main text, and Appendix $\mathrm{C}$ discusses the data and their sources in detail. An Online Appendix includes videos with simulations of the world economy for different migration and flooding scenarios.

\section{The Model}

Consider an economy that occupies a two-dimensional surface $S$. A location is a point $r \in S$. Location $r$ has land density $H(r) \geq 0$, where $H(\cdot)$ is an exogenously given continuous function that we normalize so that $\int_{S} H(r) d r=$ 1. There are $C$ countries. Each location belongs to one country, so that countries constitute a partition of $S$ : $\left(S_{1}, \ldots, S_{C}\right)$. The world economy is populated by $\bar{L}$ agents who are endowed with one unit of labor, which they supply inelastically.

\section{$2.1 \quad$ Preferences}

Every period, agents derive utility from local amenities and from consuming a set of differentiated products with CES preferences. An agent's period utility is given by

$$
u_{t}(r)=a_{t}(r)\left[\int_{0}^{1} c_{t}^{\omega}(r)^{\rho} d \omega\right]^{\frac{1}{\rho}}
$$

where $1 /[1-\rho]$ is the elasticity of substitution with $0<\rho<1, a_{t}(r)$ denotes amenities at location $r$ and time $t$, and $c_{t}^{\omega}(r)$ denotes consumption of good $\omega$ at location $r$ and time $t$. Agents discount the future at rate $\beta$ and so the welfare of an individual in the first period is given by $\sum_{t} \beta^{t} u_{t}\left(r_{t}\right)$ where, abusing notation, $r_{t}$ denotes her location choice at $t$. Amenities take the form

$$
a_{t}(r)=\bar{a}(r) \bar{L}_{t}(r)^{-\lambda}
$$

where $\bar{a}(r)$ is an exogenously given positive function, $\bar{L}_{t}(r)$ is population per unit of land at $r$ in period $t$, and $\lambda$ is a fixed parameter where $\lambda \geq 08$ Thus, we allow for congestion externalities in local amenities as a result of high population density, with an elasticity of amenities to population given by $-\lambda$.

We assume that agents cannot write debt contracts with each other. So, as a whole, the residents of a location can save only by investing to enhance the technology to produce in that location in the future, and therefore future local land rents and wages. An agent earns income from work, $w_{t}(r)$, and from the local ownership of land. We assume that local rents are distributed uniformly across a location's residents $9^{9}$ So if $R_{t}(r)$ denotes rents per unit of land, then each agent receives land rent income $R_{t}(r) / \bar{L}_{t}(r)$. Total income of an agent in location $r$ at time $t$ is therefore $w_{t}(r)+R_{t}(r) / \bar{L}_{t}(r)$. Let $P_{t}(r)$ denote the ideal price index at location $r$ in period $t$, where

\footnotetext{
${ }^{8}$ This is consistent with the positive value of $\lambda$ we find in our estimation, although the theory could in principle allow for a negative number, in which case there would be positive amenities from agglomeration.

${ }^{9}$ See Caliendo et al. (2014) for alternative assumptions on land ownership and their implications.
} 


$$
\begin{aligned}
& P_{t}(r)=\left[\int_{0}^{1} p_{t}^{\omega}(r)^{-\frac{\rho}{1-\rho}} d \omega\right]^{-\frac{1-\rho}{\rho}} \text {. Then } \\
& \qquad u_{t}(r)=a_{t}(r) y_{t}(r)=a_{t}(r) \frac{w_{t}(r)+R_{t}(r) / \bar{L}_{t}(r)}{P_{t}(r)},
\end{aligned}
$$

where $y_{t}(r)$ denotes real income.

Agents are freely mobile within countries, but we allow for migration restrictions that create differences in utility levels across countries. In particular, agents can migrate freely up to the point at which the relative utility levels of countries are left unchanged over time. Beyond this point, migration is prohibited. That is, we model migration as a quota that adjusts to keep relative utility levels constant ${ }^{10}$ As an example, consider a receiving country and a sending country, and suppose the utility in the receiving country goes up. In that case, the receiving country relaxes the quota, more migrants enter, and the utility of the receiving country relative to that of the sending country returns to its original level. If relative utility levels do not change over time, the equilibrium utility of an agent in location $r$ in country $c$ can then be written as $\bar{u}_{t}^{W} \bar{u}(c)$, where $\bar{u}_{t}^{W}$ is common across countries but changing over time, and $\bar{u}(c)$ is country-specific but constant over time (and averages to one). Hence, in the special case of free mobility, utility levels equalize across countries and so $\bar{u}(c)=1$.

\subsection{Technology}

Firms produce a good $\omega \in[0,1]$ using land and labor. A firm using $L_{t}^{\omega}(r)$ production workers per unit of land at location $r$ at time $t$ produces

$$
q_{t}^{\omega}(r)=\phi_{t}^{\omega}(r)^{\gamma_{1}} z_{t}^{\omega}(r) L_{t}^{\omega}(r)^{\mu}
$$

units of good $\omega$ per unit of land, where $\gamma_{1}, \mu \in(0,1]$. A firm's productivity is determined by its decision on the quality of its technology - what we call an innovation $\phi_{t}^{\omega}(r)$ - and an exogenous local and good-specific productivity shifter $z_{t}^{\omega}(r)$. To use an innovation $\phi_{t}^{\omega}(r)$, the firm has to employ $\nu \phi_{t}^{\omega}(r)^{\xi}$ additional units of labor per unit of land, where $\xi>\gamma_{1} /[1-\mu]$. The exogenous productivity shifter $z_{t}^{\omega}(r)$ is the realization of a random variable that is i.i.d. across goods and time periods. It is drawn from a Fréchet distribution with c.d.f.

$$
F(z, r)=e^{-T_{t}(r) z^{-\theta}}
$$

where $T_{t}(r)=\tau_{t}(r) \bar{L}_{t}(r)^{\alpha}$, and $\alpha \geq 0, \theta>0$ are exogenously given. The value of $\tau_{t}(r)$ is determined by an endogenous dynamic process that depends on past innovation decisions in this location and potentially in others, $\phi^{\omega} \cdot(\cdot)$.

We assume that the initial productivity $\tau_{0}(\cdot)$ is an exogenously given positive continuous function. Conditional on the spatial distribution of productivity in period $t-1, \tau_{t-1}(\cdot)$, the productivity at location $r$ in period $t$ is given by

$$
\tau_{t}(r)=\phi_{t-1}(r)^{\theta \gamma_{1}}\left[\int_{S} \eta \tau_{t-1}(s) d s\right]^{1-\gamma_{2}} \tau_{t-1}(r)^{\gamma_{2}}
$$

\footnotetext{
${ }^{10}$ Alternatively, we could assume that migrants face a utility cost that is proportional to welfare in the destination country. These iceberg utility costs would be fixed, so as to leave relative utility levels across countries constant. The equilibrium allocation is not affected by whether we use a cost or a quota, although the individual utility gains from migration, of course, are. We believe it is more appealing to assume that migrants gain in relative utility terms and so use the quota interpretation in our welfare gains calculations throughout.
} 
where $\eta$ is a constant such that $\int_{S} \eta d r=1$ and $\gamma_{1}, \gamma_{2} \in[0,1]$. If $\gamma_{2}=1$ and population density is constant over time, this implies that the mean of $z_{t}^{\omega}(r)$ is $\phi_{t-1}(r)^{\gamma_{1}}$ times the mean of $z_{t-1}^{\omega}(r){ }^{11}$ That is, the distribution of productivity draws is shifted up by past innovations, but with decreasing returns if $\gamma_{1}<1$. If $\gamma_{2}<1$, the dynamic evolution of a location's technology also depends on the aggregate level of technology, $\int_{S} \eta \tau_{t}(s) d s$.

Later we will see that assuming $\gamma_{1}, \gamma_{2} \in(0,1)$ helps with the convergence properties of the model since we can have local decreasing returns, but economy-wide linear technological progress. If $\gamma_{2}=1$, the evolution of local technology is independent of aggregate technology and, as we will show below, in a balanced growth path in which the economy is growing, economic activity will generally end up concentrating in a unique point. In contrast, if $\gamma_{1}=\gamma_{2}=0$, only the aggregate evolution of technology matters, there are no incentives to innovate, and the economy stagnates. Therefore, in the calibration we will necessary obtain values of $\gamma_{1}$ and $\gamma_{2}$ strictly in between 0 and 1. We find that the distribution of growth rates in the world implies a high $\gamma_{2}=0.993$.

Across locations $z_{t}^{\omega}(r)$ is assumed to be spatially correlated. In particular, we assume that the productivity draws for a particular variety in a given period are perfectly correlated for neighboring locations as the distance between them goes to zero. We also assume that at large enough distances the draws are independent. This implies that the law of large numbers still applies in the sense that a particular productivity draw has no aggregate effects. Formally, let $\zeta_{t}^{\omega}(r, s)$ denote the correlation in the draws $z_{t}^{\omega}(r)$ and $z_{t}^{\omega}(s)$ and let $\delta(r, s)$ denote the distance between $r$ and $s$. We assume that there exists a continuous function $s(d)$ where $\delta(r, s(d))=d$ such that $\lim _{d \rightarrow 0} \zeta_{t}^{\omega}(r, s(d)) \rightarrow 1$. Furthermore, $\zeta_{t}^{\omega}(r, s)=0$ for $\delta(r, s)$ large enough. One easy example is having land divided into regions of positive area, where $\zeta_{t}^{\omega}(r, s)=1$ within a region and $\zeta_{t}^{\omega}(r, s)=0$ otherwise.

Since firm profits are linear in land, for any small interval with positive measure there is a continuum of firms that compete in prices (à la Bertrand). Note that the spatial correlation of the productivity draws, as well as the continuity of amenities and transport costs in space, implies that the factor prices and transport costs faced by these firms will be similar in a small interval. Hence, Bertrand competition implies that their pricing will be similar as well. As the size of the interval goes to zero, these price differences converge to zero, leading to an economy in which firms face perfect local competition.

Local competition implies that firms will bid for land up to the point at which they obtain zero profits after covering their investment in technology $w_{t}(r) \nu \phi_{t}^{\omega}(r)^{\xi}$. So even though this investment in technology affects productivity in the future through equation (4), the investment decision at any given point can safely disregard this dependence given the absence of future profits regardless of the level of investment. This implies that the solution to the dynamic innovation decision problem is identical to a sequence of static innovation decisions that maximize static profits. Firms innovate in order to maximize their bid for land, win the land auction, and produce. This decision affects the economy in the future, but not the future profits of the firm, which are always zero. The implication, as discussed in detail in Desmet and Rossi-Hansberg (2014a), is that we only need to solve the static optimization problem of the firm and can disregard equation (4) in the firm's problem.

Therefore, after learning their common local productivity draw, $z_{t}^{\omega}(r)$, a potential firm at $r$ maximizes its

${ }^{11}$ To obtain the mean of the standard Fréchet distribution $F(z)=e^{-T_{t} z^{-\theta}}$, first write down the density function $f(z)=$ $\theta T_{t} z^{-\theta-1} e^{-T_{t} z^{-\theta}}$. The mean is then $\int_{0}^{\infty} z f(z) d z=\int_{0}^{\infty} \theta T_{t} z^{-\theta} e^{-T_{t} z^{-\theta}} d z$. Remember that $\Gamma(\alpha)=\int_{0}^{\infty} y^{\alpha-1} e^{-y} d y$. Redefine $T_{t} z^{-\theta}=y$, so that $d y=-\theta T_{t} z^{-\theta-1} d z$. Substitute this into the previous expression, so that $\int_{\infty}^{0} \frac{T_{t} \theta z^{-\theta}}{-\theta T_{t} z^{-\theta-1}} e^{-y} d y=$ $\int_{\infty}^{0}-z e^{-y} d y=T_{t}^{\frac{1}{\theta}} \int_{0}^{\infty} y^{-\frac{1}{\theta}} e^{-y} d y=T_{t}^{\frac{1}{\theta}} \Gamma\left(\frac{\theta-1}{\theta}\right)$, where $T_{t}=\tau_{t} L_{t}^{\alpha}$. If $\gamma_{2}=1$, we have $\tau_{t}=\phi_{t-1}^{\theta \gamma_{1}} \tau_{t-1}$. Assuming the labor force does not change over time, we can write $\frac{T_{t}}{T_{t-1}}=\frac{\tau_{t}}{\tau_{t-1}}=\phi_{t-1}^{\theta \gamma_{1}}$, so that $T_{t}=\phi_{t-1}^{\theta \gamma_{1}} T_{t-1}$. Hence, the expectation is given by $E\left(z_{t}\right)=T_{t}^{\frac{1}{\theta}} \Gamma\left(\frac{\theta-1}{\theta}\right)=\phi_{t-1}^{\gamma_{1}} T_{t-1}^{\frac{1}{\theta}} \Gamma\left(\frac{\theta-1}{\theta}\right)=\phi_{t-1}^{\gamma_{1}} E\left(z_{t-1}\right)$. 
current profits per unit of land by choosing how much labor to employ and how much to innovate,

$$
\max _{L_{t}^{\omega}(r), \phi_{t}^{\omega}(r)} p_{t}^{\omega}(r, r) \phi_{t}^{\omega}(r)^{\gamma_{1}} z_{t}^{\omega}(r) L_{t}^{\omega}(r)^{\mu}-w_{t}(r) L_{t}^{\omega}(r)-w_{t}(r) \nu \phi_{t}^{\omega}(r)^{\xi}-R_{t}(r)
$$

where $p_{t}^{\omega}(r, r)$ is the price charged by the firm of a good sold at $r$, which is equivalent to the price the firm charges in another location net of transport costs. The two first-order conditions are

$$
\mu p_{t}^{\omega}(r, r) \phi_{t}^{\omega}(r)^{\gamma_{1}} z_{t}^{\omega}(r) L_{t}^{\omega}(r)^{\mu-1}=w_{t}(r)
$$

and

$$
\gamma_{1} p_{t}^{\omega}(r, r) \phi_{t}^{\omega}(r)^{\gamma_{1}-1} z_{t}^{\omega}(r) L_{t}^{\omega}(r)^{\mu}=\xi w_{t}(r) \nu \phi_{t}^{\omega}(r)^{\xi-1}
$$

So a firm's bid rent per unit of land is given by

$$
R_{t}(r)=p_{t}^{\omega}(r, r) \phi_{t}^{\omega}(r)^{\gamma_{1}} z_{t}^{\omega}(r) L_{t}^{\omega}(r)^{\mu}-w_{t}(r) L_{t}^{\omega}(r)-w_{t}(r) \nu \phi_{t}^{\omega}(r)^{\xi},
$$

which ensures all firms make zero profits. Using (5) and (6) gives

$$
\frac{L_{t}^{\omega}(r)}{\mu}=\frac{\xi \nu \phi_{t}^{\omega}(r)^{\xi}}{\gamma_{1}}
$$

Then, total employment at $r$ for variety $\omega, \bar{L}_{t}^{\omega}(r)$, is the the sum of production workers, $L_{t}^{\omega}(r)$, and innovation workers, $\nu \phi_{t}^{\omega}(r)^{\xi}$, so

$$
\bar{L}_{t}^{\omega}(r)=L_{t}^{\omega}(r)+\nu \phi_{t}^{\omega}(r)^{\xi}=\frac{L_{t}^{\omega}(r)}{\mu}\left[\mu+\frac{\gamma_{1}}{\xi}\right]
$$

Note also that

$$
R_{t}(r)=\left[\frac{\xi(1-\mu)}{\gamma_{1}}-1\right] w_{t}(r) \nu \phi_{t}^{\omega}(r)^{\xi}
$$

so bid rents are proportional and increasing in a firm's investment in technology, $w_{t}(r) \nu \phi_{t}^{\omega}(r)^{\xi}$, as we argued above.

In equilibrium firms take the bids for land by others, and therefore the equilibrium land rent, as given and produce in a location if their land bid is greater than or equal to the equilibrium land rent. Hence, in equilibrium, in a given location, the amount of workers hired per unit of land and the amount of innovation done per unit of land are identical across goods. We state this formally in the following result.

Lemma 1 The decision of how much to innovate, $\phi_{t}^{\omega}(r)$, and how many workers to hire per unit of land, $\bar{L}_{t}^{\omega}(r)$, is independent of the local idiosyncratic productivity draws, $z_{t}^{\omega}(r)$, and so are identical across goods $\omega$.

Proof. Since in equilibrium $R_{t}(r)$ is taken as given by firms producing at $r$, the proof is immediate by inspecting (8), (9), and (10).

This will greatly simplify the analysis, as it will provide us with a relation between $p_{t}^{\omega}(r, r)$ and $z_{t}^{\omega}(r)$ similar to the one in Eaton and Kortum (2002) in spite of firms being able to innovate. Combining the expressions above 
yields an expression for the price of a good produced at $r$ and sold at $r$ :

$$
p_{t}^{\omega}(r, r)=\left[\frac{1}{\mu}\right]^{\mu}\left[\frac{\nu \xi}{\gamma_{1}}\right]^{1-\mu}\left[\frac{\gamma_{1} R_{t}(r)}{w_{t}(r) \nu\left(\xi(1-\mu)-\gamma_{1}\right)}\right]^{(1-\mu)-\frac{\gamma_{1}}{\xi}} \frac{w_{t}(r)}{z_{t}^{\omega}(r)} .
$$

To facilitate subsequent notation, we rewrite the above expression as

$$
p_{t}^{\omega}(r, r)=\frac{m c_{t}(r)}{z_{t}^{\omega}(r)}
$$

where $m c_{t}(r)$ denotes the input cost in location $r$ at time $t$, namely,

$$
m c_{t}(r) \equiv\left[\frac{1}{\mu}\right]^{\mu}\left[\frac{\nu \xi}{\gamma_{1}}\right]^{1-\mu}\left[\frac{\gamma_{1} R_{t}(r)}{w_{t}(r) \nu\left(\xi(1-\mu)-\gamma_{1}\right)}\right]^{(1-\mu)-\frac{\gamma_{1}}{\xi}} w_{t}(r)
$$

It is key to understand that from the point of view of the individual firm, this input cost $m c_{t}(r)$ is given. As a result, expression 12 describes a straightforward relation between the productivity draw $z_{t}^{\omega}(r)$ and the price $p_{t}^{\omega}(r, r)$. As in Eaton and Kortum (2002), this is what allows us in the next subsection to derive probabilistic expressions on a location's price distribution, its probability of exporting to other locations, and its share of exports.

\subsection{Prices, Export Probabilities and Export Shares}

Let $\varsigma(s, r) \geq 1$ denote the iceberg cost of transporting a good from $r$ to $s$. Assume $\varsigma(\cdot, \cdot)$ is a continuous function. Therefore, the price of a good $\omega$, produced in $r$ and sold in $s$, will be

$$
p_{t}^{\omega}(s, r)=p_{t}^{\omega}(r, r) \varsigma(s, r)=\frac{m c_{t}(r) \varsigma(s, r)}{z_{t}^{\omega}(r)} .
$$

As we derive formally in Appendix B, the probability that a given good produced in an area $r$ is bought in $s$ is given by

$$
\pi_{t}(s, r)=\frac{T_{t}(r)\left[m c_{t}(r) \varsigma(r, s)\right]^{-\theta}}{\int_{S} T_{t}(u)\left[m c_{t}(u) \varsigma(u, s)\right]^{-\theta} d u} \text { all } r, s \in S .
$$

The price index of a location $s$, as we also show in Appendix $\mathrm{B}$, is then given by

$$
P_{t}(s)=\Gamma\left(\frac{-\rho}{(1-\rho) \theta}+1\right)^{-\frac{1-\rho}{\rho}}\left[\int_{S} T_{t}(u)\left[m c_{t}(u) \varsigma(s, u)\right]^{-\theta} d u\right]^{\frac{-1}{\theta}}
$$

where $\Gamma$ denotes the gamma function.

\subsection{Trade Balance}

We impose trade balance location by location since there is no mechanism for borrowing from or lending to other agents. Market clearing requires total revenue in location $r$ to be equal to total expenditure on goods from $r$. Total revenue at $r$ is $w_{t}(r) H(r)\left[L_{t}(r)+\nu \phi_{t}(r)^{\xi}\right]+H(r) R_{t}(r)=\frac{1}{\mu} w_{t}(r) H(r) L_{t}(r)$, where the last equality comes from (8), (9) and (10). As in Eaton and Kortum (2002), the fraction of goods that location $s$ buys from $r, \pi_{t}(s, r)$, 
is equal to the fraction of expenditure on goods from $r$, so that the trade balance condition can be written as

$$
w_{t}(r) H(r) \bar{L}_{t}(r)=\int_{S} \pi_{t}(s, r) w_{t}(s) H(s) \bar{L}_{t}(s) d s \text { all } r \in S .
$$

where the superscript $\omega$ can be dropped because the number of workers does not depend on the good a firm produces, and $L$ can be replaced by $\bar{L}$ because the proportion of total workers to production workers is constant across locations.

\subsection{Equilibrium}

We define a dynamic competitive equilibrium as follows:

Definition 1 Given a set of countries, $S_{c} \subset S$, and their relative utility levels, $\bar{u}(c)$, initial technology, amenity and land functions $\left(\tau_{0}, \bar{a}, H\right): S \rightarrow \mathbb{R}$, a competitive equilibrium with potentially restricted mobility is a set of functions $\left(\bar{L}_{t}, \phi_{t}, R_{t}, w_{t}, P_{t}, \tau_{t}, T_{t}\right): S \rightarrow \mathbb{R}$ for all $t=1, \ldots$, as well as a pair of functions $\left(p_{t}^{\cdot}, c_{t}^{\cdot}\right):[0,1] \times S \rightarrow \mathbb{R}$ and a scalar $\bar{u}_{t}^{W}$, for all $t=1, \ldots$, such that:

1. Firms optimize and markets clear. Namely, (5), (6) and (9) hold.

2. The share of income of location s spent on goods of location $r$ is given, for all t, by 13) and 15.)

3. Trade balance implies that (17) holds for all $r \in S$ and all $t$.

4. Land markets are in equilibrium, so land is assigned to the highest bidder. Thus,

$$
R_{t}(r)=\left[\frac{\xi-\mu \xi-\gamma_{1}}{\mu \xi+\gamma_{1}}\right] w_{t}(r) \bar{L}_{t}(r)
$$

5. The utility of location $r$ in country $c$ and time $t$ is given by

$$
\bar{u}_{t}^{W} \bar{u}(c)=a_{t}(r) \frac{w_{t}(r)+R_{t}(r) / \bar{L}_{t}(r)}{P_{t}(r)}=\bar{a}(r) \bar{L}_{t}(r)^{-\lambda} \frac{\xi}{\mu \xi+\gamma_{1}} \frac{w_{t}(r)}{P_{t}(r)} \text { for all } r \in S
$$

where the price index $P_{t}(\cdot)$ is given by $[16$.

6. Labor markets clear so

$$
\int_{S} H(r) \bar{L}_{t}(r) d r=\bar{L}
$$

7. Technology evolves according to

$$
\tau_{t}(r)=\phi_{t-1}(r)^{\theta \gamma_{1}}\left[\int_{S} \eta \tau_{t-1}(s) d s\right]^{1-\gamma_{2}} \tau_{t-1}(r)^{\gamma_{2}} .
$$

We can manipulate the system of equations that defines an equilibrium and, ultimately, reduce it to a system of equations that determines wages and employment levels in all locations. In a given period, the following lemma characterizes the relationship between labor density and wages conditional on $\bar{a}(\cdot), \tau_{t}(\cdot), \varsigma(\cdot, \cdot), \bar{u}_{c}, \bar{u}_{t}^{W}$, and parameter values. Appendix B presents all proofs not included in the main text. 
Lemma 2 Given $\tau_{t}(\cdot), \bar{u}_{t}^{W}$, and $\bar{u}(c)$, if trade costs are symmetric so that $\varsigma(r, s)=\varsigma(s, r)$, all $s, r \in S$, the equilibrium wage schedule $w_{t}(\cdot)$ and population density schedule $L_{t}(\cdot)$ satisfies

$$
w_{t}(r)=\bar{w}\left[\frac{\bar{a}(r)}{\bar{u}(c)}\right]^{-\frac{\theta}{1+2 \theta}} \tau_{t}(r)^{\frac{1}{1+2 \theta}} H(r)^{-\frac{1}{1+2 \theta}} \bar{L}_{t}(r)^{\frac{\alpha-1+\left[\lambda+\frac{\gamma_{1}}{\xi}-[1-\mu]\right] \theta}{1+2 \theta}}
$$

and

$$
\begin{aligned}
& {\left[\frac{\bar{a}(r)}{\bar{u}(c)}\right]^{-\frac{\theta(1+\theta)}{1+2 \theta}} \tau_{t}(r)^{-\frac{\theta}{1+2 \theta}} H(r)^{\frac{\theta}{1+2 \theta}} \bar{L}_{t}(r)^{\lambda \theta-\frac{\theta}{1+2 \theta}\left[\alpha-1+\left[\lambda+\frac{\gamma_{1}}{\xi}-[1-\mu]\right] \theta\right]} } \\
= & {\left[\bar{u}_{t}^{W}\right]^{-\theta} \kappa_{1} \sum_{d=1}^{C} \int_{S_{d}}\left[\frac{\bar{a}(s)}{\bar{u}(d)}\right]^{\frac{\theta^{2}}{1+2 \theta}} \tau_{t}(s)^{\frac{1+\theta}{1+2 \theta}} H(s)^{\frac{\theta}{1+2 \theta}} \varsigma(r, s)^{-\theta} \bar{L}_{t}(s)^{1-\lambda \theta+\frac{1+\theta}{1+2 \theta}\left[\alpha-1+\left[\lambda+\frac{\gamma_{1}}{\xi}-[1-\mu]\right] \theta\right]} d s . }
\end{aligned}
$$

where $C$ denotes the number of countries, such that $\cup_{C} S_{c}=S, r \in S_{c}$, and $\kappa_{1}$ is a constant.

Following Allen and Arkolakis (2014) we can then use Theorem 2.19 in Zabreyko et al. (1975) to prove that, if

$$
\frac{\alpha}{\theta}+\frac{\gamma_{1}}{\xi} \leq \lambda+1-\mu
$$

there exists a unique positive $\bar{L}_{t}(\cdot)$ that solves 21). Given an equilibrium $\bar{L}_{t}(\cdot)$, all other variables are determined by the equations above, and $\tau_{t+1}$ is uniquely determined by $(19)$. This immediately guarantees the existence and uniqueness of an equilibrium. We summarize this result in the following Lemma.

Lemma 3 An equilibrium of this economy exists and is unique if $\alpha / \theta+\gamma_{1} / \xi \leq \lambda+1-\mu$.

Proof. Theorem 2.19 in Zabreyko, et al. (1975) guarantees that there exists a unique solution to equation (21) as long as the ratio of exponents to $\bar{L}_{t}(r)$ on the RHS and LHS of 21 is weakly smaller than one, namely,

$$
1-\lambda \theta+\frac{1+\theta}{1+2 \theta}\left[\alpha-1+\left[\lambda+\frac{\gamma_{1}}{\xi}-[1-\mu]\right] \theta\right] \leq \lambda \theta-\frac{\theta}{1+2 \theta}\left[\alpha-1+\left[\lambda+\frac{\gamma_{1}}{\xi}-[1-\mu]\right] \theta\right],
$$

which, after simplifying, leads to the condition in the statement of the Lemma.

\section{The Balanced Growth Path}

In a balanced growth path (BGP) of the economy, if one exists, all regions grow at the same rate. A BGP might not exist; instead, all economic activity might eventually concentrate in one point, or the economy may cycle without reaching a BGP. Given the evolution of technology in $(19)$, the growth rate of $\tau_{t}(r)$ is given by

$$
\frac{\tau_{t+1}(r)}{\tau_{t}(r)}=\phi_{t}(r)^{\theta \gamma_{1}}\left[\int_{S} \eta \frac{\tau_{t}(s)}{\tau_{t}(r)} d s\right]^{1-\gamma_{2}} \text {. }
$$

Hence, in a BGP in which technology growth rates are constant, so $\frac{\tau_{t+1}(r)}{\tau_{t}(r)}$ is constant over time and space and $\frac{\tau_{t}(s)}{\tau_{t}(r)}$ is constant over time, the investment decision will be constant but different across locations. Divide both 
sides of the equation by the corresponding equation for location $s$, and rearrange to get

$$
\frac{\tau_{t}(s)}{\tau_{t}(r)}=\left[\frac{\phi(s)}{\phi(r)}\right]^{\frac{\theta \gamma_{1}}{1-\gamma_{2}}}=\left[\frac{\bar{L}(s)}{\bar{L}(r)}\right]^{\frac{\theta \gamma_{1}}{\left[1-\gamma_{2}\right] \xi}}
$$

where the second equality follows from (8) and where we drop the time subscript to indicate that we refer to a variable that remains constant in the BGP. We can then use Lemma 2 and the labor market clearing condition to derive an equation that determines the spatial distribution of productivity $\tau_{t}(r)$ on the balanced growth path. According to Theorem 2.19 in Zabreyko et al. (1975) a unique positive solution to that equation exists if

$$
\frac{\alpha}{\theta}+\frac{\gamma_{1}}{\xi}+\frac{\gamma_{1}}{\left[1-\gamma_{2}\right] \xi} \leq \lambda+1-\mu
$$

This condition is strictly stronger than the condition that guarantees the existence and uniqueness of an equilibrium in Lemma 3 since it includes an extra positive term on the left-hand-side. The condition is also intuitive. On the left hand side we have the two static agglomeration effects: agglomeration externalities $(\alpha / \theta)$ and improvements in local technology for today's production $\left(\gamma_{1} / \xi\right)$. The third term, which appears in the condition for the balanced growth path only, is related to the dynamic agglomeration effect from local investments in technology as well as diffusion $\left(\gamma_{1} /\left[1-\gamma_{2}\right] \xi\right)$. In fact, without diffusion, when $1-\gamma_{2}=0$, condition 22 is never satisfied and there is no BGP with a non-degenerate distribution of employment. On the right-hand-side of condition (22) we have the parameters governing the two congestion forces, namely, congestion through lower amenities $(\lambda)$ and congestion through lower land per worker $(1-\mu)$. So condition 22 simply says that in order for the economy to have a unique BGP, the congestion forces have to be large enough relative to the agglomeration forces. Similarly, the condition in Lemma 3 says that congestion forces have to be strong enough relative to static agglomeration forces in order for an equilibrium to exist. The difference is that an equilibrium can exist even if condition 22 is violated since the dynamic agglomeration effect leads economic activity to progressively concentrate in an area of measure zero.

We summarize the result in the following Lemma.

Lemma 4 If $\alpha / \theta+\gamma_{1} / \xi+\gamma_{1} /\left[\left[1-\gamma_{2}\right] \xi\right] \leq \lambda+[1-\mu]$, then there exists a unique balanced growth path with a constant distribution of employment densities $\bar{L}(\cdot)$ and innovation $\phi(\cdot)$. In the BGP $\tau_{t}(r)$ grows at a constant rate for all $r \in S$.

The condition that determines $\tau_{t}(r)$ in the BGP (which we write explicitly in the proof of Lemma 4 ) guarantees that in the BGP welfare grows according to

$$
\frac{\bar{u}_{t+1}^{W}}{\bar{u}_{t}^{W}}=\left[\frac{\tau_{t+1}(r)}{\tau_{t}(r)}\right]^{\frac{1}{\theta}} .
$$

We can then use the equations above to show that the growth rate of world utility (or the growth rate of real output) is a function of the distribution of employment in the balanced growth path.

Lemma 5 In a balanced growth path, under the conditions of Lemma 4, aggregate welfare and aggregate real 
consumption grow according to

$$
\frac{\bar{u}_{t+1}^{W}}{\bar{u}_{t}^{W}}=\left[\frac{\int_{0}^{1} c_{t+1}^{\omega}(r)^{\rho} d \omega}{\int_{0}^{1} c_{t}^{\omega}(r)^{\rho} d \omega}\right]^{\frac{1}{\rho}}=\eta^{\frac{1-\gamma_{2}}{\theta}}\left[\frac{\gamma_{1} / \nu}{\gamma_{1}+\mu \xi}\right]^{\frac{\gamma_{1}}{\xi}}\left[\int_{S} \bar{L}(s)^{\frac{\theta \gamma_{1}}{\left[1-\gamma_{2}\right] \xi}} d s\right]^{\frac{1-\gamma_{2}}{\theta}} .
$$

Hence welfare and aggregate real output growth depend on population size and its distribution in space.

\section{Calibration and Simulation of the Model}

In order to compute the equilibrium of the model we need values for the nine parameters used in the equations above, in addition to values for initial productivities and amenities for all locations. We also need values for transportation costs between any two cells. If we are interested in scenarios where migratory restrictions are kept as in the world today, we only need to recover amenities relative to utility levels, since equations (21) and (20) only depend on $\bar{a}(r) / \bar{u}(c)$. However, when we consider scenarios with counterfactual mobility frictions, we need to identify amenities and utility levels separately. In that case we also need values for the relative utility levels for all locations. Once we have numbers for all of these variables and parameters, we can compute the model with a simple iterative algorithm.

Table 1 lists the parameter values and gives a brief explanation of how they are assigned. When assigning parameter values, we assume a model period to be one year, so we set $\beta=0.95$. We base some of the parameter values on those in the existing literature. We estimate other parameter values using our model. In what follows we start by briefly discussing some of the parameter values that come from the literature, and then provide a detailed discussion of how we estimate the remaining parameters.

The elasticity of substitution, $1 /(1-\rho)$, is set to 4 , similar to the 3.8 estimated in Bernard et al. (2003). We choose a trade elasticity, $\theta$, equal to 6.5 , somewhere in the middle between the 8.3 value estimated by Eaton and Kortum (2002) and the 4.6 value estimated by Simonovska and Waugh (2014). The labor share in production, $\mu$, is set to 0.8. While higher than the standard labor share, this parameter should be interpreted as the non-land share. Desmet and Rappaport (2014) find a land share of 0.1 when accounting for the land used in both production and housing. Taking a broader view of land by including structures, this share increases to around 0.2, based on a structures share slightly above 0.1, as calibrated by Greenwood, Hercowitz and Krusell (1997). We therefore take the non-land share to be 0.8 but have checked that our main results are robust to alternative values for this parameter.

\subsection{Amenity Parameter}

The theory assumes that a location's amenities decrease with its population. As given by $a(r)=\bar{a}(r) \bar{L}(r)^{-\lambda}$, the parameter $\lambda$ represents the elasticity of amenities to population. Taking logs gives us the following equation:

$$
\log (a(r))=E(\log (\bar{a}(r)))-\lambda \log \bar{L}(r)+\varepsilon(r)
$$

where $E(\log (\bar{a}(r)))$ is the mean of $\log (\bar{a}(r))$ across locations, and $\varepsilon(r)$ is the location-specific deviation of $\log (\bar{a}(r))$ from the mean. Assuming that amenities are log-normally distributed across locations, we use data from Desmet and Rossi-Hansberg (2013) on amenities and population for 192 metropolitan statistical areas (MSAs) in 
Table 1: Parameter Values

\begin{tabular}{ll}
\hline \hline Parameter & Target/Comment \\
\hline 1. Preferences: $\sum_{t} \beta^{t} u_{t}\left(r_{t}\right)$ where $u_{t}(r)=\bar{a}(r) \overline{L_{t}}(r)^{-\lambda}(r)\left[\int_{0}^{1} c_{t}^{\omega}(r)^{\rho} d \omega\right]^{1 / \rho}$ and $e^{\psi \tilde{u}_{i}(r)}$ \\
$\beta=0.95$ & Discount factor \\
$\rho=0.75$ & Elasticity of substitution of 4 (Bernard et al., 2003) \\
$\lambda=0.32$ & Relation between amenities and population \\
$\psi=1.8$ & Relation between actual migration, desired migration and subjective well-being \\
\hline $\mathbf{2 .}$ Technology: $q_{t}^{\omega}(r)=\phi_{t}^{\omega}(r)^{\gamma_{1}} z_{t}^{\omega}(r) L_{t}^{\omega}(r)^{\mu}, F(z, r)=e^{-T_{t}^{\omega}(r) z^{-\theta}}$ and $T_{t}^{\omega}(r)=\tau_{t}(r) \overline{L_{t}}(r)^{\alpha}$ \\
$\alpha=0.06$ & Elasticity of productivity to density $($ Ciccone and Hall, 1996) \\
$\theta=6.5$ & Trade elasticity (Eaton and Kortum, 2002; Simonovska and Waugh, 2014) \\
$\mu=0.8$ & Labor or non-land share in production \\
& (Greenwood, Hercowitz and Krusell, 1997; Desmet and Rappaport, 2014) \\
$\gamma_{1}=0.319$ & Relation between population distribution and growth \\
\hline 3. Evolution of productivity: $\tau_{t}(r)=\phi_{t-1}(r)^{\theta \gamma_{1}}\left[\int_{S} \eta \tau_{t-1}(s) d s\right]^{1-\gamma_{2}} \tau_{t-1}(r)^{\gamma_{2}}$ and $\psi(\phi)=\nu \phi^{\xi}$ \\
$\gamma_{2}=0.993$ & Relation between population distribution and growth \\
$\xi=125$ & Desmet and Rossi-Hansberg $(2014 \mathrm{a})$ \\
$\nu=0.2$ & Initial world growth rate of real GDP of $2 \%$ \\
\hline 4. Trade Costs & \\
$\sigma_{\text {rail }}=0.1434$ & \\
$\sigma_{\text {no_rail }}=0.4302$ & \\
$\sigma_{\text {major_road }}=0.5636$ & \\
$\sigma_{\text {other_road }}=1.1272$ & Allen and Arkolakis $(2014)$ \\
$\sigma_{\text {no_road }}=1.9726$ & \\
$\sigma_{\text {water }}=0.0779$ & \\
$\sigma_{\text {no_water }}=0.779$ & \\
\hline \hline
\end{tabular}

the United States to estimate equation (24). One remaining issue is that population does not only affect amenities, but amenities also affect population. To deal with this problem of reverse causality, we use an MSA's exogenous productivity level as an instrument for its population. Desmet and Rossi-Hansberg (2013) provide estimates for the exogenous productivity of MSAs which they define as the productivity that is not due to agglomeration economies. When using this as an instrument, the identifying assumption is that a location's exogenous productivity does not affect its amenities directly, but only indirectly through the level of its population. This is consistent with the assumptions of our model. Estimating (24) by 2SLS yields a value of $\lambda=0.32$, which is what we report in Table 1 .

\subsection{Technology Parameters}

Our starting point is the economy's balanced utility growth equation (23). To exploit the cross-country variation in growth rates in the data, assume that all countries are in a balanced growth path, but their growth rates may differ ${ }^{12}$ In that case we can rewrite $[23$, after taking logs and discretizing space into cells, as

\footnotetext{
${ }^{12}$ Essentially, we are assuming that the relative distribution of population within countries has converged to what would be observed in a balanced growth path, although international migration flows may still change the relative distribution of population across countries. As a result, growth rates may differ across countries, although each country is characterized by (23). As an alternative to this simplifying assumption, we could use the whole structure of the model calibrated for 1990, and then estimate the parameters that make the simulated model match the observed growth rates. This calculation requires enormous amounts of computational power and so is left for future research. The fact that adding population growth to the estimating equation does not substantially change the results alleviates this concern somewhat.
} 


$$
\log u_{t+1}(c)-\log u_{t}(c)=\log y_{t+1}(c)-\log y_{t}(c)=\alpha_{1}+\alpha_{2} \log \sum_{S_{c}} L_{c}(s)^{\alpha_{3}}
$$

where

$$
\begin{aligned}
\alpha_{1} & =\frac{1-\gamma_{2}}{\theta} \log \eta+\frac{\gamma_{1}}{\xi} \log \left(\frac{\gamma_{1} / \nu}{\gamma_{1}+\mu \xi}\right) \\
\alpha_{2} & =\frac{\left[1-\gamma_{2}\right]}{\theta} \\
\alpha_{3} & =\frac{\theta \gamma_{1}}{\left[1-\gamma_{2}\right] \xi},
\end{aligned}
$$

and country-level per capita growth is such that, in steady state, $\log y_{t+1}(c)-\log y_{t}(c)=\log y_{t+1}(r)-\log y_{t}(r)$ for all $r \in c{ }^{13}$ The theory therefore predicts that steady-state growth is a function of the following measure of the spatial distribution of population

$$
\sum_{S} L(s)^{\alpha_{3}}
$$

Assuming $\alpha_{2}>0$, then if $0<\alpha_{3}<1$, steady-state growth is maximized when labor is equally spread across space, and if $\alpha_{3}>1$, steady-state growth is maximized when labor is concentrated in one cell. Before estimating 25, we normalize 26 in order to eliminate the effect of the number of cells differing across countries:

$$
\frac{1}{N S} \sum_{S} L(s)^{\alpha_{3}}
$$

where $N S$ is the number of cells in a country. To see what this normalization does, consider two examples. Country A has 4 cells: 2 have population levels $L_{1}$ and 2 have population levels $L_{2}$. Country B is identical to country A, but is quadruple its size: it has 16 cells, of which 8 have population levels $L_{1}$ and 8 have population levels $L_{2}$. The above normalization (27) makes the population distribution measures of countries A and B identical.

To get empirical estimates for $\alpha_{1}, \alpha_{2}$ and $\alpha_{3}$, we use cell population data from G-Econ 4.0 to construct a measure of 27] for four years: 1990, 1995, 2000 and 2005. We focus on countries with at least 20 cells, and for the data on real GDP per capita, we aggregate cell GDP and cell population from the G-Econ data set to compute a measure of real GDP per capita. This gives us 106 countries and 3 time periods.

When estimating (25), we use the between-estimator, i.e., we use the mean of the different variables. We do so because the dependent variable (growth) is rather volatile, whereas the independent variable of interest (the spatial distribution of population) is rather persistent. This suggests that most of the variation should come from differences between countries, rather than from differences within countries. Moreover, 25) is a steady-state relation, so focusing on the average 5-year growth rates seems sensible. Our estimation gives values of $\alpha_{2}=0.00116$ and $\alpha_{3}=2.2$. Using the expressions for $\alpha_{2}$ and $\alpha_{3}$ following (25), this yields $\gamma_{1}=0.319$ and $\gamma_{2}=0.993$, which are the values reported in Table 1 .

If we were to allow for population growth, this would not affect the estimates as long as in the balanced growth path population growth is the same across countries. If, however, population growth rates do differ across countries, equation 25 would become $\log y_{t+1}(c)-\log y_{t}(c)=\alpha_{1}+\alpha_{2} \log \sum_{S_{c}} L_{c}(s)^{\alpha_{3}}+\lambda\left[\log L_{t+1}(c)-\log L_{t}(c)\right]$. Re-

\footnotetext{
${ }^{13}$ Equation 25 is consistent with there being no differences in population growth across countries in a balanced growth path. If we were to allow for such differences, the first part of expression 25 should be written as $\log u_{t+1}(c)-\log u_{t}(c)=\log y_{t+1}(c)-$ $\log y_{t}(c)-\lambda\left[\log L_{t+1}(c)-\log L_{t}(c)\right]$, where $L_{t}(c) \equiv \sum_{S_{c}} L_{t}(s)$. As we will discuss later, this leads to very similar parameter values.
} 
estimating this equation and imposing a value of $\lambda=0.32$, as estimated before, yields very similar results: $\alpha_{2}=$ 0.00103 and $\alpha_{3}=2.6$. This leaves the values of $\gamma_{1}$ and $\gamma_{2}$ virtually unchanged at, respectively, 0.335 and 0.993 .

\subsection{Trade Costs}

We build on Allen and Arkolakis (2014) to calculate trade costs across locations. We discretize the world into $1^{\circ}$ by $1^{\circ}$ grid cells, which means $180 \times 360=64,800$ grid cells in total. A location thus corresponds to a grid cell. To ship a good from location $r$ to $s$, one has to follow a continuous and once-differentiable path $g(r, s)$ over the surface of the Earth that connects the two locations. Passing through a location is costly. We assume that the cost of passing through location $r$ is given (in logs) by

$$
\begin{aligned}
& \log \sigma(r)=\sigma_{\text {rail }} r a i l(r)+\sigma_{n o \_r a i l}[1-\text { rail }(r)]+\sigma_{\text {major_road }} \text { major_road }(r)+\sigma_{\text {other_road }} \text { other_road }(r)+ \\
& \sigma_{n o \_r o a d}[1-\text { major_road }(r)-\text { other_road }(r)]+\sigma_{\text {water }} w a t e r(r)+\sigma_{n o \_w a t e r}[1-\text { water }(r)]
\end{aligned}
$$

where rail $(r)$ equals one if there is a railroad passing through $r$ and zero otherwise, major_road $(r)$ equals one if there is a major road passing through $r$ and zero otherwise, other_road (r) equals one if there is some other road (but no major road) passing through $r$ and zero otherwise, and water $(r)$ equals one if there is a major water route

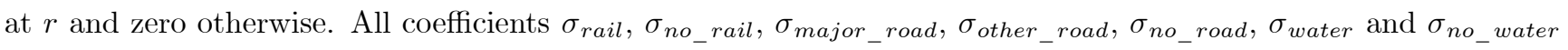
are positive constants, and their values are based on Allen and Arkolakis (2014).

We observe data on water, rail, and road networks at a finer spatial scale than the $1^{\circ}$ by $1^{\circ}$ level. In particular, using data from http://www.naturalearthdata.com/, we can see whether there is a railroad, major road, etc. passing through any cell of size $0.01^{\circ}$ by $0.01^{\circ}$. We aggregate these data up to the $1^{\circ}$ by $1^{\circ}$ grid cell level such that, for instance, rail ( $r$ ) now corresponds to the fraction of smaller cells within cell $r$ that have access to the rail network. We do the same aggregation for the road and water variables ${ }^{14}$

Having $\sigma(r)$, we use the Fast Marching Algorithm to compute the minimum cost between any two cells $r$ and $s:$

$$
\log \varsigma(r, s)=\inf _{g(r, s)} \int_{g(r, s)} \log \sigma(u) d u
$$

where $\int_{g(r, s)} \log \sigma(u) d u$ denotes the line integral of $\log \sigma(\cdot)$ along the path $g(r, s){ }^{15}$

\subsection{Local Amenities and Initial Productivity}

To simulate the model, we also need to know the spatial distribution of $\bar{a}(r) / \bar{u}(c)$ and $\tau_{0}(r)$. We use data at the grid cell level on land $H(r)$ from the National Oceanic and Atmospheric Administration, as well as population $\bar{L}_{0}(r)$ and wages $w_{0}(r)$ as measured by GDP per capita in 2000 (period 0) from G-Econ 4.0, to recover these distributions. Using the equation

$$
w_{0}(r)=\bar{w}\left[\frac{\bar{a}(r)}{\bar{u}(c)}\right]^{-\frac{\theta}{1+2 \theta}} \tau_{0}(r)^{\frac{1}{1+2 \theta}} H(r)^{-\frac{1}{1+2 \theta}} \bar{L}_{0}(r)^{\frac{\alpha-1+\left[\lambda+\frac{\gamma_{1}}{\xi}-[1-\mu]\right] \theta}{1+2 \theta}},
$$

\footnotetext{
${ }^{14}$ Clearly, building roads and rail is endogenous to local development. We abstract from this aspect, but note that major roads and rail lines are in general constructed through geographically convenient locations, a feature of space that is, in fact, exogenous. In any case, the major determinant of the cost of passing through a location is the presence of water.

${ }^{15}$ We apply Gabriel Peyre's Fast Marching Toolbox for Matlab to calculate the minimum costs, taking into account that the Earth is a sphere.
} 
we obtain

$$
\tau_{0}(r)=\bar{w}^{-(1+2 \theta)}\left[\frac{\bar{a}(r)}{\bar{u}(c)}\right]^{\theta} H(r) w_{0}(r)^{1+2 \theta} \bar{L}_{0}(r)^{1-\alpha-\left[\lambda+\frac{\gamma_{1}}{\xi}-[1-\mu]\right] \theta}
$$

for any $r \in S_{c}$. Plugging this into equation (21), we get

$$
w_{0}(r)^{-\theta} \bar{L}_{0}(r)^{\lambda \theta}\left[\frac{\bar{a}(r)}{\bar{u}(c)}\right]^{-\theta}=\left[\bar{u}_{0}^{W}\right]^{-\theta} \kappa_{1} \bar{w}^{-(1+2 \theta)} \sum_{d=1}^{C} \int_{S_{d}} w_{0}(s)^{1+\theta} \bar{L}_{0}(s)^{1-\lambda \theta} H(s) \varsigma(r, s)^{-\theta}\left[\frac{\bar{a}(s)}{\bar{u}(d)}\right]^{\theta} d s .
$$

Given $H(r), \bar{L}_{0}(r)$ and $w_{0}(r)$, and normalizing $\bar{u}_{0}^{W}$ to 1 and $\bar{w}$ to the average wage in the world in 2000, one has to solve equation (29) for $\bar{a}(r) / \bar{u}(c)$. Then one can use equation (28) to obtain $\tau_{0}(r)$. The following lemma says that the values of $\bar{a}(r) / \bar{u}(c)$ and $\tau_{0}(r)$ that satisfy these equations are unique.

Lemma 6 The solution to equations (28) and 29) exists and is unique.

Proof. The existence and uniqueness of a solution to (28) directly follows from the existence and uniqueness of a solution to (29). To prove existence and uniqueness for (29), see again Theorem 2.19 in Zabreyko et al. (1975).

Lemma 6 guarantees the existence and uniqueness of the inversion of the model used to obtain the $\bar{a}(r) / \bar{u}(c)$ and $\tau_{0}(r)$. However, it does not guarantee that we can find a solution using an iterative procedure. At the end of Appendix B we discuss the numerical algorithm we use to find a solution.

Notice that our procedure only identifies $\bar{a}(r) / \bar{u}(c)$, but it cannot be used to tell $\bar{a}(r)$ apart from $\bar{u}(c)$. This is not a problem if mobility restrictions remain unchanged after period 0 . In that case, as can be seen from equation (21), the equilibrium is influenced only by the ratio $\bar{a}(r) / \bar{u}(c)$. However, for counterfactual exercises where we change migration restrictions, the values of $\bar{u}(c)$ change. In that case, we need an estimate of the initial $\bar{u}(c)$. Of course, we also need to have such an estimate if we are interested in the values of local amenities per se. For example, if we were to naively assume that utility was the same everywhere, we would erroneously interpret $\bar{a}(r) / \bar{u}(c)$ to be equal to local amenities. In that case unattractive places in which people are trapped would be mistaken as fantastic places to live. In the next section we explain how we get an estimate of $\bar{u}(c)$ by using data on countries' subjective well-being.

\subsection{Relative Utilities}

We use data on subjective well-being from the Gallup World Poll. Subjective well-being is measured on a Cantril ladder from 0 to 10, where 0 represents the worst possible life and 10 the best possible life the individual can contemplate for himself. This measure is, of course, ordinal, not cardinal. Furthermore, it requires the individual to set her own comparison benchmark when determining what the best possible life, or the worst possible life, might mean. This benchmark might vary across individuals, regions and countries. However, given that Deaton and Stone (2013) and Stevenson and Wolfers (2013) find a relationship between subjective well-being and the log of real income that is similar within the U.S. and across countries, we abstract from these potential differences in

welfare benchmarks across the world. But we still need to transform subjective well-being into a cardinal measure of the level of well-being. In what follows we suggest two ways of doing so.

Recall that in the model the utility of an individual $i$ residing in location $r$ is linear in her real income, namely,

$$
u_{i}(r)=a(r) y_{i}(r)
$$


where real income is $y_{i}(r)=\left[w_{i}(r)+R(r) / \bar{L}(r)\right] / P(r)$. Since we are focusing on a given time period, we have dropped the time subscript in the previous expressions. Then, to make the "ladder" data from the Gallup World Poll comparable to the utility measure in the model, we need to transform subjective well-being into a measure that is linear in income.

\subsubsection{Using existing estimates of the relation between subjective well-being and income}

Deaton and Stone (2013) find that the ladder measure of the subjective well-being of an individual $i$ residing in location $r$ is linearly related to the log of her real income ${ }^{16}$ In particular, they estimate a relation

$$
\tilde{u}_{i}(r)=\rho \ln y_{i}(r)+v(r)+\varepsilon_{i}
$$

where the tilde refers to subjective well-being, as measured by the Cantril ladder, $v(r)$ is a location fixed effect, and $\varepsilon_{i}$ is a random variable with mean zero. Whereas the ladder measure is linear in the log of real income, our utility measure in 30 is linear in the level of real income. To make 31 consistent with our model, take logs of [30, multiply by $\rho$, and allow for an idiosyncratic component to log utility. This yields

$$
\rho \ln u_{i}(r)=\rho \ln y_{i}(r)+\rho \ln a(r)+\varepsilon_{i} .
$$

Equations (32) and (31) imply the following relation between utility as defined in our model, $u_{i}(r)$, and utility as defined by subjective well-being, $\tilde{u}_{i}(r)$,

$$
u_{i}(r)=e^{\psi \tilde{u}_{i}(r)}
$$

where $\psi=1 / \rho$. Given the structure of our model, one potential issue with estimating (31) if we only were to use cross-country or cross-regional data is endogeneity: a location with a higher utility attracts more people and therefore affects the amenity levels through $a(r)=\bar{a}(r) \bar{L}(r)^{-\lambda}$. However, if 31] is estimated for a given time period using the cross-sectional variation and including locational fixed effects, this is less of a concern. Using person-level data, Deaton and Stone (2013) estimate $\rho$ to be around 0.55, which implies a value of $\psi$ of 1.8.

\subsubsection{Using data on actual and desired migration}

Another way of obtaining an estimate of $\psi$ is by using information on actual and desired migration. We proceed in two steps. First, we derive an expression that relates the utility difference between two locations in our model to desired migration and the barriers to migration. Second, using equation (33), we use data on subjective well-being and on desired migration from the Gallup World Poll, to estimate $\psi$.

Step 1: Relate utility differences to desired migration.

We start by relating utility differences between two locations and desired migration. As is standard in the migration literature (Docquier et al., 2012; Behrens et al., 2013), we slightly adjust our model to allow for idiosyncratic taste differences across individuals and locations. Without such taste differences, when individuals in location $r$ are asked whether they want to migrate to location $s$, either all of them or none of them should respond in the affirmative. Since survey data do not exhibit such extreme patterns, allowing for idiosyncratic taste differences is reasonable.

\footnotetext{
${ }^{16}$ See also Kahneman and Deaton (2010).
} 
We can then define the utility of an individual $i$ born in location $r$ who moves to location $s$ as

$$
u_{m}^{i}(r, s)=u(s)-m(r, s)+\varepsilon^{i}(s)
$$

where $u(s)$ is the utility of residing in location $s$, common across all individuals; $m(r, s)$ represent the barriers to migrating from $r$ to $s$, with $m(r, s)=0$ if $r$ and $s$ are in the same country; and $\varepsilon^{i}(s)$ represents idiosyncratic taste differences, distributed i.i.d. across individuals and locations according to an extreme value distribution with mean zero and variance $\pi^{2} \Omega^{2} / 6$, with a higher value of $\Omega$ indicating greater taste heterogeneity ${ }^{17}$ The probability that an individual born in $r$ prefers location $s$ over all other locations can then be written as

$$
\operatorname{Pr}\left(u_{m}(r, s)=\max _{k} u_{m}(r, k)\right)=\frac{\exp ([u(s)-m(r, s)] / \Omega)}{\sum_{k} \exp ([u(k)-m(r, k)] / \Omega)} .
$$

In equilibrium, this corresponds to the share of population born in location $r$ that lives in location $s$ :

$$
\frac{L(r, s)}{L(r)}=\frac{\exp ([u(s)-m(r, s)] / \Omega)}{\sum_{k} \exp ([u(k)-m(r, k)] / \Omega)} .
$$

By analogy, we can derive $L(r, r) / L(r)$ :

$$
\frac{L(r, r)}{L(r)}=\frac{\exp (u(r) / \Omega)}{\sum_{k} \exp ([u(k)-m(r, k)] / \Omega)} .
$$

Taking logs and dividing expressions $(36)$ and 37 yields

$$
\ln \frac{L(r, s)}{L(r, r)}=\frac{u(s)-u(r)-m(r, s)}{\Omega} .
$$

Now suppose we eliminate legal migration barriers. Of course, some migration barriers would still persist. Denote those remaining migration barriers by $m^{0}(r, s)$. To simplify, assume that $m^{0}(r, s)=m^{0}{ }^{18}$ In that case, the above expression becomes

$$
\ln \frac{L^{0}(r, s)}{L^{0}(r, r)}=\frac{u(s)-u(r)-m^{0}}{\Omega},
$$

where the 0 superscript refers to variables in the absence of migration barriers. Rewriting (39), we can relate utility differences to desired migration and the migration barriers using

$$
u(s)-u(r)=\Omega\left[\ln L^{0}(r, s)-\ln L^{0}(r, r)\right]+m^{0} .
$$

\section{Step 2: Estimation}

According to our model, utility in 40 depends linearly on the level of income 19 whereas the subjective utility measure from the Gallup World Poll depends linearly on the log of income, as shown by Deaton and Stone (2013).

\footnotetext{
${ }^{17}$ We interpret this barrier as a quota restriction that selects the individuals who are more willing to migrate. We model it here as a cost faced by the marginal migrant that makes him indifferent to migrating. This cost $m(r, s)$ is selected by the target country to keep relative utility levels constant and is rebated to the number of migrants in the quota.

${ }^{18}$ Alternatively assuming that $m^{0}(r, s)$ is a linear function of $m(r, s)$ yields similar parameter estimates.

${ }^{19}$ Interpreting the utility in equation 40 as depending linearly on income is not only consistent with our model, it is also in line with work by Grogger and Hanson (2011), who estimate a linear utility equation similar to 40 to study the relation between income and migration.
} 
This implies that $u(r)=e^{\psi \tilde{u}(s)}$, so that we can rewrite 40 as

$$
e^{\psi \tilde{u}(s)}-e^{\psi \tilde{u}(r)}=\Omega\left[\ln L^{0}(r, s)-\ln L^{0}(r, r)\right]+m^{0} .
$$

The three parameters $\psi, \Omega$ and $m^{0}$ in $(41)$ can then be estimated by minimizing

$$
\min _{\psi, \Omega, m^{0}} \sum_{r}\left[e^{\psi \tilde{u}(s)}-e^{\psi \tilde{u}(r)}-\Omega\left[\ln L^{0}(r, s)-\ln L^{0}(r, r)\right]-m^{0}\right]^{2} .
$$

We estimate (42) using aggregate data at the country level. To be more precise, for the 150 countries in our data set $\tilde{u}$ refers to subjective well-being on the Cantril ladder ${ }^{20} L^{0}(r, s)$ is the number of people from location $r$ who either reside in location $s$ or desire to reside in location $s$; and $L^{0}(r, r)$ is the number of people from location $r$ who want to stay in location $r$. Since migration costs are assumed to be zero within countries, which implies that utility equalizes across locations within a country, we estimate equation 42 with locations aggregated by country. The country-level data on subjective well-being and desired migration come from the Gallup World Poll, whereas the data on actual migration come from Artuc et al. (2012). Note that, for each of the countries, we only know how many people want to migrate to the most popular destination country. Hence, for each origin country we only have information on one destination country ${ }^{21}$

One issue with the data is that, in some cases, taste differences common to all individuals of a certain country may render the difference in average utility a poor measure of the desire to migrate. For example, the favorite destination of Afghanistan is Iran (religious links); the favorite destination of Suriname is the Netherlands (colonial links); and the favorite destination of Luxembourg is Portugal (migration links going back to the mid-1960s). To minimize this issue, we exclusively focus on destination countries that are the favorite of a minimum number of countries. When restricting our focus to countries that are favorite destinations shared by at least 7 countries, we are left with 122 observations and estimate a value of $\psi=2.8$. A more stringent restriction of the favorite destination being shared by at least 10 countries limits the number of observations to 97 and yields an estimate of $\psi=1.2{ }^{22}$ The average of these two estimates is very similar to the 1.8 figure found by Deaton and Stone (2013), so we take that value for $\psi$.

\subsection{Computing Actual Local Amenities and Counterfactual Migration Scenarios}

Local amenities. The inversion of the model described in Section 4.4 uses wage and population data to identify $\bar{a}(r) / \bar{u}(c)$, whereas the data on subjective well-being give us an estimate of utility, $\bar{u}(c)=e^{1.8 \tilde{u}(c)}$. Together, this allows us to identify the amenity level of a location $r$,

$$
\bar{a}(r)=e^{1.8 \tilde{u}(c)} \frac{\bar{a}(r)}{\bar{u}(c)} .
$$

\footnotetext{
${ }^{20}$ We include a world map with the Cantril ladder data in Appendix A.3.

${ }^{21}$ Though the data are at the country-level, it is important to point out that the information on how many people want to migrate is based on individual-level survey data. It is therefore reasonable to assume that people do not take into account general equilibrium effects on utility through, for example, a change in amenities, when answering the question on whether they want to migrate. If not, our estimation would be biased.

${ }^{22}$ The favorite destinations shared by at least 7 countries are Australia, France, Germany, Russia, South Africa, Spain, the U.K. and the U.S. When restricting the list to include only destinations shared by at least 10 countries, Australia and Russia drop out.
} 
Counterfactual migration scenarios. We now describe how to simulate the model under counterfactual migration restrictions.

\section{Keeping mobility restrictions unchanged}

Suppose we want to simulate the model over many periods, keeping current mobility restrictions unchanged. This amounts to keeping relative utility differences across countries constant. In that case, we use the values $\bar{a}(r) / \bar{u}(c)$ from the original inversion and keep them constant over time. Importantly, since the entire simulation is based on the original values $\bar{a}(r) / \bar{u}(c)$, this exercise makes no use of the data on subjective well-being and is thus independent of the particular way in which we map subjective well-being in the data to utility in the model.

Free mobility

Now suppose we want to see what happens under free mobility. This requires reshuffling the population such that utility equalizes across all locations. In that case, rather than simulating the model forward using the values $\bar{a}(r) / \bar{u}(c)$ from the original inversion, we simulate the model using the values $e^{1.8 \tilde{u}(c)} \bar{a}(r) / \bar{u}(c)$, where $e^{1.8 \tilde{u}(c)} \bar{a}(r) / \bar{u}(c)$ is nothing else than $\bar{a}(r)$. Hence, this amounts to substituting $\bar{a}(r) / \bar{u}(c)$ by $\bar{a}(r)$, which is the same as imposing utility equalization across space.

\section{Partial mobility}

Of course, we might also be interested in intermediate cases, where mobility restrictions are relaxed but not completely eliminated. More generally, and by analogy with the previous argument, we can compute an equilibrium using $e^{\psi \tilde{u}(c)} \bar{a}(r) / \bar{u}(c)$, where $\psi \in[0,1.8]$. The two cases above correspond to the two extremes. If $\psi=0$, then $e^{0 \tilde{u}(c)} \bar{a}(r) / \bar{u}(c)$ is equal to the values $\bar{a}(r) / \bar{u}(c)$ of the original inversion, so we are in the case of keeping migration restrictions unchanged. If $\psi=1.8$, we are in the case of free mobility. If $\psi$ is strictly in between 0 and 1.8 , we have partial liberalization of mobility restrictions. The closer $\psi$ is to 1.8 , the greater is the liberalization of migration restrictions. Note that all these exercises keep the ranking of migration restrictions (as reflected by the relative utility levels in the observed subjective well-being data) unchanged. In principle, we could also consider negative values of $\psi$, implying a strengthening of migration restrictions around the world, or values of $\psi>1.8$, which would amount to migration incentives rather than restrictions.

\subsection{Description of Computational Algorithm}

With the values of the structural parameters, trade costs, land, amenities and initial productivity in hand, we can simulate the model from period 1 onward. The following algorithm is used to calculate the equilibrium population distribution in period $t$. Guess some initial population distribution $\bar{L}_{t}^{0}(\cdot)$ and a world utility level $\bar{u}_{t}^{W}$. Plug $\bar{L}_{t}^{0}(\cdot)$ into the right-hand side of equation (21), calculate the left-hand side, and solve for the distribution of population; call this $\bar{L}_{t}^{1}(\cdot)$. Then compute the distance between $\bar{L}_{t}^{1}(\cdot)$ and $\bar{L}_{t}^{0}(\cdot)$, defined as

$$
d i s t_{t}^{1}=\int_{S}\left[\bar{L}_{t}^{1}(r)-\bar{L}_{t}^{0}(r)\right]^{2} d r
$$

If $d i s t_{t}^{1}<\varepsilon$ where $\varepsilon$ is an exogenously given tolerance level, stop. Otherwise, plug $\bar{L}_{t}^{1}(\cdot)$ into the right-hand side of 21 , obtain the updated population distribution $\bar{L}_{t}^{2}(r)$, and compute $d i s t_{t}^{2}$, defined analogously to dist $t_{t}^{1}$. Continue the procedure until $d i s t_{t}^{i}<\varepsilon$ for some $i$. 
The following lemma shows that this algorithm converges to the equilibrium population distribution whenever the equilibrium exists, and is unique.

Lemma 7 If $\alpha / \theta+\gamma_{1} / \xi \leq \lambda+1-\mu$ (the sufficient condition for the existence and uniqueness of the equilibrium in Lemma 3), the algorithm described above converges to the equilibrium distribution of population. That is,

$$
\bar{L}_{t}(r)=\lim _{i \rightarrow \infty} \bar{L}_{t}^{i}(r)
$$

Proof. See again Theorem 2.19 in Zabreyko et al. (1975).

In an outer loop we then find the world utility level $\bar{u}_{t}^{W}$ that solves the world labor market clearing

$$
\int_{S} H(r) \bar{L}_{t}(r) d r=\bar{L}
$$

Finally, technology can be updated using (19). Hence, the population distribution can be calculated for an arbitrary number of successive periods $t=1,2, \ldots, T$.

\section{The Geographic Evolution of the World Economy}

The aim of this section is three-fold. First, we want to enhance our understanding of the relation between space and growth. Our model has predictions for the future evolution of the spatial distribution of population and productivity, as well as for the economy's aggregate growth rate. As we will show, these predictions will depend crucially on how easily people can move across countries. Second, we want to understand the welfare impact of relaxing migration restrictions and how doing so changes the distribution of economic activity in the balanced growth path. Finally, we want to analyze the impact of location-specific shocks, with a particular focus on the rise in sea levels due to global warming.

\subsection{Benchmark Calibration}

As explained in Section 4.4, we use cell-level data on land, population, wages and trade costs to recover amenities and productivity. Whenever time-variant, the data are mostly for 2000 (see Appendix C for more details). All outcomes are plotted in natural logarithms. Figure 1 presents the results from the inversion exercise using actual migration frictions to calculate the fundamental productivities and amenities. By 'fundamental' we refer to the part of productivity and amenities that does not depend on population density. That is, it does not take into account the positive agglomeration economies that benefit productivity and the negative congestion effects that hurt amenities.

Fundamental productivity exhibits the expected patterns (see Figure 1 1 ). Productivity is generally high in North America, Europe and Japan. When we look within countries, the main cities tend to display particularly high levels of productivity. Mexico, for example, exhibits relatively low productivities, except for Mexico City and Monterrey. In China, Beijing and Shanghai have clearly higher fundamental productivities than the rest of the country. This may reflect rules and regulations, as well as local educational institutions that lead to higher skilled populations. It is also well known that cities often tend to attract more productive workers. In that sense it should not come as a surprise that urban areas stand out as places with high fundamental productivities. Clearly, since 
we are abstracting from local capital investments, fundamental productivities also reflect the local stock of capital, which tends to be larger in cities and, more broadly, in developed economies.

As for fundamental amenities (see Figure 1 $\mathrm{b}$ ), the highest values can be found in South America, and in particular Brazil. North America also enjoys high fundamental amenities, particularly in urban areas. In Europe, Scandinavia and parts of France exhibit high amenities, while Portugal and Southern Europe fare worse. Thailand and Southeastern Australia are also desirable places to live, while some of the lowest amenities are found in Africa and Siberia. To validate our identification strategy for amenities, it is worthwhile to correlate our estimates of amenities with commonly used exogenous measures of quality of life. We therefore collect data on different measures of geography (distance to oceans, distance to water, elevation and vegetation) and climate (precipitation and temperature). The correlations between our estimates of amenities and these measures are consistent with the literature. For example, we find that people like living close to water, prefer higher average temperatures, and precipitation (Morris and Ortalo-Magné, 2007; Albouy et al., 2014). Qualitatively the results do not depend on whether we look at all cells of the world, at only cells in the U.S., or at randomly drawn cells across countries. This suggests that our methodology of using data on subjective well-being to identify amenities performs well. To reinforce this point further, placebo correlations based on alternative identification strategies of amenities yield correlations that are no longer the same across countries and within countries. Appendix A.2 presents these correlations and provides further details.

Figure 1: Benchmark Calibration: Results from the Inversion

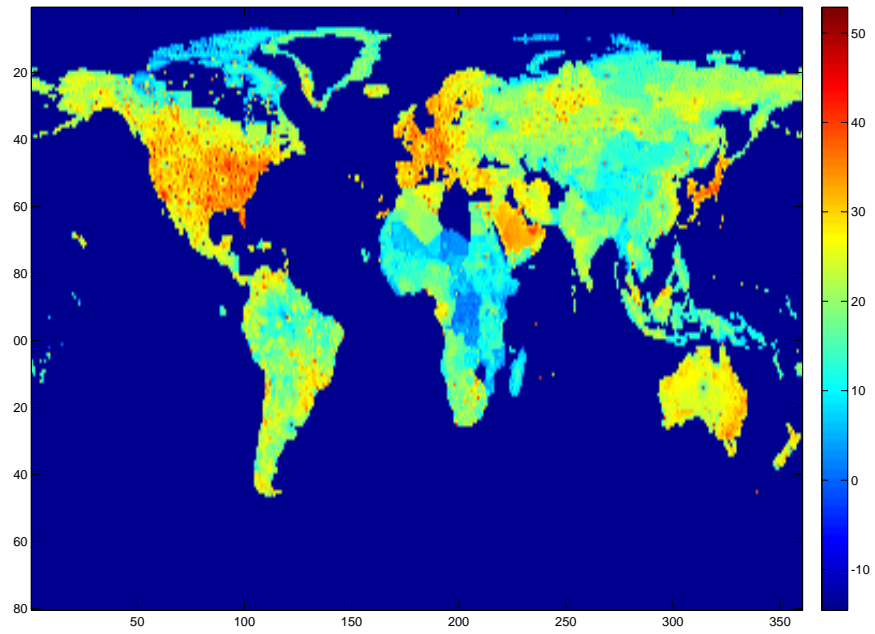

a. Fundamental Productivities: $\tau_{0}(r)$

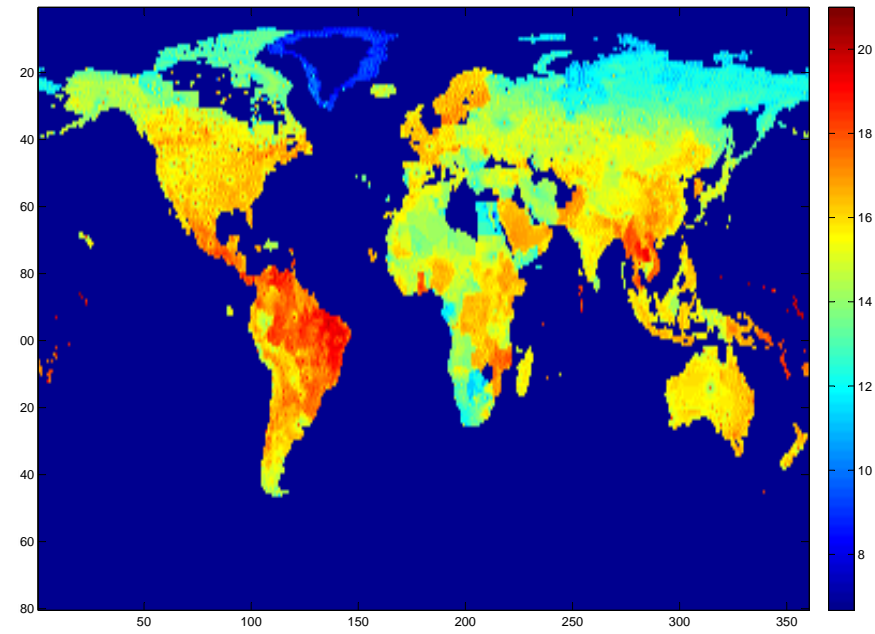

b. Fundamental Amenities: $\bar{a}(r)$

Figure 2 (different panels) maps population, productivity, amenities, and real income per capita. In the case of amenities, the map now presents the overall level of amenities, $\bar{a}(r) \bar{L}_{t}(r)^{-\lambda}$, which depends on population and so takes into account congestion. That is, it takes into account the negative effect that high density imposes on the amenities experienced by residents. We also present average productivity in each cell, which, following the theory, is defined as $\left[\tau_{t}(r) \bar{L}_{t}(r)^{\alpha}\right]^{\frac{1}{\theta}}$. This productivity includes the positive local agglomeration effect with elasticity $\alpha$, and takes into account that each location draws productivities from a Fréchet distribution. In contrast to Figure 1. these are equilibrium outcomes in the first period of the model.

A first observation is that the correlation between population density and productivity across countries is 
Figure 2: Equilibrium in Benchmark Calibration (Period 1)

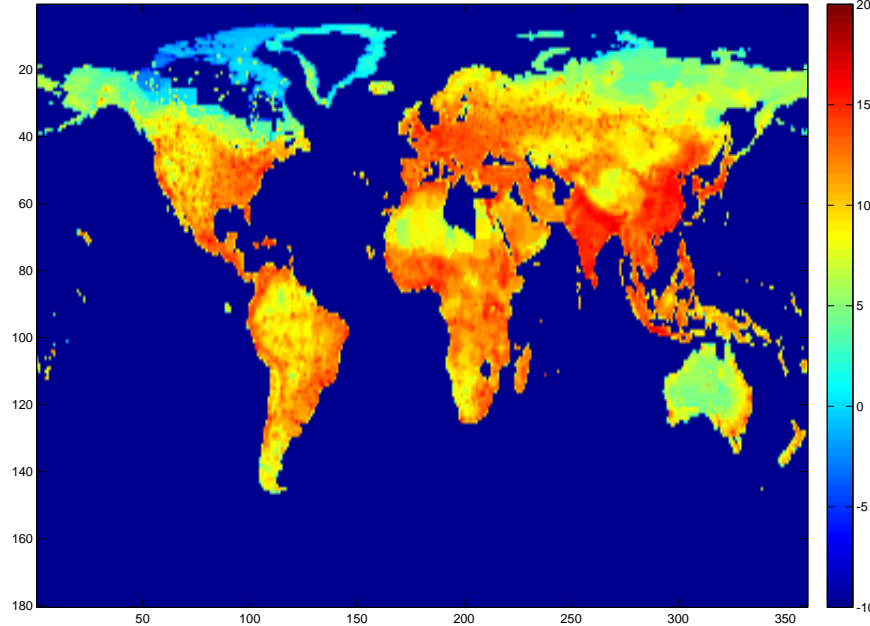

a. Population Density

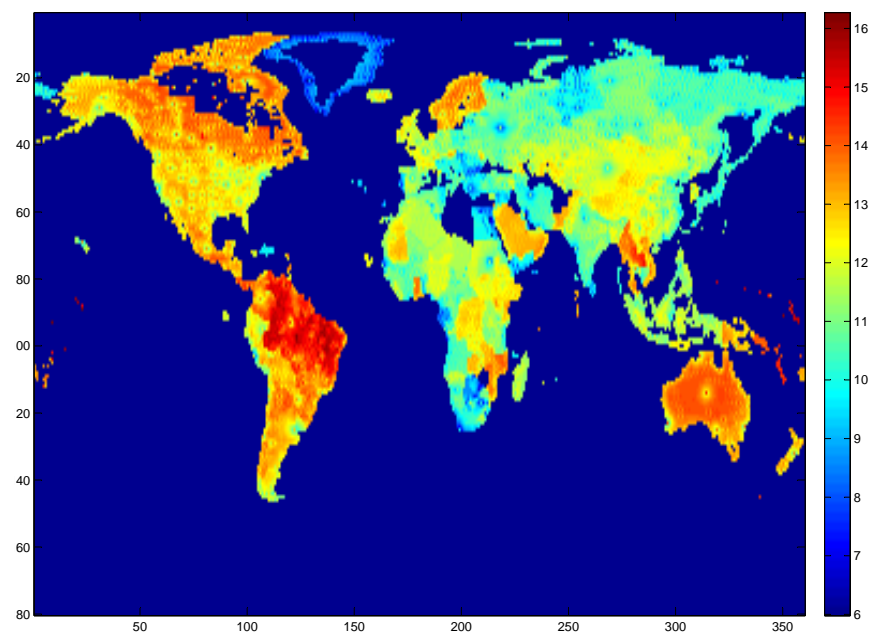

c. Amenities: $\bar{a}(r) \bar{L}_{t}(r)^{-\lambda}$

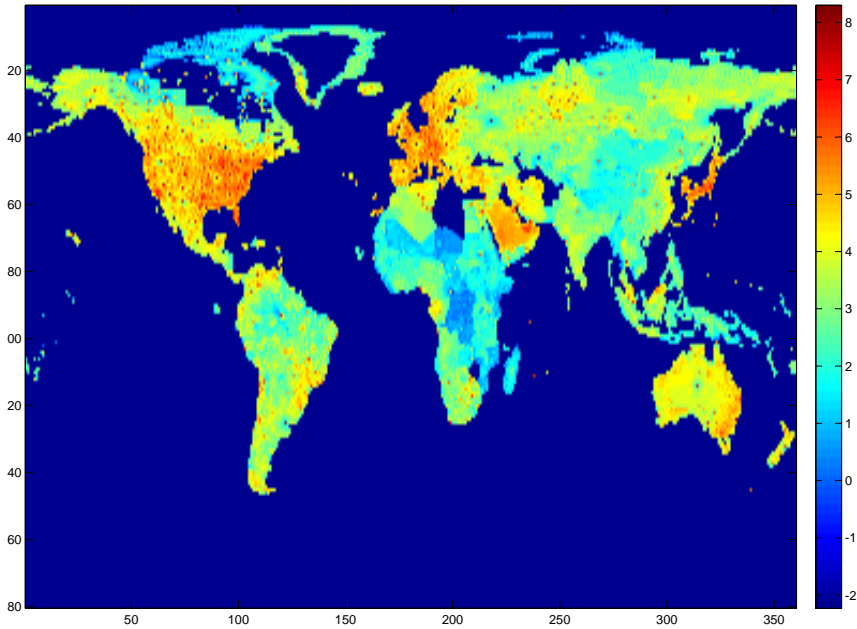

b. Productivity: $\left[\tau_{t}(r) \bar{L}_{t}(r)^{\alpha}\right]^{\frac{1}{\theta}}$

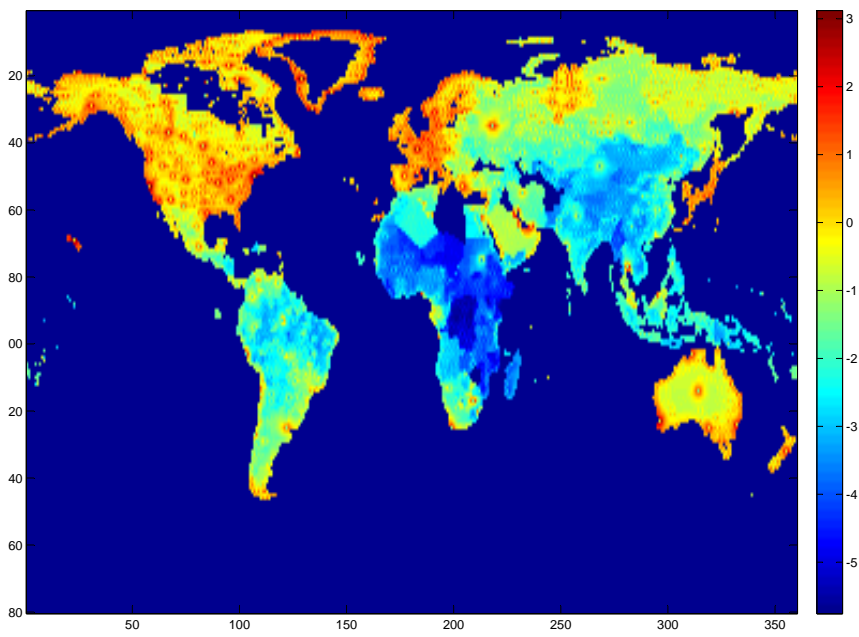

d. Real Income per Capita

not that strong: there are some densely populated countries with high productivity levels, such as many of the European countries, but there are also some densely populated countries with low productivity levels, such as some of those located in sub-Saharan Africa. Countries that are densely populated in spite of having low productivity must either have low levels of utility (e.g., sub-Saharan Africa) or high levels of amenities (e.g., Latin America) ${ }^{23}$ A second observation is that the same relation between population density and productivity is much stronger across locations within countries, where the high-productivity areas typically tend to be the metropolitan areas. This is not surprising: since there are no utility differences across locations within countries, the negative congestion effects from living in densely populated areas have to be compensated by either higher productivity or better amenities. A third observation suggests that both reasons play a role. Metropolitan areas have higher productivity but lower real income per capita, compared to less dense neighboring locations. The lower real income of metropolitan

\footnotetext{
${ }^{23}$ See also the map of the subjective welfare measures in Appendix A.3.
} 
areas suggests that they must enjoy higher amenity levels (in spite of the negative effect of density on amenities) as we highlighted above. A fourth observation is that locations close to metropolitan areas tend to have lower productivity relative not only to metropolitan areas but also to the rest of the country. Since those locations have cheaper access to goods but are often not more densely populated compared to the rest of the country, they must either have lower productivity or worse amenities.

Now consider the evolution of this economy over time, when we keep utility differences across countries unchanged. We interpret this exercise as being equivalent to keeping migratory policy across countries unchanged. As we explained before, for this exercise we do not use the data on subjective well-being ${ }^{24}$ Figure 3 (different panels) maps the predicted distributions of population, productivity, amenities and real income per capita in period 600, at which point the economy has converged to its balanced growth path. Videos that show the evolution of these variables over time and over space are available in an Online Appendix ${ }^{25}$ To visualize the changes over time, we should compare Figure 3, which represents the year 2600, with Figure 2, which represents the year 2000.

Clearly, over time the correlation between population and productivity across countries becomes much stronger. As predicted by the theory, in the long run, high-density locations correspond to high-productivity locations, while in today's world this relation is still largely absent. This can easily be seen when comparing the maps for period 1 and period 600. While in period 1 the population and the productivity maps look quite different, by period 600 they look very similar. As shown by the dashed-blue curve in the middle panel of Figure 4 which presents the correlation of population density and productivity over time, the correlation increases from around 0.3 in period 0 to 1.0 by period 600 . Note, however, that the correlation between population density and real GDP, which is driven not only by productivity but also by local prices, and therefore transport costs and geography, also grows but is never higher than 0.8. The dashed-blue curve in the left panel of Figure 4 shows this. Another remark is that the high-productivity, high-density locations 600 years from now correspond to today's low-productivity, high-density locations, mostly countries located in sub-Saharan Africa, South Asia and East Asia. In comparison, most of today's high-productivity, high-density locations in North America, Europe, Japan and Australia fall behind in terms of both productivity and population.

This productivity reversal can be understood in the following way. The high population density in some of today's poor countries implies high future rates of innovation in those countries. To keep their relative utility levels from increasing, population in those countries must increase, leading to greater congestion costs and worse amenities. As a result, today's high-density, low-productivity countries end up becoming high-density, highproductivity, high-congestion and low-amenity countries, whereas today's high-density, high-productivity countries end up becoming low-density, low-productivity, low-congestion and high-amenity countries. As can be seen in Figure 4 given that the correlation between population density, productivity and real income per capita becomes very high, it must be that low-density, high-utility countries have high amenities.

These dynamics imply a reallocation of population from high-utility to low-utility countries. In principle this could be driven by decreased migration from low- to high-utility countries or by increased migration from highto low-utility countries. It is easier to think about the former if we want to interpret this exercise as one where

\footnotetext{
${ }^{24}$ As explained briefly in the theory section, the logic behind this argument is that the migration quota determines the number of migrants who are allowed to cross the border. Suppose the utility in the receiving country goes up, then the quota is relaxed, so that more people will enter, thereby lowering utility in the host country until the relative utility is unchanged. Now suppose the utility in the sending country goes up, then the host country can make the quota more stringent, so that fewer people will enter, once again leaving the relative utility unchanged. That is, we assume, admittedly without modeling the corresponding political economy process, that countries impose migration quotas according to relative welfare levels.

${ }^{25}$ All videos can be viewed at https://www.princeton.edu/ ${ }^{\sim}$ erossi/videos.html
} 
Figure 3: Equilibrium Keeping Migratory Restrictions Unchanged (Period 600)

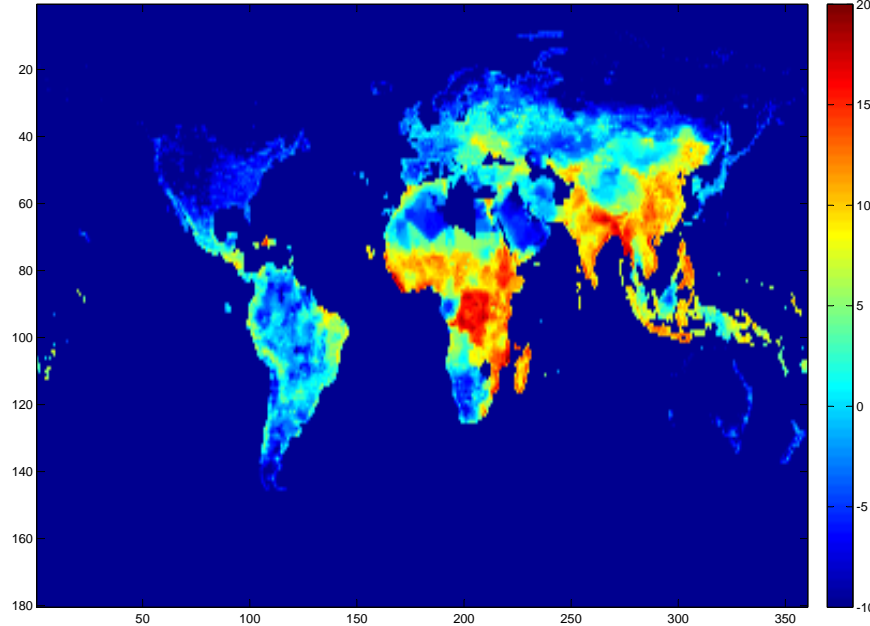

a. Population Density

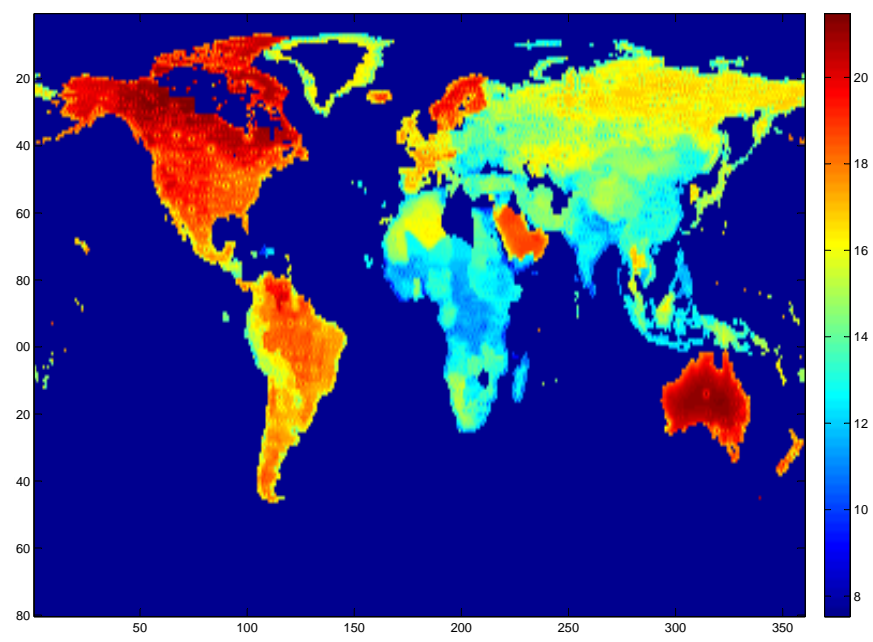

c. Amenities: $\bar{a}(r) \bar{L}_{t}(r)^{-\lambda}$

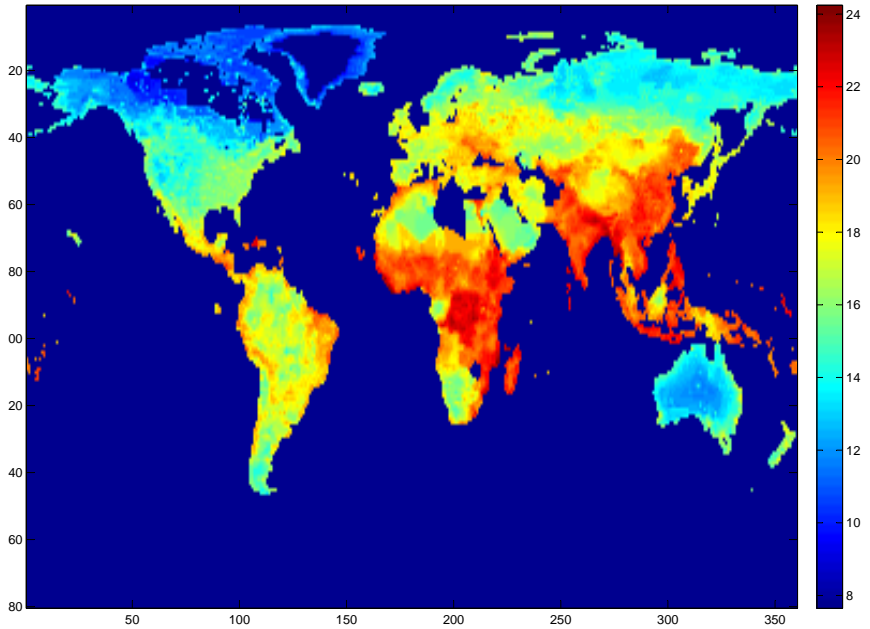

b. Productivity: $\left[\tau_{t}(r) \bar{L}_{t}(r)^{\alpha}\right]^{\frac{1}{\theta}}$

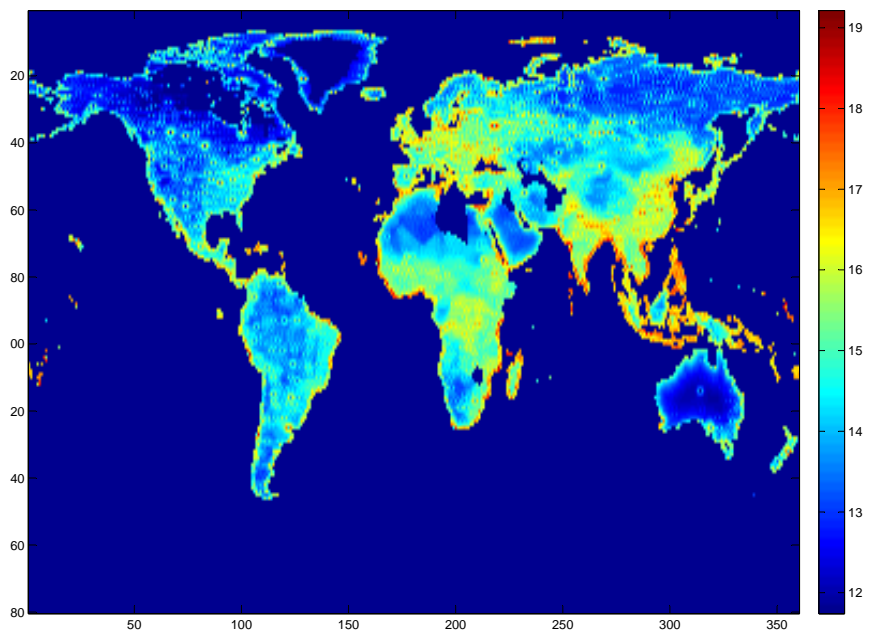

d. Real Income per Capita

we keep existing migratory policy unchanged. As increased innovation pushes up relative utility in low-utility countries, fewer people want to migrate, but a greater share of those who want to migrate are kept out, so that utility differences do not go down. Over time, this reallocation of population peters out. As predicted by Lemma 4. decreasing returns to innovation, together with congestion costs, imply that, in the long run, the distribution of population reaches a steady state in which all locations innovate at the same constant rate.

Most of our discussion so far has focused on the changing differences across countries, but there are also interesting differences within countries. When focusing on the population distribution within countries, we observe that as the population share of North America and Europe declines, the locations that better withstand this decline are the coastal areas, which benefit from lower transport costs and thus higher real income. In the countries whose population shares are increasing, such as China, India and parts of sub-Saharan Africa, there is less evidence of coastal areas gaining. 
Figure 4: Correlations with Different Migration Restrictions and Coastal Flooding Scenarios
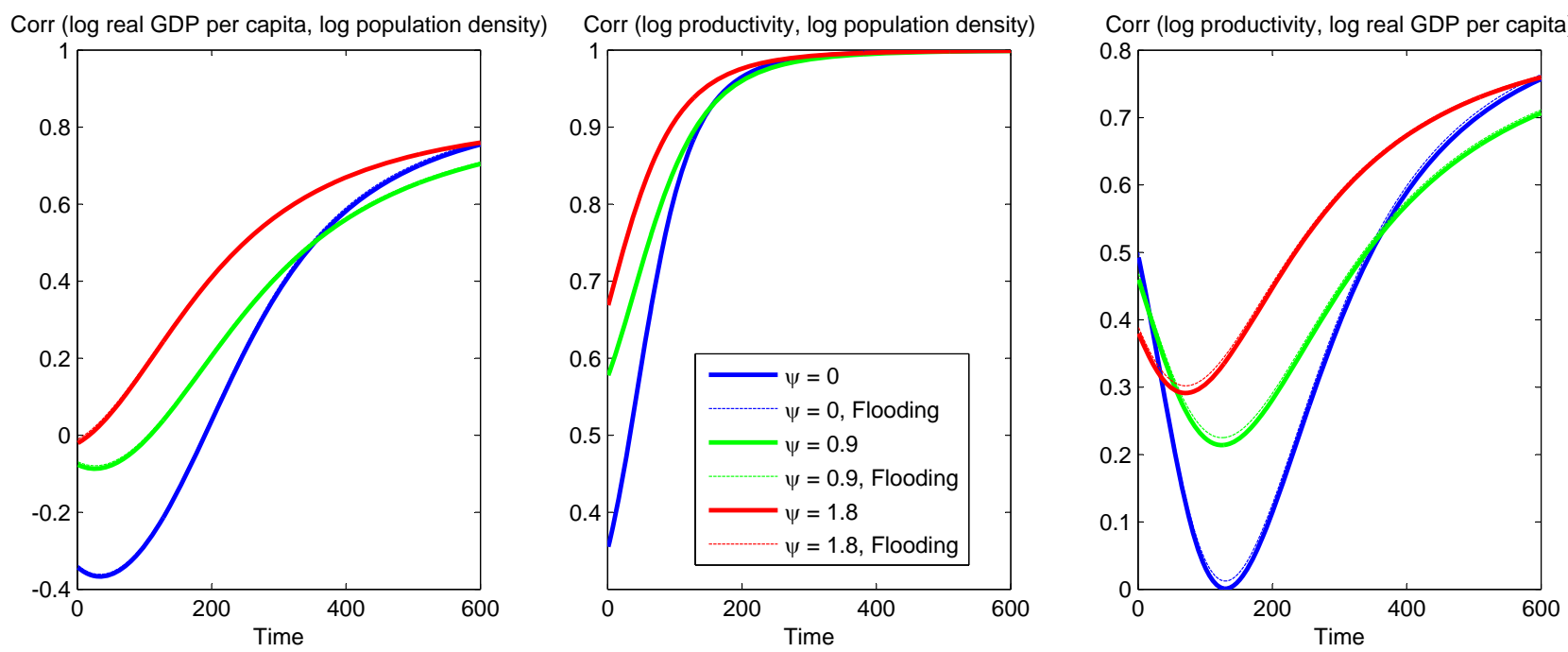

Figure 5 presents the average growth rates and levels of productivity, real output and welfare for different scenarios ${ }^{26}$ The benchmark calibration, which leaves mobility restrictions unchanged, corresponds to $\psi=0$. Consistent with the argument above, Figure 5 shows that the average growth rates of productivity, real GDP per capita and utility converge to constants. Note that the long-run growth rate in real GDP per capita is higher than the initial growth rate in real GDP per capita, whereas the opposite is true for the growth rate in utility. Recall that the growth rate in utility is equal to the growth rate in real GDP per capita minus $\lambda$ times the growth rate of population. What happens is that initially the correlation between the growth in real GDP per capita and the growth in population is negative - many of the high-growth places in richer countries are losing population so that the growth rate in utility is greater than the growth rate in GDP per capita. In the long run, when the steady state is reached, there is no more reallocation of population across space, so that real GDP per capita and utility grow at the same rates.

\subsection{Evaluating Mobility Restrictions}

We now analyze the effect of completely or partially relaxing existing migration restrictions. We start with the free mobility scenario and then present some calculations for scenarios with partial mobility where $\psi$ is in between 0 (current restrictions) and 1.8 (free mobility).

\subsubsection{Free migration}

In this exercise we start off with the benchmark calibration in period 0 , and then reallocate population such that utility equalizes across countries from period 1 onward. With free migration, people move from low-utility countries to high-utility countries. This reduces utility differences across countries because of greater land congestion and lower amenities in high-utility countries (and the opposite in low-utility countries). With enough migration, utility

\footnotetext{
${ }^{26}$ World average productivity at time $t$ is defined as $\int_{S}\left[H(r) \bar{L}_{t}(r) / \bar{L}\right]\left[\tau_{t}(r) \bar{L}_{t}(r)^{\alpha}\right]^{\frac{1}{\theta}} d r$, i.e., the population-weighted average of locations' mean productivity levels. World average real GDP at $t$ is defined analogously as $\int_{S}\left[H(r) \bar{L}_{t}(r) / \bar{L}\right] y_{t}(r) d r$. The growth of utility is measured as the growth in $\bar{u}_{t}^{W}$.
} 
Figure 5: Growth Rates and Levels with Different Migration Restrictions and Coastal Flooding Scenarios
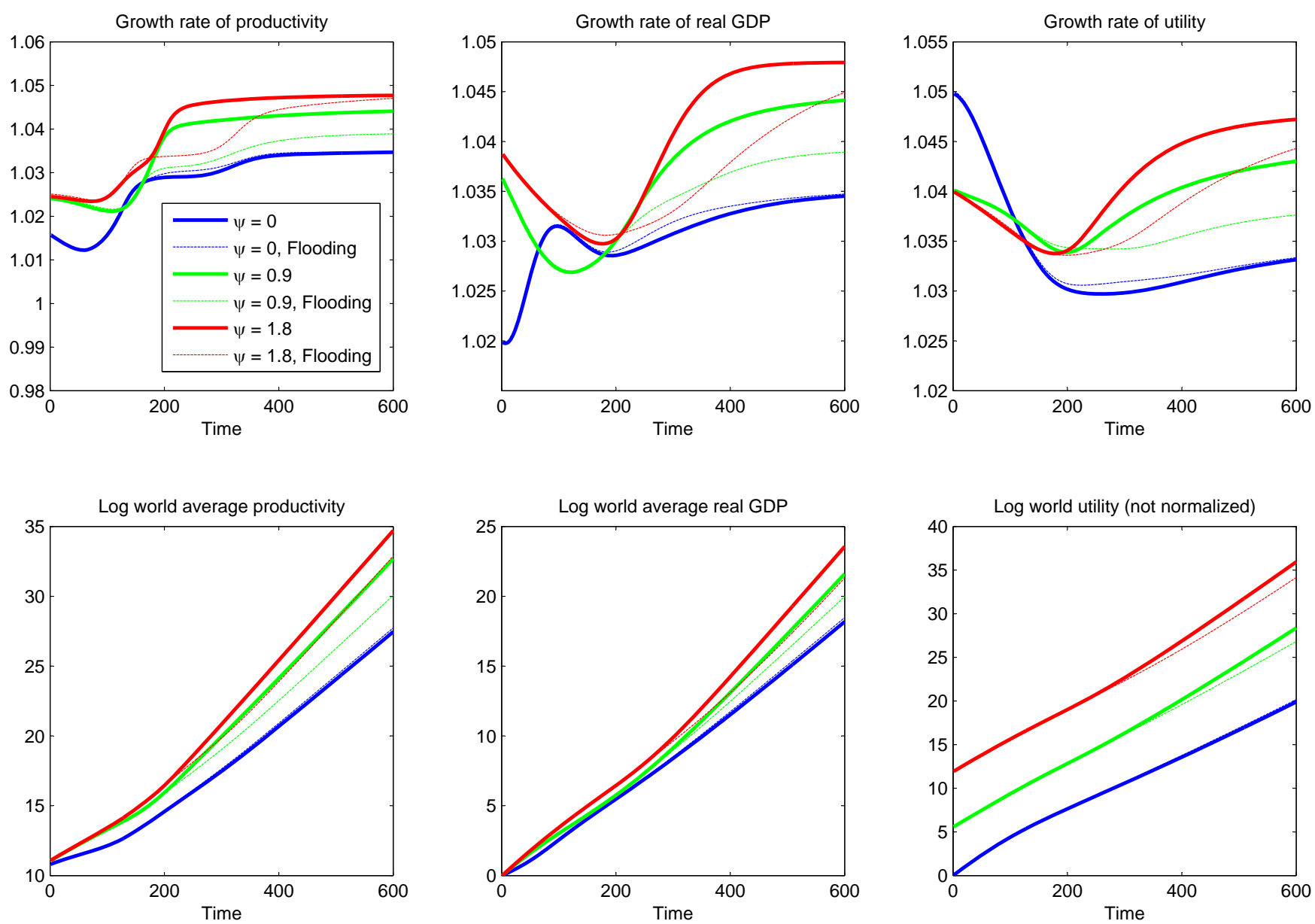

equalizes across all countries. This reallocation of population across countries does not only have a static effect; it also has a dynamic effect by putting the economy on a different dynamic path.

Figure 6 and Figure 7 map the distributions of population, productivity, amenities and real income per capita for period 1 and period 600, under the assumption that people are freely mobile across countries ${ }^{27}$ Compared to the exercise where we kept migratory restrictions unchanged, several observations stand out. First, migration increases the initial correlation between population density and productivity across countries, so that we see fewer countries where high density and low productivity coexist. In the middle panel of Figure 4 we see that in the case of complete liberalization $(\psi=1.8)$ the initial correlation between density and productivity increases from around 0.3 to nearly 0.7 . Second, this initial effect has important dynamic consequences. Because today's poor countries lose population through migration, they innovate less. As a result, and in contrast to the previous exercise, no productivity reversal occurs between the U.S., India, China and sub-Saharan Africa. Third, this absence of a large-scale productivity reversal does not mean that relative productivities across countries remain unchanged. Some countries, such as Venezuela, Brazil and Mexico, start off with relatively high utility levels but relatively low productivity levels. This means they must have high amenities. Because of migration, they end up becoming the

${ }^{27}$ For videos that show the gradual evolution over time and over space, see the Online Appendix 
world's densest and most productive countries. Fourth, migration changes the determination of the development path of the world and thereby increases the growth rate in the balanced growth path, as is clear from Figure 5. Fifth, within countries, there is stronger evidence of an increasing concentration of the population in coastal areas, compared to the benchmark case. The greater concentration of population within countries, which was already apparent in the benchmark case, is now reinforced by greater migration across countries. Sixth, the importance of coastal areas becomes even more apparent when we look at real income per capita. The fact that locations close to coastal areas have lower real income per capita but high population suggest that those locations have higher amenities than the coastal areas themselves.

Figure 6: Equilibrium with Free Migration (Period 1)

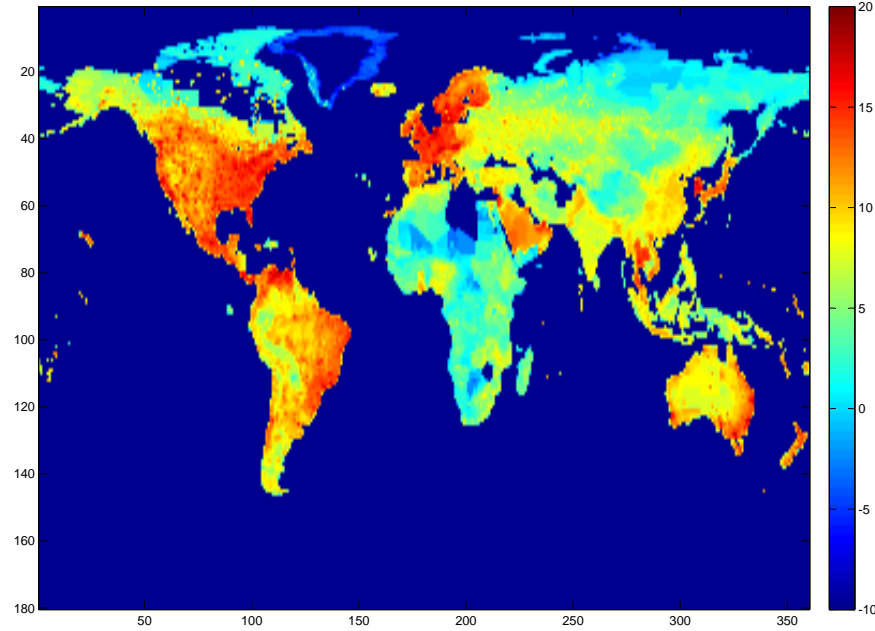

a. Population Density for $\psi=1.8$

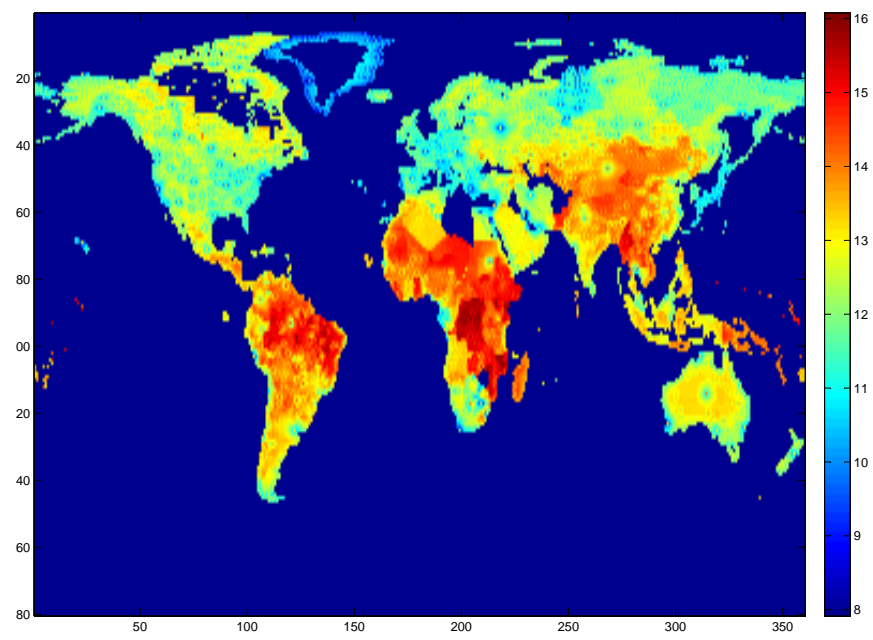

c. Amenities for $\psi=1.8: \bar{a}(r) \bar{L}_{t}(r)^{-\lambda}$

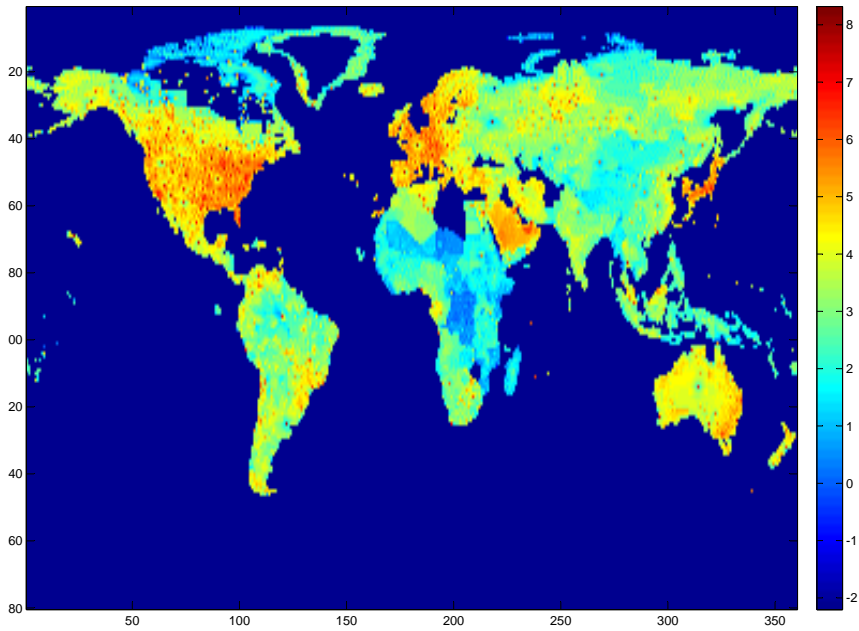

b. Productivity for $\psi=1.8$ : $\left[\tau_{t}(r) \bar{L}_{t}(r)^{\alpha}\right]^{\frac{1}{\theta}}$

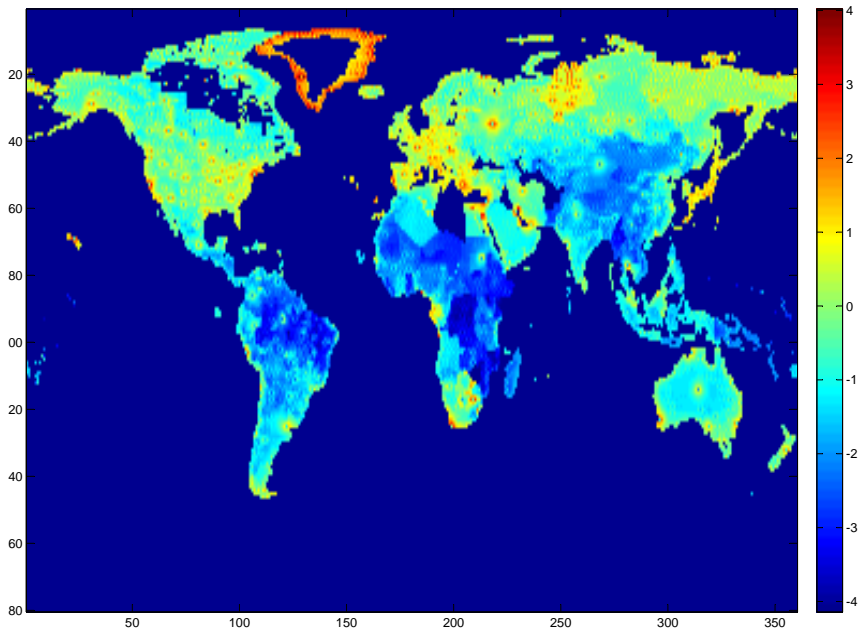

d. Real Income per Capita for $\psi=1.8$

When analyzing the average growth in real income per capita and utility, two differences become immediately apparent in Figure 5 when comparing the case of free migration $(\psi=1.8)$ to the case of no liberalization $(\psi=0)$. First, mobility increases the long-run growth rate of the economy as well as the average level of welfare, output 
Figure 7: Equilibrium with Free Migration (Period 600)

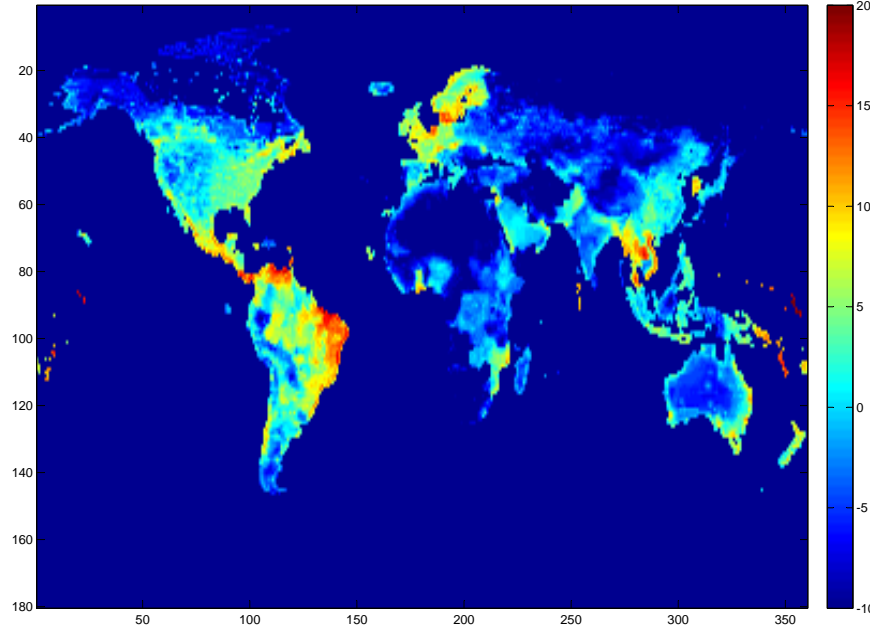

a. Population Density for $\psi=1.8$

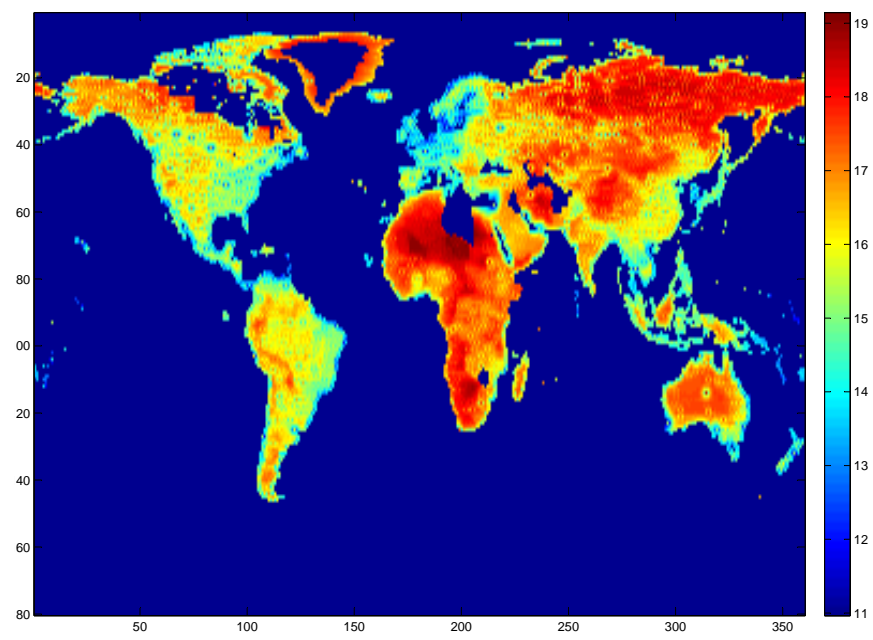

c. Amenities for $\psi=1.8: \bar{a}(r) \bar{L}_{t}(r)^{-\lambda}$

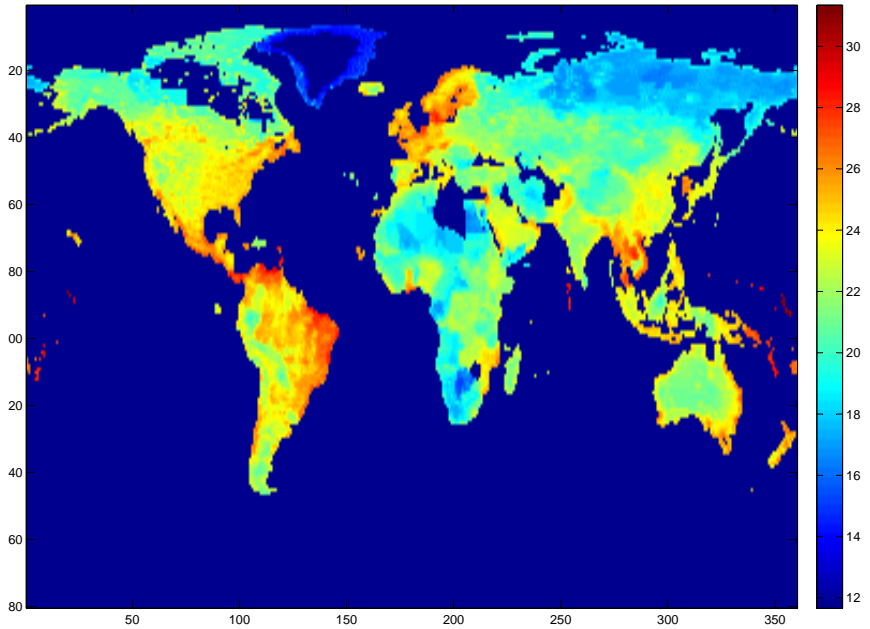

b. Productivity for $\psi=1.8$ : $\left[\tau_{t}(r) \bar{L}_{t}(r)^{\alpha}\right]^{\frac{1}{\theta}}$

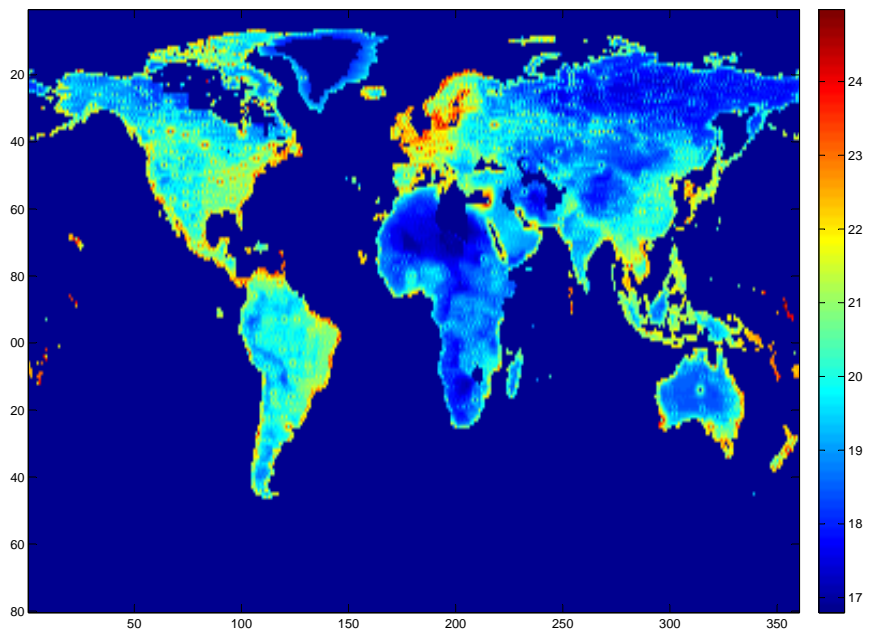

d. Real Income per Capita for $\psi=1.8$

and productivity in the world. Second, it is no longer the case that the growth rate in utility is lower in the long run than in the short run. Recall that in the case of no free migration the drop in the utility growth rate happened because initially the areas with high real GDP per capita growth were losing population. With free migration this is no longer the case.

\subsubsection{Partial liberalization}

We now present the changes in discounted real income and utility, as well as migration flows, that result from a variety of scenarios where we partially relax migration frictions. Figures 4 and 5 in the previous section already presented correlations as well as growth rates and levels of the different variables for the partial liberalization scenario, where we set $\psi=0.9$. As can be seen in Table 2, although this value of $\psi$ is right in the middle between keeping existing migration restrictions unchanged and free migration, it leads to reallocations of population that 
are closer to the free migration case. When we liberalize migration fully, $78.2 \%$ of the population changes country; the corresponding number when $\psi=0.9$ is a not much lower $65.0 \%$. As expected, the share of population that moves increases monotonically with the degree of liberalization.

In terms of welfare, migration frictions are tremendously important, particularly when we move closer to free migration 28 As shown in Table 2, a liberalization that implies that a quarter of the world population moves at impact $(\psi=0.3)$ yields a relatively modest increase in real output of $3.5 \%$, but a substantially larger increase in welfare of $71 \%$. A liberalization that leads to three quarters of the world population migrating at impact gives welfare gains of $298 \%$ and an increase in real income of $56.2 \%$. Full liberalization leads to welfare gains of $312 \%$, with only a relatively small increase in migration at impact of 4.3 percentage points 29

Three comments are in order when analyzing the numbers in Table 2 First, in general the gains in utility are much larger than the gains in real income, especially when migration becomes freer. Relaxing migration restrictions tends to relocate people to high-amenity countries. In the case of free migration this reallocation accounts for nearly $80 \%$ of the total welfare effect. Second, the mobility numbers presented in Table 2 include agents who move across countries at impact in period 1 only. Of course, as a result of migratory liberalization, the entire development path of the world economy changes dramatically as shown in Figures 4 to 7 . Third, real income numbers have been normalized in period 1 , so that they do not include the on-impact effects. As for welfare, Table 2 reports the present value of the population-weighted average utility levels after normalizing for the direct level effect of the change in migration restrictions ${ }^{30}$ This normalization implies that we disregard the direct welfare effects from the change in policy and take into account only the effects that result from the change in the equilibrium allocation that results from it. Otherwise, the gains from mobility would be even larger, as can be seen in the lower-right panel of Figure 5 where we present level effects without this normalization 31

Table 2: The Impact of Mobility Frictions

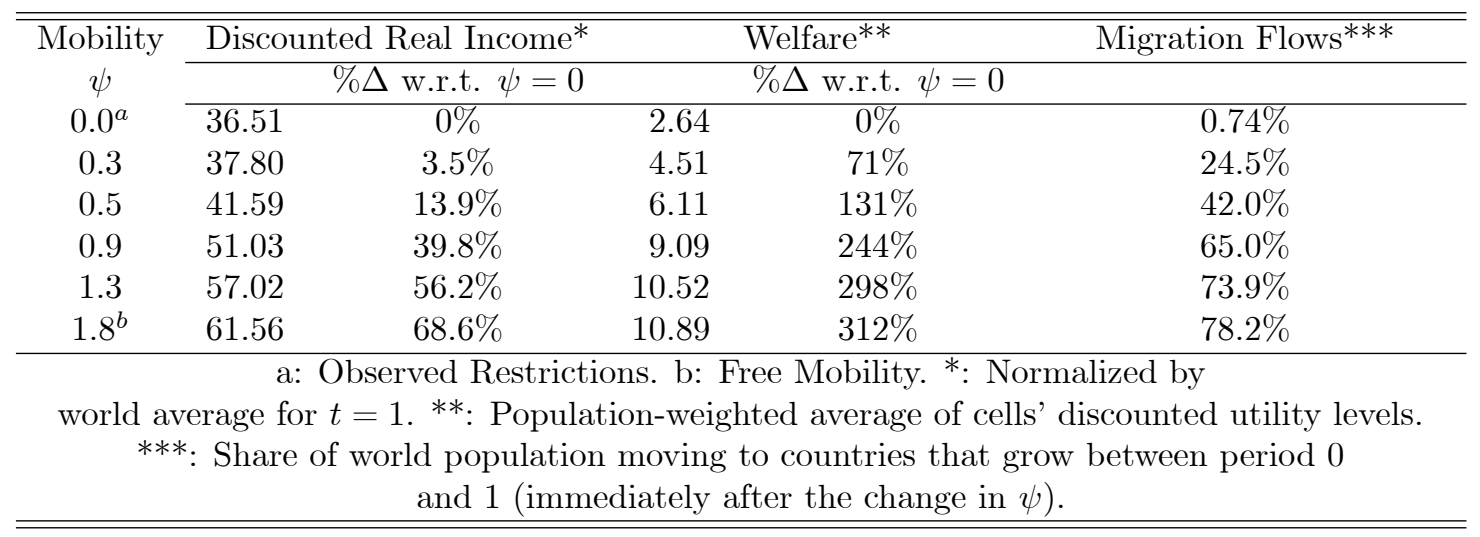

\footnotetext{
${ }^{28}$ These calculations assume that the economy is on its balanced growth path after period 600 . Note in Figure 5 that in all cases the balanced growth path growth rate remains strictly below the discount factor and so the discounted present value of income and utility is well defined.

${ }^{29}$ To the best of our knowledge, this is the first paper to analyze the global gains from mobility in an endogenous growth model with spatial heterogeneity, costly trade and amenities. Previous work, that focuses on models with capital accumulation, estimates long-run gains in income per capita of around 100\% (Klein and Ventura, 2007; Kennan, 2013). Though not directly comparable to the present discounted numbers reported in Table 2, we find larger effects. The main reason is that in our model mobility generates both a level and a long-run growth effect, whereas in these other models there is only a level effect as the economy moves to its new steady state.

${ }^{30}$ Namely, we calculate the population-weighted average of $\bar{u}_{t}^{W} e^{(1.8-\psi) \tilde{u}(c)}$.

${ }^{31}$ In that figure we just present $\bar{u}_{t}^{W}$.
} 


\subsection{Evaluating the Rise in Sea Levels}

The rise in the sea level is one of the major issues when assessing the effects of global warming. The thermal expansion of the oceans, the melting of many of the world's glaciers and the progressive depletion of the ice sheets in Greenland and Antarctica are major contributors to this rise. According to the International Panel on Climate Change (IPCC), sea levels are likely to rise by 0.5 to 1 meter by 2100 (Church et al., 2013). Over the longer horizon of a millennium, the expected loss of much of Greenland's ice sheet as a result of temperatures that are at least $2^{\circ}$ to $4^{\circ} \mathrm{C}$ higher would increase the sea level by 7 meters. The disproportionate location of the world's population in coastal areas bodes ill for many of its megacities, such as Mumbai, New York, Miami, Guangzhou and Osaka (Nicholls et al., 2008). More than 50\% of the U.S. population lives in locations prone to flooding, and every year, 1.2 million additional people are moving to those areas (Moser et al., 2014).

When evaluating the impact of the rise in sea levels, some papers focus on a simple accounting exercise based on current data. For example, Dasgupta et al. (2007) use detailed GIS data on current population and GDP to compute the impact of a rise in the sea level of between 1 and 5 meters. Although this gives some idea of what is at stake, these statements are unsatisfactory and simplistic for at least two reasons. First, much of the increase in the sea levels will happen in the future, rather than today, and there is no reason why the share of the population living in low-lying coastal areas, or the share of world GDP produced in those locations, will remain unchanged 100 or 500 years from now. Second, people can move in the face of rising sea levels. As this will lead to a different distribution of population across space, it will also lead to a different dynamic path for the economy.

Some papers partially address these issues by taking into account changing socio-economic conditions (see, e.g., Nicholls, 2004, Hinkel and Klein, 2009, and Anthoff et al., 2010). These papers typically rely on different socioeconomic scenarios developed by the IPCC in its Special Report on Emissions Scenarios (SRES). As an example, one scenario assumes rapid convergence in GDP per capita and in fertility across the different regions of the globe. However, since these scenarios only make predictions for large regions, going to the level of countries, let alone to the level of cells, is not easy. In most cases it is simply assumed that all cells in a given country experience identical growth patterns (Arnell et al., 2004). This is unfortunate, since future changes within countries are likely to be key when assessing the damage of coastal flooding. In addition, these models are unable to address how the economy will adjust when parts of it get flooded.

Our model is well equipped to address these issues. By proposing a general equilibrium spatial growth model that incorporates trade, migration and innovation, our framework endogenously generates how the spatial distribution of population, GDP and amenities changes over time, both within and across countries. Our results suggest that, depending on the assumption we make about migration, the negative impact of flooding on the economy can change dramatically. For example, if the sea level were to rise by 6 meters in 2100 and we kept migratory restrictions unchanged, $15 \%$ of the world's population would get flooded. This figure would increase to $54 \%$ under free mobility. In spite of making matters worse in terms of how many people end up under water, we would expect greater mobility to have an advantage in the aftermath of flooding, as it should facilitate relocating people. However, in a decentralized economy, there is no guarantee that people will coordinate on moving to the "right" locations, so that more mobility need not improve the welfare impact of relocation.

In what follows we first analyze the static cost of rising sea levels, depending on when the rise will occur. We then look at the dynamic effects of a rise in sea levels, with a particular focus on the welfare effects. In all of the exercises we assume an increase in sea levels by 6 meters, but we will also briefly compare our findings to a less dramatic 1 meter rise in sea levels. The information on what areas would get flooded comes from maps generated 
by the Center for Remote Censoring of Ice Sheets (Cresis). To give a preliminary idea of the impact of flooding, Figure 8 shows today's population in areas that would be flooded if sea levels rose by 6 meters, and Figure 9 provides close-ups for different regions.

Figure 8: Population Flooded with Sea Level Rise of 6 Meters (Data of 2000)

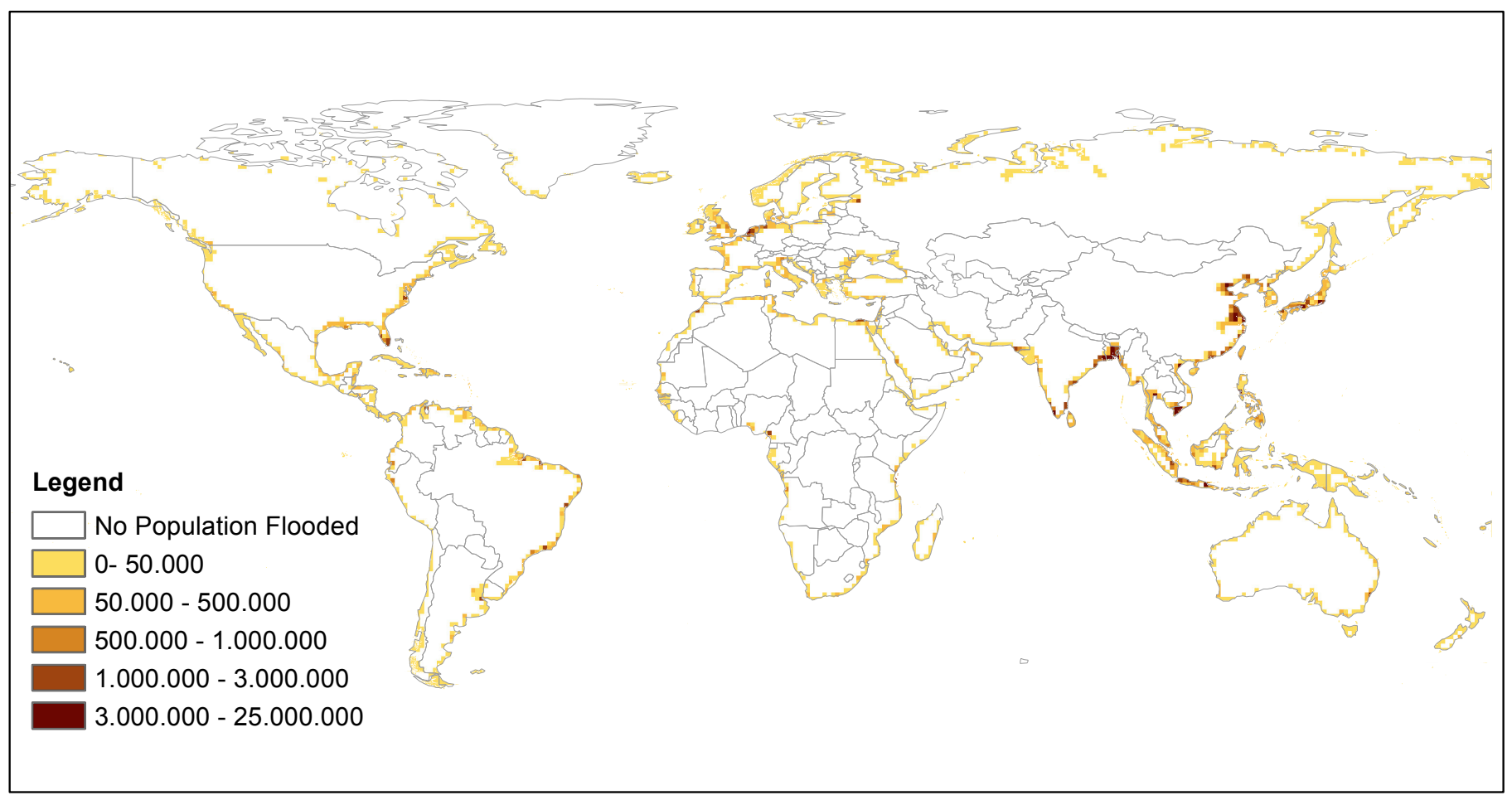

\subsubsection{Timing of the rise in sea levels}

In this section we analyze the static cost of a rise in the sea level, depending on when that rise occurs and depending on which assumptions we make on mobility. Our starting point is the exercises of the previous subsection, where we analyzed the evolution of the spatial distribution of population and aggregate growth under different mobility assumptions. We then shock the world with a rise in the sea level by 6 meters and analyze how much of the population, how much of the amenities and how much of the productivity gets lost upon impact. Since the spatial distribution of population and production differs across time and depends on the mobility assumptions, the answer to that question will depend on when the sea level rises and on how mobile the population is.

Table 3 (Panel A) reports the results for a world in which mobility restrictions are kept unchanged. Hence, we start off with the exercise in subsection 5.1, and we let the sea level rise by 6 meters in either period 1, period 100 or period 500. Upon impact, without giving the economy a chance to react, we compute the percentage of population flooded, the percentage of land rents lost, the percentage of technology lost and the percentage of amenities lost. This gives us a sense of the economic magnitude of the shock, depending on when it happens. Before discussing the results, it is important to define some terms. As before, technology is defined as $\left[\tau_{t}(r) \bar{L}_{t}(r)^{\alpha}\right]^{1 / \theta}$ and amenities are defined as $\bar{a}(r) \bar{L}_{t}(r)^{-\lambda}$. When discussing the percentage of technology lost, we weight technology either by 
Figure 9: Population Flooded with Sea Level Rise of 6 Meters (Different Regions, Data of 2000)

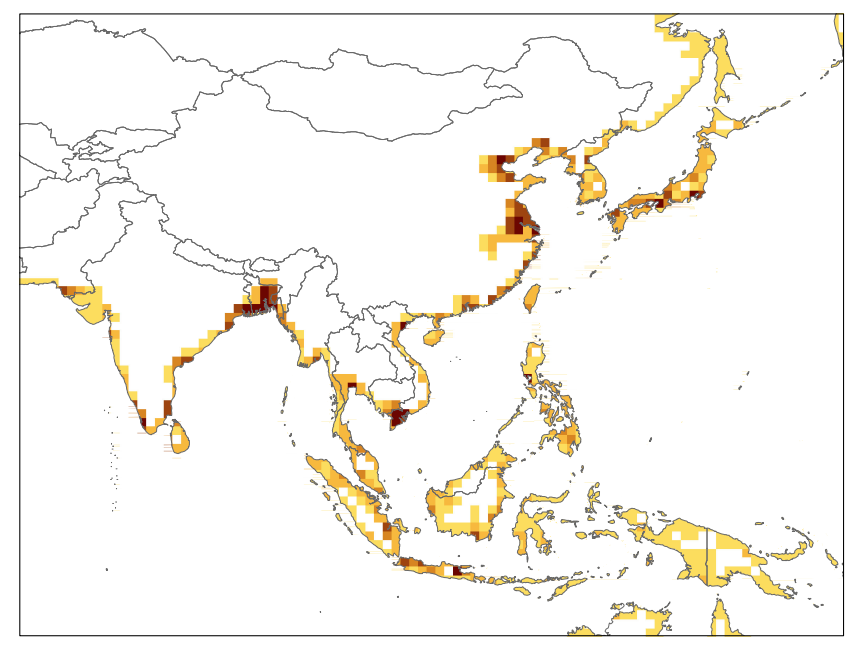

a. Asia

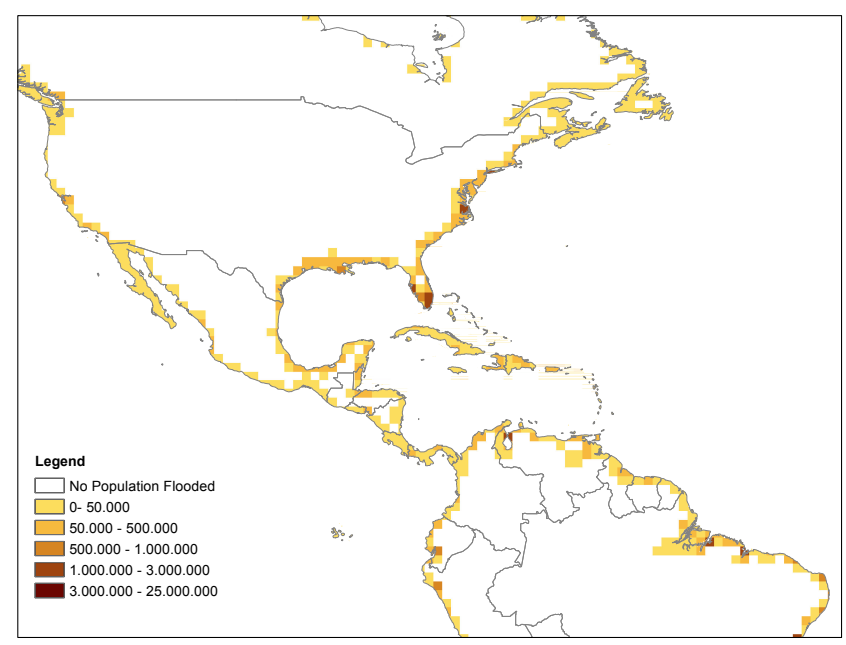

c. North America

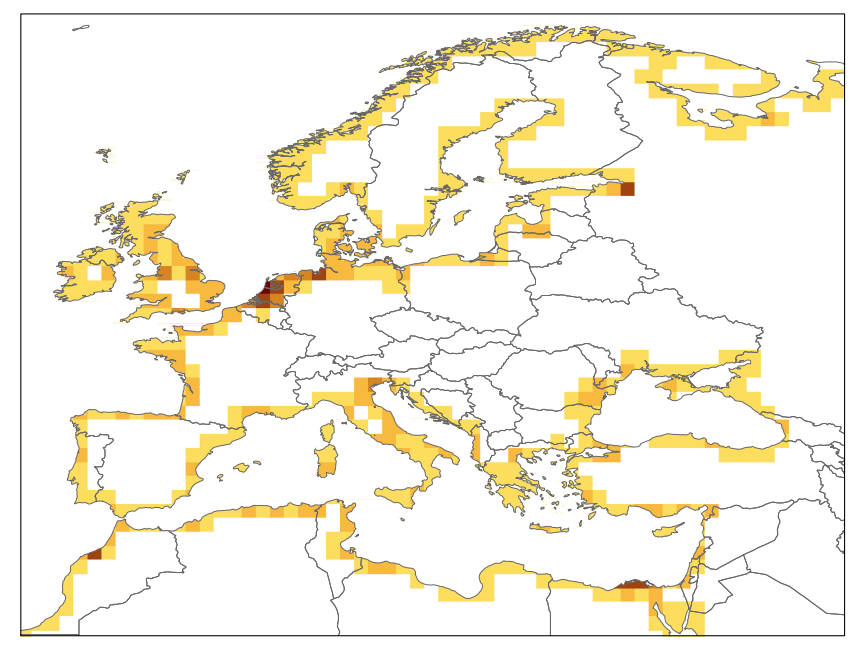

b. Europe

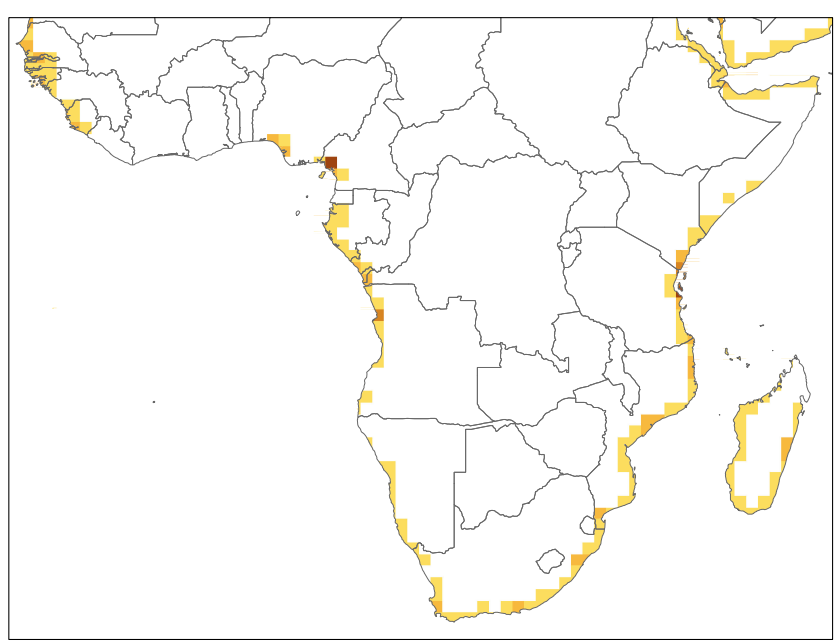

d. Africa

land or by population. We do the same for amenities.

A number of findings stand out. First, the percentage of population flooded is greater than the percentage of land flooded. Whereas $1 \%$ of land gets flooded, $6.6 \%$ of population gets flooded if the sea level rises in 2000. Second, over time this difference first increases, and then decreases. If the rise in the sea levels were to occur in $2100,7.5 \%$ of the population would be under water. By 2500, this percentage decreases to $5.7 \%$. Third, the percentage of technology lost is also greater than the share of land flooded, even when technology is weighted by land, rather than by population. This reflects the fact that coastal areas are more productive than non-coastal areas. Of course, when we weight technology by population, this difference is reinforced, given the disproportionate share of people living on the coast. Fourth, the percentage of amenities lost is initially larger than the percentage of land lost, but this gets reversed over time. This reflects partly the increase in population in coastal areas, which pushes 
Table 3: The Effect of a Rise in Sea Levels by 6 Meters

\begin{tabular}{lccc}
\hline \hline & Period 1 & Period 100 & Period 500 \\
\hline A. Keeping mobility restrictions unchanged & $(\psi=0)$ & & \\
Percentage land flooded & 1.0 & 1.0 & 1.0 \\
Percentage population flooded & 6.6 & 7.5 & 5.7 \\
Percentage land rents lost & 6.3 & 8.0 & 6.2 \\
Percentage technology lost (land weighted) & 2.4 & 2.5 & 2.6 \\
Percentage technology lost (population weighted) & 9.5 & 10.5 & 5.7 \\
Percentage amenities lost (land weighted) & 2.2 & 1.6 & 0.8 \\
Percentage amenities lost (population weighted) & 8.8 & 9.6 & 9.6 \\
\hline B. Free mobility ( $\psi$ (1.8) & & & \\
Percentage land flooded & 1.0 & 1.0 & 1.0 \\
Percentage population flooded & 11.2 & 14.9 & 81.4 \\
Percentage land rents lost & 8.4 & 13.7 & 89.1 \\
Percentage technology lost (land weighted) & 2.4 & 3.0 & 6.0 \\
Percentage technology lost (population weighted) & 11.6 & 19.4 & 97.4 \\
Percentage amenities lost (land weighted) & 1.2 & 0.9 & 0.5 \\
Percentage amenities lost (population weighted) & 11.9 & 18.0 & 80.9 \\
\hline C. Partial mobility ( $\psi$ (1.9) & & & \\
Percentage land flooded & 1.0 & 1.0 & 1.0 \\
Percentage population flooded & 9.1 & 12.4 & 33.8 \\
Percentage land rents lost & 7.2 & 10.0 & 41.6 \\
Percentage technology lost (land weighted) & 2.4 & 2.8 & 4.6 \\
Percentage technology lost (population weighted) & 10.0 & 15.3 & 65.5 \\
Percentage amenities lost (land weighted) & 1.8 & 1.4 & 0.8 \\
Percentage amenities lost (population weighted) & 10.7 & 15.2 & 56.9 \\
\hline
\end{tabular}

down the value of amenities, but there is also another effect: the reallocation of people within countries tends to move people from low-amenity to high-amenity places. This explains why the relation between the percentage of amenities lost and the percentage of population flooded may not be monotone.

Table 3 (Panel B) redoes the exercise for free migration. The most striking finding, already discussed in the previous section, is that under free mobility, a significantly greater share of people move to low-lying coastal areas. Free mobility moves people toward high-density, high-productivity locations, which are disproportionately located in coastal regions. This move gets reinforced over time, because of the positive effect of density on productivity. If the rise in sea levels were to happen in 2500 , as much as $81.4 \%$ of the world population would get flooded. Not surprisingly, the percentage of technology and amenities lost, when weighted by population, is mostly driven by the population dynamics. When weighted by land, rather than by population, the percentage of technology lost increases over time (reflecting the positive relation between population and productivity), whereas the percentage of amenities lost decreases over time (reflecting the negative relation between population and amenities). Panel C in Table 3 focuses on the in-between case of partial liberalization of mobility restrictions. It illustrates that as we relax migration restrictions, we tend to move monotonically between the two cases described in more detail above.

Above we described the economic impact of flooding by analyzing the shares of population, land rents, amenities and technology lost. Another way of looking at the same phenomenon is by comparing the distribution of, say, land rents under different scenarios. Figure 10 plots the distribution of the log of land rents (weighted by area) under different mobility assumptions for period 1 . As can be seen, liberalizing migration restrictions increases the 
dispersion of land rents. As expected, more mobility implies that more desirable locations become more crowded and less desired locations less crowded. This pushes up land rents in attractive places, with the opposite happening in less attractive places. Figure $10 \mathrm{~b}$ compares flooded vs. non-flooded areas. There the result that stands out is that flooded areas tend to have higher land rents than non-flooded areas independently of the migration scenario we analyze. This is consistent with the share of rents lost to flooding being larger than the share of land lost to flooding. Coastal areas are economically valuable due to high amenities and low transport costs and are, obviously, the ones that are prone to be flooded.

Figure 10: Rent Distributions

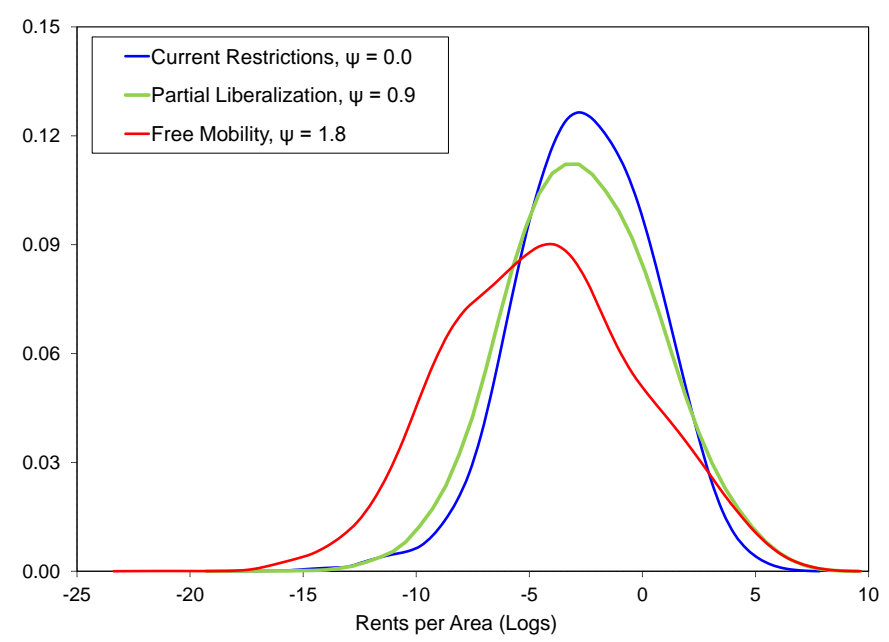

a. Different Mobility Scenarios

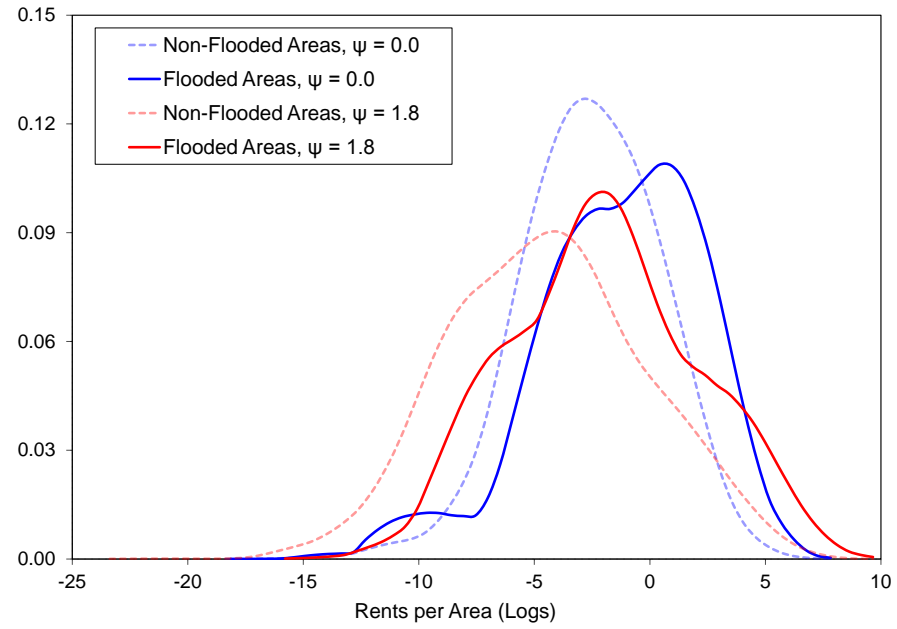

b. Flooded vs. Non-Flooded Areas

\subsubsection{Dynamic effects of the rise in sea levels}

We now turn to analyzing the dynamic effects of coastal flooding by taking into account how the economy reacts to a rise in sea levels. In this exercise, the sea level rises by 6 meters between period 0 and period 1 . As a result, people move to other areas, and the economy gets on a different dynamic path. To assess the welfare costs of flooding, Table 4 reports the present discounted value of real income per capita and of utility for different values of $\psi$. The evolution of the correlation between GDP per capita, population density and productivity, as well as the paths of growth rates and levels, was already included in Figures 4 and 5 Also recall that $\psi$ is negatively associated with mobility restrictions: $\psi=1.8$ corresponds to free migration and $\psi=0$ corresponds to the currently observed restrictions as measured by the data on subjective well-being.

As we know from Table 2 mobility increases real income per capita and utility. When comparing a situation where we leave migratory restrictions unchanged $(\psi=0)$ to one where we have free migration $(\psi=1.8)$, the present discounted value of utility, our measure of welfare, is multiplied by more than four. This is true whether we assume a world with flooding or a world without flooding. This increase in welfare is much larger than the corresponding increase in the present value of income that grows by $69 \%$. Since the only difference between real income and utility is the level of amenities, this implies that an important advantage of mobility is that it moves people from low-amenity to high-amenity places. So the main results on the gains from migration remain in place independently of whether the world experiences flooding. 
Table 4: Dynamic Effects of Coastal Flooding (6 Meters)

\begin{tabular}{|c|c|c|c|c|c|c|}
\hline \multirow{2}{*}{$\begin{array}{c}\text { Mobility } \\
\psi\end{array}$} & \multicolumn{3}{|c|}{ Discounted Present Value of Real Income* } & \multicolumn{3}{|c|}{ Welfare** } \\
\hline & No Flooding & Flooding & Ratio (NF/F) & No Flooding & Flooding & Ratio $(\mathrm{NF} / \mathrm{F})$ \\
\hline $0.0^{a}$ & 36.51 & 35.21 & 1.037 & 2.64 & 2.44 & 1.082 \\
\hline 0.3 & 37.80 & 36.77 & 1.028 & 4.51 & 4.18 & 1.079 \\
\hline 0.5 & 41.59 & 40.72 & 1.021 & 6.11 & 5.68 & 1.075 \\
\hline 0.9 & 51.03 & 50.24 & 1.016 & 9.09 & 8.48 & 1.072 \\
\hline 1.3 & 57.02 & 55.69 & 1.024 & 10.52 & 9.77 & 1.076 \\
\hline $1.8^{b}$ & 61.56 & 59.39 & 1.037 & 10.89 & 10.10 & 1.078 \\
\hline & $\begin{array}{l}*: \mathrm{No} \\
* *: \mathrm{Pc}\end{array}$ & a: Obse & $\begin{array}{l}\text { Restrictions. } \\
\text { d average GDP } \\
\text { d average of ce }\end{array}$ & $\begin{array}{l}\text { Free Mobility } \\
\text { ithout floodin } \\
\text { 'discounted } u\end{array}$ & $\begin{array}{l}\text { for } t=1 \text {. } \\
\text { lity levels. }\end{array}$ & \\
\hline
\end{tabular}

We now turn to comparing real income and welfare in a world without flooding to one in a world with flooding. Several results stand out in Table 4 First, flooding always leads to a loss in both real income and welfare. In the case of real income, the drop ranges from $1.6 \%$ to $3.7 \%$ depending on our assumptions on mobility, whereas in the case of utility the drop ranges from $7.2 \%$ to $8.2 \%$. Second, the impact of flooding on the present value of welfare has only a weak, and non-monotonic, relation with mobility. When mobility restrictions are as in the current world economy, the losses are $8.2 \%$. These losses decline to $7.2 \%$ for intermediate levels of migration restrictions $(\psi=0.9)$, and then increases again, to $7.8 \%$, in a world with free migration. We see a similar U-shaped pattern for income per capita.

The finding that flooding has similar effects, whether we maintain existing frictions or allow for free migration, may come as a surprise. In principle, one would expect mobility to mitigate the negative welfare effect of flooding. In fact, in Desmet and Rossi-Hansberg (2014b) we argued that adaptation through mobility can help reduce the economic cost of global warming. There are three reasons why here we find that the benign effect of mobility is eroded. First, when we analyze the effect of liberalizing migration, we are exclusively talking about changing mobility across countries, since in all exercises there is always free mobility within countries. Hence, since mobility within countries should be enough to absorb shocks due to flooding (with the exception of maybe some small islands in the Pacific Ocean), we should not expect mobility across countries to make a huge difference through this direct effect. Second, since greater mobility across countries causes more people to locate in low-lying coastal areas, the impact of flooding is greater, since it distorts the equilibrium more. That is, although greater mobility makes moving easier, it also requires more people to move. Third, and maybe most important, different migration policies put the economy on different dynamic paths, so that a priori there is no reason why greater mobility should mitigate the impact of flooding.

To clarify this last point, assume that in the face of a 6-meter rise in the sea level that causes the flooding of $1 \%$ of the land's surface, the planner could push everyone some miles more inland to higher ground. Abstracting from the moving costs, we would not expect a major impact. However, in a decentralized economy people may move to already established locations far-off rather than simply a little bit more inland. This behavior is optimal for the individuals but would not be the choice of a central planner as it decreases overall growth and the level of income. In fact, absent this conflict, the paths with and without flooding in Figure 5 should be virtually identical, and the cost negligible. However, by giving individuals a wider set of options, more mobility makes it easier for them to move to farther off places, thus exacerbating the difference between what a planner would do and what 
individuals actually do. Furthermore, recall that with higher mobility more people concentrate in low-lying coastal lands, so that more people need to move when sea levels rise. Hence, the scope of the possible discrepancy between the decentralized economy and the planner's solution increases. This reasoning implies that, particularly in the long run, the losses from flooding increase as we reduce migration restrictions.

Rather than looking at summary statistics, Figure 5 shows the entire dynamic path of productivity, real GDP and utility, comparing flooding and no flooding under different mobility restrictions ${ }^{32}$ Essentially these figures show that flooding causes a negative shock to the growth rates of productivity, real GDP and utility. This effect is larger in the medium-term and in the case of free migration. The second row of Figure 5 presents the accumulated level effect of flooding, which is particularly large for the case of free migration. To sharpen the comparison between no flooding and flooding, Figure 11 shows the relative dynamic paths of real GDP per capita and utility. In a world where we keep migratory restrictions unchanged, the immediate impact of flooding on real GDP and utility is negative, but the long-run impact is positive. In contrast, in a world with free mobility, the welfare effect of flooding is always negative, while for output it is negative in the short run and in the long run, with a short interval after 200 years where it reverses sign. As is evident from the figure, flooding has a potentially large impact on real GDP and welfare and interacts dramatically with migration restrictions. This is particularly true in the long run. The level of migration restrictions determines ultimately the effect of flooding on the steady-state growth rate and, therefore, its long-run impact. Figure 11 shows that the implications on the present value of real output and welfare in Table 4 are the result of balancing short-term and long-term changes: migration restrictions increase the losses from flooding in the short run, but decrease them in the long run, explaining why in present value terms the differences are small. Furthermore, Figure 11 underscores the argument that relatively small geographic shocks like flooding can have a large impact on long-term outcomes by changing the development path of the economy.

Figure 11: No Flooding vs Flooding (Levels)
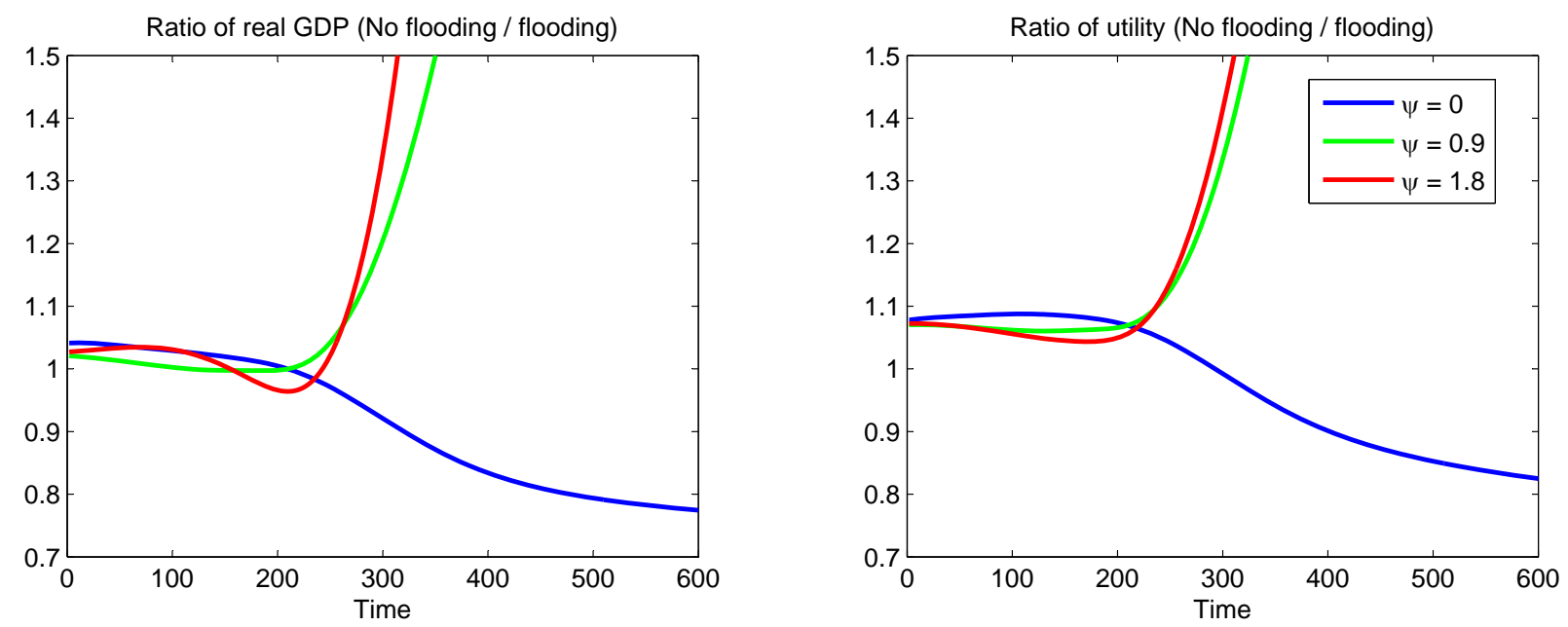

According to the IPCC, sea levels are likely to rise by 0.5 to 1 meter by 2100 (Church et al., 2013). Since a 6-meter rise in sea levels is an extreme and far-off prospect, we are also interested in understanding how the effects of a sea level rise scale down as we consider a 1-meter increase. As expected, the effects are smaller, but less than proportionally so. Whereas a 6 -meter rise today is predicted to lead to $1 \%$ of land lost, $6.6 \%$ of population

\footnotetext{
${ }^{32}$ For videos that show the evolution over space of the effect of flooding, see the Online Appendix
} 
flooded and $6.3 \%$ of land rents lost, the corresponding figures for a 1-meter rise are $0.4 \%, 1.6 \%$ and $1.9 \%$. Under free mobility, the differences between the different levels of flooding are not as big. A 6-meter rise today would imply $11.2 \%$ of population flooded and $8.4 \%$ of land rents lost, whereas the corresponding figures with a 1-meter rise are $5.5 \%$ and $4.2 \% 33$ As for the dynamic effects of coastal flooding, a 1-meter rise does not change the results qualitatively. As before, the drop in the present value of welfare varies little with the degree of mobility, and is around $3.5 \%$ to $4 \%$ Hence, a six-time smaller rise in sea levels, that floods an area that is only 40 percent its size, with about a fourth of the population, generates losses that are about half the size.

\section{Conclusion}

The complex interaction between geography and economic development is at the core of a wide variety of important economic phenomena. In our analysis we have underscored the fact that the world is interconnected through trade, technology diffusion, and migration. We have conducted our analysis at a fine geographic detail, enabling us to incorporate the significant heterogeneity across regions in amenities and productivity. This framework and quantification have allowed us to evaluate the impact of migration frictions and to assess the effect of a potentially important geographic shock like coasting flooding.

Our results highlight the complexity of the impact of geographic changes as well as the importance of their interaction with factor mobility. We found that relaxing migration restrictions can lead to very large welfare gains, but that the world economy will concentrate in very different sets of nations depending on migratory frictions. This inequity in the cross-country economic implications of relaxing migration frictions will undoubtedly lead to political disagreements about their implementation. For example, the developed economies today will guarantee their future economic superiority only in scenarios where the world relaxes migration restrictions. We have abstracted from these political economy considerations, but they are extremely important.

Even though our framework incorporates a large set of forces in a rather large general equilibrium exercise, we had to make the choice of leaving some important forces aside. Perhaps the most relevant one is the ability of the world economy to accumulate a factor of production like capital. In our framework, only technology can be accumulated over time, not capital. In our view, the ability of regions to invest in technology substitutes partially for the lack of capital accumulation, but clearly not fully. We also abstracted from local investments in amenities. In our framework, amenities vary only through changes in congestion as a result of in or out migration. Finally, we also abstracted from individual heterogeneity in education or skills. Given our long-term focus, we view educational heterogeneity as part of the local technology of regions. It is obviously the case that individuals can migrate with their human capital, and we ignore this. However, the human capital embedded in migrants lasts mostly for only one generation. Ultimately, migrants and, more important their descendants, will obtain the local education that is part of the local technology of a given region.

\footnotetext{
${ }^{33}$ Table 7 in Appendix A.4 gives all the details.

${ }^{34}$ See Table 8 in Appendix A.4.
} 


\section{Appendix A: Density-Income Correlation and Subjective Well-Being}

\section{A.1 Correlation of Density and Income}

In our theory, the presence of both static and dynamic agglomeration economies, together with the role played by amenities, implies that the correlation between density and income per capita is relatively low (and possibly negative) when income per capita is low, and relatively high when income per capita is high. Two forces drive this result. On the one hand, the standard positive correlation between density and per capita income, due to static agglomeration economies, is stronger in high-productivity places that benefit from greater dynamic agglomeration economies. This explains why the correlation between density and per capita income is increasing in per capita income. On the other hand, free mobility means that high-amenity places tend to have both high population density and low per capita income. This explains why in relatively low per capita income locations, where dynamic agglomeration economies are weak, the correlation between density and per capita income might be negative.

To see whether these findings hold up in the data, we do two exercises. First, we compute the correlation between population density and income per worker across U.S. zip codes. We split zip codes into different groups: those with income per capita (worker) below the median and those with income per capita (worker) above the median. The theory predicts a higher correlation between density and income per capita for the latter group and possibly a negative correlation for the former group. We also consider a finer split-up into four different groups by income per capita quartile. In that case, we would expect the correlation to increase when we go from lower income per capita quartiles to higher income per capita quartiles.

Table 5 reports the results. Data on population, mean income per worker and geographic area come from the 2000 U.S. Census and from the American Community Survey 5-year estimates. The geographic unit of observation are ZIP Code Tabulation Areas (ZCTAs). We use two different definitions of income: "earnings" correspond essentially to labor income, whereas "total income" also includes capital income. As predicted by the theory, Panel A shows that the correlation between population density and earnings per full-time worker, both measured in logs, increases from 0.12 for ZCATs with below-median earnings per full-time worker to 0.31 for ZCATs above the median. When we focus on total income in Panel B, the results are similar, but now the correlation between population density and income per full-time worker is negative for ZCATs below the median. In particular, the correlation increases from -0.06 for those ZCATs below the median to 0.36 for those above the median. All these correlations are statistically different from zero at the $1 \%$ level, and in both cases, the increase in the correlation is statistically significant at the $1 \%$ level. When we compare different quartiles, rather than below and above the median, the results continue to be consistent with the theory: the correlations between density and income are greater for higher-income quartiles.

Second, we explore whether the relation between density and income is stronger in richer areas by analyzing how that relation at the zip code level changes with the relative income of the Core Based Statistical Area (CBSA) the zip code pertains to. To analyze this, we start by running separate regressions for each CBSA of payroll per employee on employee density at the zip code level. Using data of 2010 from the ZIP Code Business Patterns, this yields 653 coefficients of employee density, one for each CBSA. Figure 12 then plots these 653 coefficients against

the relative income of the CBSA. As expected, as the relative income of the CBSA increases, the effect of employee density on payroll per employee increases.

Rather than running separate regressions for each CBSA, we can also run a single regression with an interaction 
Table 5: Density and Income

\begin{tabular}{|c|c|c|c|c|c|c|}
\hline \multicolumn{7}{|c|}{ 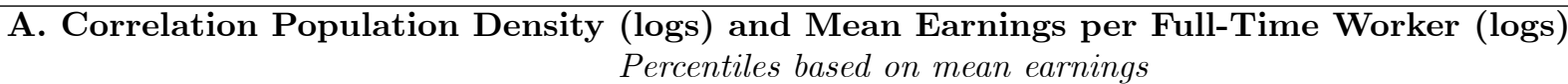 } \\
\hline Year & $<25$ th & $25-50$ th & 50th-75th & $>75$ th & $<$ Median & $\geq$ Median \\
\hline 2000 & $0.0549^{* * *}$ & $0.0884^{* * *}$ & $0.1293^{* * *}$ & $0.2199 * * *$ & $0.1216^{* * *}$ & $0.3128^{* * *}$ \\
\hline $2007-2011$ & $-0.0237^{*}$ & $0.0428 * * *$ & $0.1048^{* * *}$ & $0.2727^{* * *}$ & $0.0222^{* * *}$ & $0.3546^{* * *}$ \\
\hline \multicolumn{7}{|c|}{ B. Correlation Population Density (logs) and Per Capita Income (logs) } \\
\hline Year & $<25$ th & 25-50th & 50th-75th & $>75$ th & $<$ Median & $\geq$ Median \\
\hline 2000 & $-0.1001^{* * *}$ & $0.0495^{* * *}$ & $0.1499^{* * *}$ & $0.2248^{* * *}$ & $-0.0609^{* * *}$ & $0.3589^{* * *}$ \\
\hline 2007-2011 & $-0.0930^{* * *}$ & 0.0175 & $0.0733^{* * *}$ & $0.2420^{* * *}$ & $-0.0781^{* * *}$ & $0.3234^{* * *}$ \\
\hline
\end{tabular}

Figure 12: Payroll per Employee and Employee Density

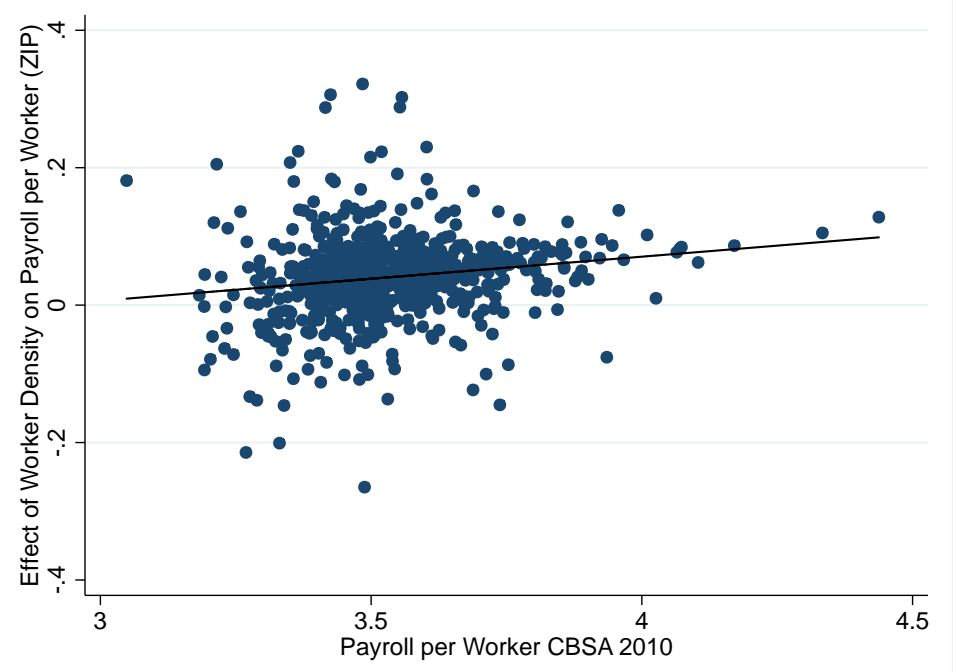

term of density at the zip code level with payroll per employee at the CBSA level:

$$
y(z i p)=a_{0}+a_{1} d(z i p)+a_{2} d(z i p) y(c b s a)+\varepsilon(z i p)
$$

where $y(z i p)$ is payroll per employee at the zip code level, $y(z i p)$ is payroll per employee at the CBSA level, and $d(z i p)$ is employee density at the zip code level. The theory predicts that $a_{1}$ may be negative and that $a_{2}$ is positive. Consistent with this, when using data from 2010, we find $a_{1}=-0.180$ and $a_{2}=0.063$, and both are statistically significant at the $1 \%$ level. Using data from 2000 yields similar results: $a_{1}=-0.075$ and $a_{2}=0.039$, and once again both are statistically significant at the $1 \%$ level.

\section{A.2 Correlations of Amenities and Quality of Life}

Our goal is to compute correlations between our estimated measures of fundamental amenities and different exogenous measures of a location's quality of life. The different variables on quality of life, related to distance to 
water, elevation, precipitation, temperature and vegetation, come from G-Econ $4.04^{35}$ The results are reported in Table 6 .

Table 6: Correlations between Estimated Amenities and Different Measures of Quality of Life

\begin{tabular}{|c|c|c|c|c|c|}
\hline \multicolumn{6}{|c|}{ Correlations with Estimated Amenities (logs) } \\
\hline & $(1)$ & $(2)$ & $(3)$ & $(4)$ & $(5)$ \\
\hline & All cells & U.S. & $\begin{array}{c}\text { One cell } \\
\text { per country }\end{array}$ & $\begin{array}{c}\text { Placebo } \\
\text { of }(1)\end{array}$ & $\begin{array}{c}\text { Placebo } \\
\text { of }(3)\end{array}$ \\
\hline \multicolumn{6}{|l|}{ A. Water } \\
\hline Distance to ocean & $-0.2918^{* * *}$ & $-0.6300^{* * *}$ & $-0.2052^{* * *}$ & $-0.0983^{* * *}$ & $0.1911^{* * *}$ \\
\hline Distance to water & $-0.4546^{* * *}$ & $-0.2602^{* * *}$ & $-0.2388 * * *$ & $-0.3329 * * *$ & 0.2285 \\
\hline Water $<50 \mathrm{~km}$ & $0.2198 * * *$ & $0.1286^{* * *}$ & $0.1232^{* *}$ & $0.1064^{* * *}$ & $-0.1363^{* *}$ \\
\hline \multicolumn{6}{|l|}{ B. Elevation } \\
\hline Level & $-0.4152^{* * *}$ & $-0.1493^{* * *}$ & $-0.2816^{* * *}$ & $-0.2793^{* * *}$ & $0.1283^{* *}$ \\
\hline Standard deviation & $-0.4599 * * *$ & $-0.2573^{* * *}$ & $-0.3099 * * *$ & $-0.3285^{* * *}$ & $0.1121^{*}$ \\
\hline \multicolumn{6}{|l|}{ C. Precipitation } \\
\hline Average & $0.4176^{* * *}$ & $0.08643^{* * *}$ & $0.3851^{* * *}$ & $0.3185^{* * *}$ & $0.1830^{* * *}$ \\
\hline Maximum & $0.4408^{* * *}$ & $0.1068^{* * *}$ & $0.3128^{* * *}$ & $0.4286^{* * *}$ & $0.3200^{* * *}$ \\
\hline Minimum & $0.2035^{* * *}$ & $0.2136^{* * *}$ & $0.2108^{* * *}$ & -0.0096 & $-0.1965^{* *}$ \\
\hline Standard deviation & $0.4160^{* * *}$ & 0.0212 & $0.2746^{* * *}$ & $0.4715^{* * *}$ & $0.4535^{* * *}$ \\
\hline \multicolumn{6}{|l|}{ D. Temperature } \\
\hline Average & $0.6241^{* * *}$ & $0.6928 * * *$ & $0.3087^{* * *}$ & $0.6914^{* * *}$ & $0.5692^{* * *}$ \\
\hline Maximum & $0.5447^{* * *}$ & $0.7388^{* * *}$ & $0.1276^{* * *}$ & $0.6589 * * *$ & $0.4635^{* * *}$ \\
\hline Minimum & $0.6128 * * *$ & $0.6060 * * *$ & $0.2931^{* * *}$ & $0.6565 * * *$ & $0.5389 * * *$ \\
\hline Standard deviation & $-0.5587^{* * *}$ & $-0.3112^{* * *}$ & $-0.3313^{* * *}$ & $-0.5539 * * *$ & $-0.3679 * * *$ \\
\hline \multicolumn{6}{|l|}{ E. Vegetation } \\
\hline Desert, ice or tundra & $-0.3201^{* * *}$ & $-0.3993^{* * *}$ & $-0.1827^{* * *}$ & $-0.2440^{* * *}$ & $-0.1291^{*}$ \\
\hline
\end{tabular}

Column (1) are correlations based on all $1^{\circ}$ by $1^{\circ}$ cells of the world. These correlations suggest that people prefer to live close to water, dislike high elevations and rough terrain, like precipitation but not constantly, prefer warm and stable temperatures, and dislike deserts, tundras and ice-covered areas. It is reassuring that these correlations are largely consistent with those found in the literature. For example, Albouy et al. (2014) provide evidence that Americans have a mild preference for precipitation and a strong preference to live close to water, and Morris and Ortalo-Magné (2007) find that there is a positive correlation between quality of life and average temperature and precipitation. One thing that may come as a surprise is that people not only prefer higher average temperatures, they also like higher maximum temperatures. This result is driven by the imposed linearity: once we allow for a quadratic relation between our measure of amenities and maximum temperatures, we find an optimal maximum temperature of 27.4 degrees Celsius.

The fact that our correlations are overall in line with what we would expect suggests that our methodology of identifying amenities by using data on subjective well-being is reasonable. To further confirm this, we compare our results to what these correlations would look like within the U.S. Because utility is the same across all cells within a country, the correlations within the U.S. do not depend on our use of data on subjective well-being. Hence, if the

\footnotetext{
${ }^{35}$ All variables come directly from G-Econ 4.0, but a couple require some further manipulation. In particular, the "distance to water" measure is defined as the minimum distance to a major navigable lake, a navigable rivers or an ice-free ocean; and the different vegetation categories in G-Econ can be found at http://data.giss.nasa.gov/landuse/vegeem.html
} 
results for the U.S. are similar to those of the world, this is further evidence in favor of our methodology. Column (2) confirms that this is indeed the case.

Another possible worry is that the correlations across all cells of the world are driven by a few large countries. If so, what we observe in Column (1) may mostly reflect within-country variation, and this might explain why Columns (1) and (2) look similar. To address this concern, we choose a random sample of 176 cells, one for each country, and compute the correlations. We repeat this procedure 1,000 times and report the resulting cross-country correlations in Column (3). They look similar to those in Column (1) and Column (2).

As a further robustness check, we compute some placebo correlations to be compared with those in Column (1) and Column (3). To do so, we take a different value of $\psi$ when transforming subjective well-being into our measure of utility. In particular, we assume that all countries in the world have the same utility, which is equivalent to identifying fundamental amenities under the assumption that there is free mobility in the world today. The results are reported in Column (4) and Column (5). Note that there is no need to run a similar placebo test on Column (2) since the correlations within countries are independent of our measure of utility. Columns (4) and (1) are similar, but far from identical. This is not surprising since most of the variation in those two columns is across cells within countries, and that variation is unaffected by the particular value of $\psi$. In contrast, when we focus on the variation across countries, using a different value of $\psi$ should change the results. This is indeed what we find: Column (5) yields very different correlations from Column (3). All the signs on proximity to water have switched signs; many of the signs on elevation have also switched signs or give correlations that are statistically insignificant; and the correlation on minimum precipitation changes from positive to negative.

If the fundamental amenities that we have estimated are reasonable, then Columns (1), (2) and (3) should yield similar results, Columns (4) and (1) should not differ too much, and Columns (5) and (3) should be quite different. Our results in Table 6 confirm these priors. Together with the fact that the correlations in Column (1) are in line with those in the literature, this allows us to conclude that our particular methodology of using subjective well-being to identify fundamental amenities performs well.

\section{A.3 Subjective Well-Being}

Figure 13: Cantril Ladder Measure of Subjective Well-being from the Gallup World Poll (Max = 10, Min = 0)

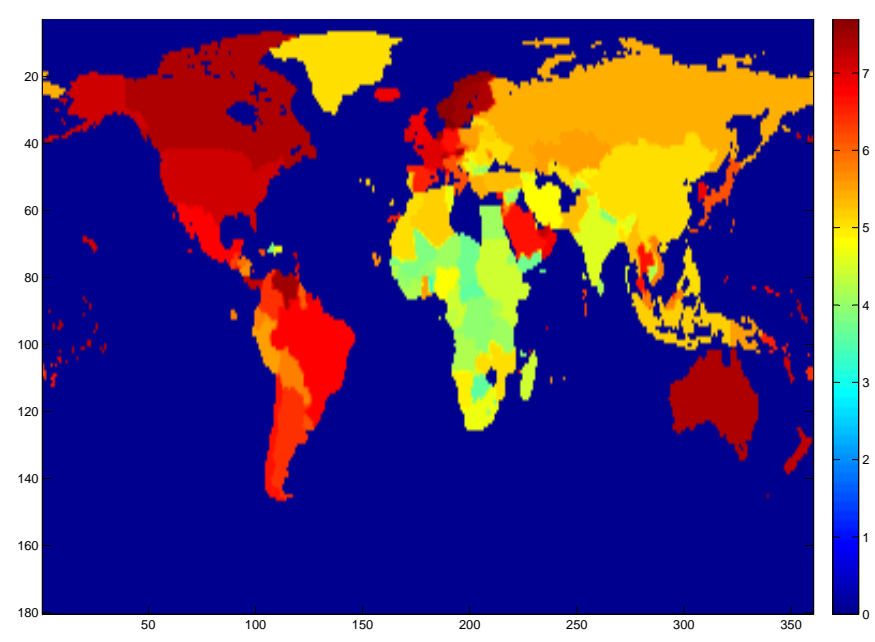




\section{A.4 A Rise in Sea Levels by 1 Meter}

In the next two tables we present results on the impact of a 1-meter rise in the sea level. Tables 8 and 6 correspond to Tables 3 and 4 in the text for this case.

Table 7: The Effect of a Rise in Sea Levels by 1 Meter

\begin{tabular}{lccc}
\hline \hline & Period 1 & Period 100 & Period 500 \\
\hline A. Keeping mobility restrictions unchanged & & & \\
Percentage land flooded & 0.4 & 0.4 & 0.4 \\
Percentage population flooded & 1.6 & 1.3 & 0.8 \\
Percentage land rents lost & 1.9 & 2.1 & 1.2 \\
Percentage technology lost (land weighted) & 0.8 & 0.9 & 0.9 \\
Percentage technology lost (population weighted) & 1.8 & 1.6 & 0.7 \\
Percentage amenities lost (land weighted) & 1.3 & 1.0 & 0.5 \\
Percentage amenities lost (population weighted) & 3.5 & 3.2 & 1.9 \\
\hline B. Free mobility & & & \\
Percentage land flooded & 0.4 & 0.4 & 0.4 \\
Percentage population flooded & 5.5 & 8.5 & 75.5 \\
Percentage land rents lost & 4.2 & 8.5 & 86.3 \\
Percentage technology lost (land weighted) & 0.8 & 1.2 & 3.4 \\
Percentage technology lost (population weighted) & 5.2 & 11.7 & 94.1 \\
Percentage amenities lost (land weighted) & 0.7 & 0.4 & 0.2 \\
Percentage amenities lost (population weighted) & 6.1 & 12.5 & 75.7 \\
\hline C. Partial mobility & & & \\
Percentage land flooded & 0.4 & 0.4 & 0.4 \\
Percentage population flooded & 3.6 & 5.5 & 23.5 \\
Percentage land rents lost (land weighted) & 2.9 & 5.0 & 34.3 \\
Percentage technology lost & 0.8 & 1.1 & 2.1 \\
Percentage technology lost (population weighted) & 3.2 & 7.3 & 58.3 \\
Percentage amenities lost (land weighted) & 1.1 & 0.8 & 0.4 \\
Percentage amenities lost (population weighted) & 5.2 & 9.7 & 49.9 \\
\hline
\end{tabular}

Table 8: Dynamic Effects of Coastal Flooding (1 Meter)

\begin{tabular}{ccccrrc}
\hline \hline Mobility & \multicolumn{2}{c}{ Discounted Present Value of Real Income* } & \multicolumn{3}{c}{ Welfare** $^{* *}$} \\
\cline { 2 - 6 } $0_{0}^{a}$ & No Flooding & Flooding & Ratio (NF/F) & No Flooding & Flooding & Ratio (NF/F) \\
\cline { 2 - 7 } 0.3 & 36.51 & 36.12 & 1.011 & 2.64 & 2.55 & 1.036 \\
0.5 & 37.80 & 37.40 & 1.011 & 4.51 & 4.34 & 1.040 \\
0.9 & 51.59 & 41.20 & 1.010 & 6.11 & 5.87 & 1.041 \\
1.3 & 57.02 & 50.64 & 1.008 & 9.09 & 8.73 & 1.041 \\
$1.8^{b}$ & 61.56 & 60.37 & 1.012 & 10.52 & 10.12 & 1.039 \\
\hline \multicolumn{7}{c}{ a: Observed Restrictions. b: Free Mobility. } \\
*: Normalized by world average GDP without flooding for $t=1$. \\
**: Population-weighted average of cells' utility levels. \\
\hline \hline
\end{tabular}




\section{Appendix B: Proofs, Derivations, and Other Details}

\section{B.1 Derivation of Trade Shares and the Price Index}

We derive here in detail first the probability that a given good produced in an area $r$ is bought in $s$. The area $B(r, \delta)$ (a ball of radius $\delta$ centered at $r$ ) offers different goods in location $s$. The distribution of prices $B(r, \delta$ ) offered in $s$ is given by

$$
G_{t}(p, s, B(r, \delta))=1-e^{-\int_{B(r, \delta)} T_{t}(r)\left[\frac{m c_{t}(r) \varsigma(s, r)}{p}\right]^{-\theta}} d r .
$$

The distribution of the prices of the goods $s$ actually buys is then

$$
G_{t}(p, s)=1-e^{-\int_{S} T_{t}(u)\left[\frac{\left.m c_{t}(u)\right)_{\varsigma}(s, u)}{p}\right]^{-\theta} d u}=1-e^{-\chi_{t}(s) p^{\theta}}
$$

where

$$
\chi_{t}(s)=\int_{S} T_{t}(u)\left[m c_{t}(u) \varsigma(s, u)\right]^{-\theta} d u .
$$

Now we calculate the probability that a given good produced in an area $B(r, \delta)$ is bought in $s$. Start by computing the probability density that the price of the good produced in $B(r, \delta)$ and offered in $s$ is equal to $p$ and that this is the lowest price offered in $s$. This is $\frac{d G_{t}(p, s, B(r, \delta))}{d p} e^{-\int_{S \backslash B(r, \delta)} T_{t}(u)\left[\frac{m c_{t}(u)_{\varsigma}(u, s)}{p}\right]^{-\theta} d u}$. Re-write $d G_{t}(p, s, B(r, \delta))=$ $e^{-\int_{B(r, \delta)} T_{t}(r)\left[\frac{m c_{t}(r) \varsigma(s, r)}{p}\right]^{-\theta} d r} \int_{B(r, \delta)} T_{t}(r)\left[m c_{t}(r) \varsigma(r, s)\right]^{-\theta} d r \theta p^{\theta-1} d p$. Replace this into the previous expression and integrate over all possible prices, $\int_{0}^{\infty} e^{-\int_{S} T_{t}(u)\left[\frac{m c_{t}(u) \varsigma(s, u)}{p}\right]^{-\theta} d u} \int_{B(r, \delta)} T_{t}(r)\left[m c_{t}(r) \varsigma(r, s)\right]^{-\theta} d r \theta p^{\theta-1} d p$. Solving this integral yields $\left[-e^{-\chi_{t}(s) p^{\theta}} \frac{\int_{B(r, \delta)} T_{t}(r)\left[m c_{t}(r) \varsigma(r, s)\right]^{-\theta} d r}{\chi_{t}(s)}\right]_{0}^{\infty}$, which gives

$$
\pi_{t}(s, B(r, \delta))=\frac{\int_{B(r, \delta)} T_{t}(r)\left[m c_{t}(r) \varsigma(r, s)\right]^{-\theta} d r}{\int_{S} T_{t}(u)\left[m c_{t}(u) \varsigma(u, s)\right]^{-\theta} d u}=\frac{\int_{B(r, \delta)} T_{t}(r)\left[m c_{t}(r) \varsigma(r, s)\right]^{-\theta} d r}{\chi_{t}(s)} \text { all } r, s \in S .
$$

Since in any small interval with positive measure there will be many firms producing many goods, the above expressions can be interpreted as the fraction of goods location $s$ buys from $B(r, \delta)$. In the limit, as $\delta \rightarrow 0$, this expression can be rewritten as

$$
\pi_{t}(s, r)=\frac{T_{t}(r)\left[m c_{t}(r) \varsigma(r, s)\right]^{-\theta}}{\int_{S} T_{t}(u)\left[m c_{t}(u) \varsigma(u, s)\right]^{-\theta} d u}=\frac{T_{t}(r)\left[m c_{t}(r) \varsigma(r, s)\right]^{-\theta}}{\chi_{t}(s)} \text { all } r, s \in S,
$$

which is the expression in the text.

To finish this subsection, we derive the price index of a location $s$. We know that $P_{t}(s)^{\frac{-\rho}{1-\rho}}=\int_{0}^{1} p_{t}^{\omega}(s)^{\frac{-\rho}{1-\rho}} d \omega$. This can be interpreted as the average price computed over the different goods that are being sold in $s$. This is the same as the expected value of the price of any good sold in $s$, so that $P_{t}(s)^{\frac{-\rho}{1-\rho}}=\int_{0}^{\infty} p^{\frac{-\rho}{1-\rho}} \frac{d G_{t}(s)}{d p} d p$. Since $\frac{d G_{t}(s)}{d p}=\chi_{t}(s) \theta p^{\theta-1} e^{-\chi_{t}(s) p^{\theta}}$, we can write the previous expression as $P_{t}(s)^{\frac{-\rho}{1-\rho}}=\int_{0}^{\infty} p^{\frac{-\rho}{1-\rho}} \chi_{t}(s) \theta p^{\theta-1} e^{-\chi_{t}(s) p^{\theta}} d p$. By defining $x=\chi_{t}(s) p^{\theta}$, we can write $d x=\theta p^{\theta-1} \chi_{t}(s)$. Substituting yields $\int_{0}^{\infty}\left[\frac{x}{\chi_{t}(s)}\right]^{\frac{-\rho}{(1-\rho) \theta}} e^{-x} d x$ which is equal to $\chi_{t}(s)^{\frac{\rho}{(1-\rho) \theta}} \int_{0}^{\infty} x^{\frac{-\rho}{(1-\rho) \theta}} e^{-x} d x$. The $\Gamma$ function is defined as $\Gamma(\alpha)=\int_{0}^{\infty} y^{\alpha-1} e^{-y} d y$, so that we can rewrite the 
above expression as $\chi_{t}(s)^{\frac{\rho}{(1-\rho) \theta}} \Gamma\left(\frac{-\rho}{(1-\rho) \theta}+1\right)$. Therefore $P_{t}(s)^{\frac{-\rho}{1-\rho}}=\chi_{t}(s)^{\frac{\rho}{(1-\rho) \theta}} \Gamma\left(\frac{-\rho}{(1-\rho) \theta}+1\right)$, so that

$$
P_{t}(s)=\chi_{t}(s)^{\frac{-1}{\theta}}\left[\Gamma\left(\frac{-\rho}{(1-\rho) \theta}+1\right)\right]^{-\frac{1-\rho}{\rho}}
$$

which is the expression in the text.

Proof of Lemma 2. Substituting (16) and (45) into (18), we obtain

$$
\bar{u}_{t}^{W} \bar{u}(c)=\bar{a}(r) \bar{L}_{t}(r)^{-\lambda} \frac{\xi}{\mu \xi+\gamma_{1}} \frac{w_{t}(r)}{\left[\sum_{d=1}^{C} \int_{S_{d}} T_{t}(s)\left[m c_{t}(s) \varsigma(r, s)\right]^{-\theta} d s\right]^{\frac{-1}{\theta}} \bar{p}}
$$

for any location $r \in S_{c}$, where $\bar{p}=\left[\Gamma\left(\frac{-\rho}{(1-\rho) \theta}+1\right)\right]^{-\frac{1-\rho}{\rho}}$. We can rewrite this as

$$
\bar{a}(r)^{-\theta} w_{t}(r)^{-\theta} \bar{L}_{t}(r)^{\lambda \theta}=\left[\frac{\mu \xi+\gamma_{1}}{\xi}\right]^{-\theta}\left(\bar{u}_{t}^{W} \bar{u}(c) \bar{p}\right)^{-\theta} \sum_{d=1}^{C} \int_{S_{d}} T_{t}(s)\left(c_{t}(s) \varsigma(r, s)\right)^{-\theta} d s,
$$

from which

$$
\left[\frac{\bar{a}(r)}{\bar{u}(c)}\right]^{-\theta} w_{t}(r)^{-\theta} \bar{L}_{t}(r)^{\lambda \theta}\left(\bar{u}_{t}^{W}\right)^{\theta}=\kappa_{1} \sum_{d=1}^{C} \int_{S_{d}} \tau_{t}(s) \varsigma(r, s)^{-\theta} w_{t}(s)^{-\theta} \bar{L}_{t}(s)^{\alpha-\left(1-\mu-\frac{\gamma_{1}}{\xi}\right) \theta} d s
$$

where $\kappa_{1}=\left[\frac{\mu \xi+\gamma_{1}}{\xi}\right]^{-\left[\mu+\frac{\gamma_{1}}{\xi}\right] \theta} \mu^{\mu \theta}\left[\frac{\xi \nu}{\gamma_{1}}\right]^{-\frac{\gamma_{1} \theta}{\xi}} \bar{p}^{-\theta}$. This yields the first set of equations that $L_{t}(\cdot)$ and $w_{t}(\cdot)$ have to solve.

Inserting (15) and (16) into the balanced trade condition, we get

$$
w_{t}(r) H(r) \bar{L}_{t}(r)=\bar{p}^{-\theta} \sum_{d=1}^{C} \int_{S_{d}} T_{t}(r)\left[m c_{t}(r) \varsigma(r, s)\right]^{-\theta} P_{t}(s)^{\theta} w_{t}(s) H(s) \bar{L}_{t}(s) d s
$$

Substituting (18) and $T_{t}(r)=\tau_{t}(r) \bar{L}_{t}(r)^{\alpha}$ into the previous equation yields

$$
\tau_{t}(r)^{-1} H(r) w_{t}(r)^{1+\theta} \bar{L}_{t}(r)^{1-\alpha+\left(1-\mu-\frac{\gamma_{1}}{\xi}\right) \theta}\left(\bar{u}_{t}^{W}\right)^{\theta}=\kappa_{1} \sum_{d=1}^{C} \int_{S_{d}}\left[\frac{\bar{a}(s)}{\bar{u}(d)}\right]^{\theta} H(s) \varsigma(s, r)^{-\theta} w_{t}(s)^{1+\theta} \bar{L}_{t}(s)^{1-\lambda \theta} d s .
$$

This gives the second set of equations that $L_{t}(\cdot)$ and $w_{t}(\cdot)$ have to solve.

We can use (46) and (47) to analyze the equilibrium allocation further under symmetric trade costs. So assume trade costs are symmetric so that $\varsigma(r, s)=\varsigma(s, r)$. Now guess that $w_{t}(r)=\bar{w}_{t}\left[\frac{\bar{a}(r)}{\bar{u}(c)}\right]^{\iota_{1}} \tau_{t}(r)^{\iota_{2}} H(r)^{\iota_{3}} \bar{L}_{t}(r)^{\iota_{4}}$ for any location $r \in S_{c}$. Substituting into (46) and (47) and using the symmetry of trade costs, one can verify the guess if

$$
\iota_{1}=-\frac{\theta}{1+2 \theta}, \quad \iota_{2}=\frac{1}{1+2 \theta}, \quad \iota_{3}=-\frac{1}{1+2 \theta}, \quad \text { and } \quad \iota_{4}=\frac{\alpha-1+\left[\lambda+\frac{\gamma_{1}}{\xi}-(1-\mu)\right] \theta}{1+2 \theta} .
$$

Hence, wages at location $r$ are a function of amenities, productivity, land, and population density. Namely, as 
stated in Lemma (2),

$$
w_{t}(r)=\bar{w}\left[\frac{\bar{a}(r)}{\bar{u}(c)}\right]^{-\frac{\theta}{1+2 \theta}} \tau_{t}(r)^{\frac{1}{1+2 \theta}} H(r)^{-\frac{1}{1+2 \theta}} \bar{L}_{t}(r)^{\frac{\alpha-1+\left[\lambda+\frac{\gamma_{1}}{\xi}-(1-\mu)\right] \theta}{1+2 \theta}} .
$$

Substituting in 46 then yields the second equation in Lemma 22,

$$
\begin{aligned}
& {\left[\frac{\bar{a}(r)}{\bar{u}(c)}\right]^{-\frac{\theta(1+\theta)}{1+2 \theta}} \tau_{t}(r)^{-\frac{\theta}{1+2 \theta}} H(r)^{\frac{\theta}{1+2 \theta}} \bar{L}_{t}(r)^{\lambda \theta-\frac{\theta}{1+2 \theta}}\left[\alpha-1+\left[\lambda+\frac{\gamma_{1}}{\xi}-(1-\mu)\right] \theta\right] } \\
= & {\left[\bar{u}_{t}^{W}\right]^{-\theta} \kappa_{1} \sum_{d=1}^{C} \int_{S_{d}}\left[\frac{\bar{a}(s)}{\bar{u}(d)}\right]^{\frac{\theta^{2}}{1+2 \theta}} \tau_{t}(s)^{\frac{1+\theta}{1+2 \theta}} H(s)^{\frac{\theta}{1+2 \theta}} \varsigma(r, s)^{-\theta} \bar{L}_{t}(s)^{1-\lambda \theta+\frac{1+\theta}{1+2 \theta}\left[\alpha-1+\left[\lambda+\frac{\gamma_{1}}{\xi}-(1-\mu)\right] \theta\right]} d s, }
\end{aligned}
$$

which pins down the function $\bar{L}_{t}(\cdot)$.

Proof of Lemma 4. Given the evolution of technology in (19), the growth rate of $\tau_{t}(r)$ is given by

$$
\frac{\tau_{t+1}(r)}{\tau_{t}(r)}=\phi_{t}(r)^{\theta \gamma_{1}}\left[\int_{S} \eta \frac{\tau_{t}(s)}{\tau_{t}(r)} d s\right]^{1-\gamma_{2}} \text {. }
$$

Divide both sides of the equation by the corresponding equation for location $s$, and rearrange to get

$$
\frac{\tau_{t}(s)}{\tau_{t}(r)}=\left[\frac{\phi(s)}{\phi(r)}\right]^{\frac{\theta \gamma_{1}}{1-\gamma_{2}}}=\left[\frac{\bar{L}(s)}{\bar{L}(r)}\right]^{\frac{\theta \gamma_{1}}{\left(1-\gamma_{2}\right) \xi}}
$$

where the second equality follows from $(8)$ and where we drop the time subscript to indicate that we refer to a variable that remains constant in the BGP. Use this relationship to obtain that

$$
\bar{L}(s)=\left[\frac{\tau_{t}(s)}{\tau_{t}(r)}\right]^{\frac{\left(1-\gamma_{2}\right) \xi}{\theta \gamma_{1}}} \bar{L}(r)
$$

and so, after integrating over $s$ and using the labor market clearing condition, that

$$
\int_{S} \bar{L}(s) d s=\bar{L}=\bar{L}(r) \tau_{t}(r)^{-\frac{\left(1-\gamma_{2}\right) \xi}{\theta \gamma_{1}}} \int_{S} \tau_{t}(s)^{\frac{\left(1-\gamma_{2}\right) \xi}{\theta \gamma_{1}}} d s
$$

or

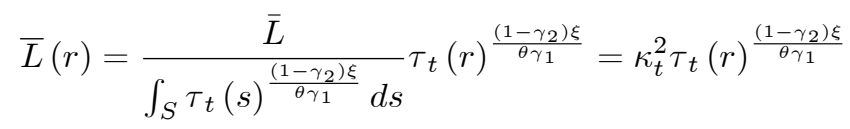

where $\kappa_{t}^{2}$ depends on time but not on location.

In equilibrium Lemma 3 (plus the power guess for wages) implies that the BGP labor density $\bar{L}(\cdot)$ has to satisfy

$$
\begin{aligned}
& {\left[\frac{\bar{a}(r)}{\bar{u}(c)}\right]^{-\frac{\theta(1+\theta)}{1+2 \theta}} \tau_{t}(r)^{-\frac{\theta}{1+2 \theta}} H(r)^{\frac{\theta}{1+2 \theta}} \bar{L}(r)^{\lambda \theta-\frac{\theta}{1+2 \theta}\left[\alpha-1+\left[\lambda+\frac{\gamma_{1}}{\xi}-[1-\mu]\right] \theta\right]} } \\
= & {\left[\bar{u}_{t}^{W}\right]^{-\theta} \kappa_{1} \sum_{d=1}^{C} \int_{S_{d}}\left[\frac{\bar{a}(s)}{\bar{u}(d)}\right]^{\frac{\theta^{2}}{1+2 \theta}} \tau_{t}(s)^{\frac{1+\theta}{1+2 \theta}} H(s)^{\frac{\theta}{1+2 \theta}} \varsigma(r, s)^{-\theta} \bar{L}(s)^{1-\lambda \theta+\frac{1+\theta}{1+2 \theta}\left[\alpha-1+\left[\lambda+\frac{\gamma_{1}}{\xi}-[1-\mu]\right] \theta\right]} d s }
\end{aligned}
$$


and so

$$
\begin{aligned}
& {\left[\frac{\bar{a}(r)}{\bar{u}(c)}\right]^{-\frac{\theta(1+\theta)}{1+2 \theta}} H(r)^{\frac{\theta}{1+2 \theta}} \tau_{t}(r)^{\frac{\left[1-\gamma_{2}\right] \xi}{\theta \gamma_{1}}\left[\lambda \theta-\frac{\theta}{1+2 \theta}\left[\alpha-1+\left[\lambda+\frac{\gamma_{1}}{\xi}-[1-\mu]\right] \theta\right]\right]-\frac{\theta}{1+2 \theta}}} \\
& =\left[\bar{u}_{t}^{W W}\right]^{-\theta} \kappa_{t}^{3} \sum_{d=1}^{C} \int_{S_{d}}\left[\frac{\bar{a}(s)}{\bar{u}(d)}\right]^{\frac{\theta^{2}}{1+2 \theta}} H(s)^{\frac{\theta}{1+2 \theta}} \varsigma(r, s)^{-\theta} \tau_{t}(s)^{\frac{\left[1-\gamma_{2}\right] \xi}{\theta \gamma_{1}}\left[1-\lambda \theta+\frac{1+\theta}{1+2 \theta}\left[\alpha-1+\left[\lambda+\frac{\gamma_{1}}{\xi}-[1-\mu]\right] \theta\right]\right]+\frac{1+\theta}{1+2 \theta}} d s \text {, }
\end{aligned}
$$

where $\kappa_{t}^{3}=\kappa_{1}\left[\kappa_{t}^{2}\right]^{\alpha+\left[\frac{\gamma_{1}}{\xi}-\lambda-(1-\mu)\right] \theta}$.

The solution to this integral equation is the spatial distribution of productivity $\tau_{t}(r)$ on the balanced growth path. According to Theorem 2.19 in Zabreyko, et al. (1975) a unique positive solution exists if

$$
\frac{\alpha}{\theta}+\frac{\gamma_{1}}{\xi}+\frac{\gamma_{1}}{\left[1-\gamma_{2}\right] \xi} \leq \lambda+1-\mu
$$

Proof of Lemma 5. Lemma 4 guarantees that if the economy is in its BGP in periods $t$ and $t+1$, then

$$
\begin{aligned}
\frac{\bar{u}_{t+1}^{W}}{\bar{u}_{t}^{W}} & =\left[\frac{\kappa_{t+1}^{2}}{\kappa_{t}^{2}}\right]^{\frac{\alpha}{\theta}+\frac{\gamma_{1}}{\xi}-\lambda-[1-\mu]}\left[\frac{\tau_{t+1}(r)}{\tau_{t}(r)}\right]^{\frac{1}{\theta}+\frac{\left[1-\gamma_{2}\right] \xi}{\theta \gamma_{1}}\left[\frac{\alpha}{\theta}+\frac{\gamma_{1}}{\xi}-\lambda-(1-\mu)\right]} \\
& =\left[\frac{\tau_{t+1}(r)}{\tau_{t}(r)}\right]^{\frac{1}{\theta}}
\end{aligned}
$$

for all $r$ and so since

$$
\begin{aligned}
\frac{\tau_{t+1}(r)}{\tau_{t}(r)} & =\phi(r)^{\theta \gamma_{1}}\left[\int_{S} \eta \frac{\tau_{t}(s)}{\tau_{t}(r)} d s\right]^{1-\gamma_{2}}=\phi(r)^{\theta \gamma_{1}}\left[\int_{S} \eta\left[\frac{\phi(s)}{\phi(r)}\right]^{\frac{\theta \gamma_{1}}{1-\gamma_{2}}} d s\right]^{1-\gamma_{2}} \\
& =\eta^{1-\gamma_{2}}\left[\frac{\gamma_{1} / \nu}{\gamma_{1}+\mu \xi}\right]^{\frac{\theta \gamma_{1}}{\xi}}\left[\int_{S} \bar{L}(s)^{\frac{\theta \gamma_{1}}{\left[1-\gamma_{2}\right] \xi}} d s\right]^{1-\gamma_{2}}
\end{aligned}
$$

we have that

$$
\frac{\bar{u}_{t+1}^{W}}{\bar{u}_{t}^{W}}=\eta^{\frac{1-\gamma_{2}}{\theta}}\left[\frac{\gamma_{1} / \nu}{\gamma_{1}+\mu \xi}\right]^{\frac{\gamma_{1}}{\xi}}\left(\int_{S} \bar{L}(s)^{\frac{\theta \gamma_{1}}{\left[1-\gamma_{2}\right] \xi}} d s\right)^{\frac{1-\gamma_{2}}{\theta}}
$$

\section{B.2 Procedure to Find a Solution to Equation (29)}

We use the following procedure to solve equation 229 . We approximate 229 by

$$
w_{0}(r)^{-\theta} \bar{L}_{0}(r)^{\lambda \theta}\left[\frac{\bar{a}(r)}{\bar{u}(c)}\right]^{-\theta}=\left[\bar{u}_{0}^{W}\right]^{-\theta} \kappa_{1} \bar{w}^{-[1+2 \theta]} \sum_{d=1}^{C} \int_{S_{d}} w_{0}(s)^{1+\theta} \bar{L}_{0}(s)^{1-\lambda \theta} H(s) \varsigma(r, s)^{-\theta}\left[\frac{\bar{a}(s)}{\bar{u}(d)}\right]^{\theta-\epsilon^{0}} d s
$$

where $\epsilon^{0}>0$ is a constant. For any positive $\epsilon^{0}$, Theorem 2.19 in Zabreyko et al. (1975) guarantees that 49 can be solved by the following simple iterative procedure. Guess some initial distribution $\left[\frac{\bar{a}(r)}{\bar{u}(c)}\right]_{\epsilon^{0}}^{0}$, plug it into the 
right-hand side of equation 49 , calculate the left-hand side, and solve for $\bar{a}(r) / \bar{u}(c)$; call this $\left[\frac{\bar{a}(r)}{\bar{u}(c)}\right]_{\epsilon^{0}}^{1}$. Compute the distance between $\left[\frac{\bar{a}(r)}{\bar{u}(c)}\right]_{\epsilon^{0}}^{1}$ and $\left[\frac{\bar{a}(r)}{\bar{u}(c)}\right]_{\epsilon^{0}}^{0}$, defined as

$$
d i s t^{1}=\int_{S}\left[\left[\frac{\bar{a}(r)}{\bar{u}(c)}\right]_{\epsilon^{0}}^{1}-\left[\frac{\bar{a}(r)}{\bar{u}(c)}\right]_{\epsilon^{0}}^{0}\right]^{2} d r .
$$

If $d i s t^{1}<\varepsilon$ where $\varepsilon$ is an exogenously given tolerance level, stop. Otherwise, plug $\left[\frac{\bar{a}(r)}{\bar{u}(c)}\right]_{\epsilon^{0}}^{1}$ into the right-hand side of 49 , express $\bar{a}(r) / \bar{u}(c)$ from the left-hand side, that is, obtain $[\overline{\bar{a}(r)}]_{\bar{u}(c)}^{2}$, and compute $\operatorname{cist}^{2}$, defined analogously to dist ${ }^{1}$. Continue the procedure until dist ${ }^{i}<\varepsilon$ for some $i$.

Having the solution to $\sqrt[49]{ },\left[\frac{\bar{a}(r)}{\bar{u}(c)}\right]_{\epsilon_{0}}$, one needs to check whether it is sufficiently close to the solution of 29 . Given that the system of (21) and (20) is equivalent to the system of $(29)$ and (28), we can check this in the following way. First, we solve for $\tau_{0}(r)_{\epsilon^{0}}$ using $\left[\frac{\bar{a}(r)}{\bar{u}(c)}\right]_{\epsilon^{0}}$ and equation 28 . Next, we plug $\left[\frac{\bar{a}(r)}{\bar{u}(c)}\right]_{\epsilon^{0}}$ and $\tau_{0}(r)_{\epsilon^{0}}$ into 21), and solve for population levels $\bar{L}_{0}(r)_{\epsilon^{0}}{ }^{36}$ Finally, we check whether $\bar{L}_{0}(r)_{\epsilon^{0}}$ are sufficiently close to $\bar{L}_{0}(r)$, the population levels seen in the data ${ }^{37}$ If they are not close enough, we take $\epsilon^{1}=\epsilon^{0} / 2$, and redo the whole procedure with $\epsilon^{1}$ instead of $\epsilon^{0}$. Now, if $\bar{L}_{0}(r)_{\epsilon^{1}}$ are sufficiently close to $\bar{L}_{0}(r)$, we stop. Otherwise, we proceed with $\epsilon^{2}=\epsilon^{1} / 2$; and so on. This procedure results in a pair of distributions $\left[\frac{\bar{a}(r)}{\bar{u}(c)}\right]_{\epsilon^{i}}$ and $\tau_{0}(r)_{\epsilon^{i}}$ which are sufficiently close to the solution of 29$]$ and 28$]$.

\section{Appendix C: Data Appendix}

The data description and sources follow approximately the order in which they appear in the paper. For the numerical exercise, all data are essentially for the time period 2000-2010, whereas for the calibration of the parameters we sometimes use data from a longer time period.

Population and amenities of U.S. Metropolitan Statistical Areas. Data on population of U.S. MSAs come from the Bureau of Economic Analysis, Regional Economic Accounts, and are for the years 2005 and 2008. Corresponding data on amenities are estimated by the structural model in Desmet and Rossi-Hansberg (2013).

Population, GDP and wages at $1^{\circ}$ by $1^{\circ}$ resolution. Data on population and GDP for $1^{\circ}$ by $1^{\circ}$ cells for the entire world come from the G-Econ 4.0 research project at Yale University. For the calibration of the technology parameters, we use data of 1990, 1995, 2000 and 2005 and take GDP measured in PPP terms. For the benchmark model, we use data of 2000. Wages are taken to be GDP in PPP terms divided by population.

Railroads, major roads, other roads and waterways at $1^{\circ}$ by $1^{\circ}$ resolution. Data on railroads, major roads and other roads come from http://www.naturalearthdata.com/, a public domain map data set built through the collaborative effort of many volunteers and supported by the North American Cartographic Information

\footnotetext{
${ }^{36}$ This is done using the procedure explained in Section 4.7 .

${ }^{37}$ The criterion used here is

$$
\widetilde{d i s t^{0}}=\int_{S}\left[\bar{L}_{0}(r)_{\epsilon^{0}}-\bar{L}_{0}(r)\right]^{2} d r<\widetilde{\varepsilon}
$$
}

where $\widetilde{\varepsilon}$ is a positive tolerance level. 
Society. For each $1^{\circ}$ by $1^{\circ}$ cell, we define railroads as the share of $0.01^{\circ}$ by $0.01^{\circ}$ cells through which a railroad passes. Major roads, other roads and waterways are defined analogously. Major roads refer to a major highway, a beltway or a bypass; other roads refer to any other type of road; and waterways refer to either a river or an ocean.

Land at $1^{\circ}$ by $1^{\circ}$ and $30^{\prime \prime}$ by $30^{\prime \prime}$ resolution. Data on land come from the Global Land One-km Base Elevation (GLOBE) digital elevation model (DEM), a raster elevation data set from the National Oceanic and Atmospheric Administration (NOAA) covering all $30^{\prime \prime}$ by $30^{\prime \prime}$ cells that are located on land. Using this information, we compute for each $1^{\circ}$ by $1^{\circ}$ cell on the globe the share of the $30^{\prime \prime}$ by $30^{\prime \prime}$ cells that are on land.

Subjective well-being at country level. Subjective well-being is measured on a Cantril ladder from 0 to 10, where 0 represents the worst possible life and 10 the best possible life the individual can contemplate for himself. The main data source is the Gallup World Poll, and we take the mean for the period 2007-2011 as reported in the Human Development Report 2013. This gives us data on 151 countries. To increase the coverage of countries, we proceed in three ways. First, we use the database by Veenhoven (2013) who reports data for a similar time period on the same evaluative measure of subjective well-being from Gallup, the Pew Research Center and Latinobarómetro. This gives an additional 7 countries. Second, Abdallah, Thompson and Marks (2008) propose a model to estimate subjective measures of well-being for countries that are typically not surveyed. To make the data comparable, we normalize the Abdallah et al. measure so that the countries that are common with those surveyed by Gallup have the same means and standard deviations. This increases the coverage to 184 countries. Third, for the rest of the world - mostly small islands and territories - we assign subjective well-being measures in an ad-hoc manner. For example, for Andorra we take the average of France and Spain. For another example, in the case of Nauru, a small island in the Pacific, we take the average of the Solomon Islands and Vanuatu.

Desired and actual migration at country level. Data on desired migration come from the Gallup World Poll, based on surveys starting in 2007. In particular, we have information on (i) how many people would like to permanently migrate; (ii) which country is the favorite destination; (iii) and of those who want to migrate, the share that chooses the favorite destination. Bilateral data on actual migration come from Artuc et al. (2012) and are for 2000. To calculate shares of actual migrants, we use population data from the World Development Indicators, also for 2000.

Rise in sea levels at $\mathbf{3 0}^{\prime \prime}$ by $\mathbf{3 0}^{\prime \prime}$ resolution. The raster data on inundation come from the Center for Remote Censoring of Ice Sheets (Cresis). Inundation zones from a 6-meter rise in sea level were calculated by Cresis from the same Global Land One-km Base Elevation digital elevation model of the NOAA mentioned before. The resolution is $30^{\prime \prime}$ by $30^{\prime \prime}$.

Population at $\mathbf{3 0}^{\prime \prime}$ by $\mathbf{3 0}^{\prime \prime}$ resolution. Population data at the same resolution of 30 arcsecond $\mathrm{x} 30$ arcsecond come from LandScan 2005 at the Oak Ridge National Laboratory.

Population (and share of population) flooded at $1^{\circ}$ by $1^{\circ}$ resolution. Using population data and flooding data at a resolution of $30^{\prime \prime}$ by $30^{\prime \prime}$, we compute how many people are flooded at a resolution of $30^{\prime \prime}$ by $30^{\prime \prime}$, and then aggregate up to a resolution of $1^{\circ}$ by $1^{\circ}$. It is then easy to compute the share of population flooded. 
Share of land flooded at $1^{\circ}$ by $1^{\circ}$ resolution. The share of land flooded at $1^{\circ}$ by $1^{\circ}$ resolution is defined as the number of cells at $30^{\prime \prime}$ by $30^{\prime \prime}$ resolution (contained in each $1^{\circ}$ by $1^{\circ}$ cell) that get flooded divided by the number of cells at $30^{\prime \prime}$ by $30^{\prime \prime}$ resolution (contained in each $1^{\circ}$ by $1^{\circ}$ cell) that are on land.

U.S. zip code and CBSA data. Data on area, population, mean earnings per worker and income per capita come from the U.S. census. Data referred to as 2000 are from the 2000 Census and data referred to as 2007-2011 come from the 2007-2011 American Community Survey 5-year estimates. The geographic unit of observation is the ZIP Code Tabulation Areas (ZCTAs).

Data on payroll and number of employees for U.S. zip codes in 2010 come from the ZIP Code Business Patterns from the U.S. Census. Note that zip codes and ZCTAs are not exactly the same. In particular, zip codes do not have areas. ZCTAs should be understood as areal representations of United States Postal Service zip codes. When calculating employee density for zip codes, we therefore use the corresponding ZCTAs. To match ZCTAs to Core Based Statistical Areas (CBSA) we rely on tabulation files from the U.S. Census.

\section{References}

[1] Abdallah, S., Thompson, S. and Marks, N., 2008. "Estimating Worldwide Life Satisfaction," Ecological Economics, 65, 35-47.

[2] Albouy, D., Graf, W., Kellogg, R., and Wolff, H., 2014. "Climate Amenities, Climate Change, and American Quality of Life," unpublished manuscript.

[3] Allen, T. and Arkolakis, C., 2014. "Trade and the Topography of the Spatial Economy," Quarterly Journal of Economics, forthcoming.

[4] Anthoff, D., Nicholls, R.J., Morgenroth, E.L.W., and Tol, R.S.J., 2010. "The Economic Impact of Substantial Sea-Level Rise," Mitigation and Adaptation Strategies for Global Change, 15, 321-335.

[5] Arnell, N.W., Livermore, M.J.L., Kovats, S., Levy, P.E., Nicholls, R., Parry, M.L., Gaffin, S.R., 2004. "Climate and Socio-Economic Scenarios for Global-Scale Climate Change Impacts Assessments: Characterising the SRES Storylines," Global Environmental Change, 14, 3-20.

[6] Artuc, E., Docquier, F., Ozden, C., and Parsons, C., 2013. "A Global Assessment of Human Capital Mobility: The Role of Non-OECD Destinations." Data at http://perso.uclouvain.be/frederic.docquier/ oxlight.htm (accessed September 18, 2013).

[7] Behrens, K., Mion. G., Murata, Y., and Südekum, J., 2013. "Spatial Frictions," unpublished manuscript.

[8] Bernard, A.B., Eaton, J., Jensen, B.J., and Kortum, S., 2003. "Plants and Productivity in International Trade," American Economic Review, 93, 1268-1290.

[9] Caliendo, L., Parro, F., Sarte, P. and Rossi-Hansberg, E., 2014. "The Impact of Regional and Sectoral Productivity Changes in the U.S. Economy," unpublished manuscript. 
[10] Church, J., Clark, P. Cazenave, A., Gregory, J., Jevrejeva, S., Levermann, A., Merrifield, M., Milne, G., Nerem, R., Nunn, P., Payne, A., Pfeffer, W., Stammer, D., and Unnikrishnan, A., 2013. "Sea Level Change," in: Climate Change 2013: The Physical Science Basis. Contribution of Working Group I to the Fifth Assessment Report of the Intergovernmental Panel on Climate Change, Stocker, T., D. Qin, G.-K. Plattner, M. Tignor, S. Allen, J. Boschung, A. Nauels, Y. Xia, Bex, V. and Midgley, P. Eds., Cambridge UP.

[11] Ciccone, A., and Hall, R.E., 1996. "Productivity and the Density of Economic Activity," American Economic Review, 86, 54-70.

[12] Dasgupta, S., Laplante, B., Meisner, C., Wheeler, D. \& Jianping Y., 2007. "The Impact of Sea Level Rise on Developing Countries: A Comparative Analysis," Policy Research Working Paper Series 4136, World Bank.

[13] Davis, M.A., and Ortalo-Magné, 2007. "Wages, Rents, Quality of Life," unpublished manuscript.

[14] Deaton, A., 2008. "Income, Health, and Well-Being around the World: Evidence from the Gallup World Poll," Journal of Economic Perspectives, 22, 53-72.

[15] Deaton, A. and Stone, A., 2013. "Two Happiness Puzzles," American Economic Review, 103:3, 591-97.

[16] Desmet, K. and Rappaport, J., 2014. "The Settlement of the United States, 1800 to 2000: The Long Transition Towards Gibrat's Law," unpublished manuscript.

[17] Desmet, K. and Rossi-Hansberg, E., 2014a. "Spatial Development," American Economic Review, 104:4, 12111243.

[18] Desmet, K. and Rossi-Hansberg, E., 2014b. "On the Spatial Economic Impact of Global Warming," unpublished manuscript.

[19] Desmet, K. and Rossi-Hansberg, E., 2013. "Urban Accounting and Welfare," American Economic Review, 103:6, 2296-2327.

[20] Docquier, F., Machado, J., and Sekkat, K., 2012. "Efficiency Gains from Liberalizing Labor Mobility," IRES Discussion Paper 2012-23.

[21] Eaton, J. and Kortum, S., 2002. "Technology, Geography, and Trade," Econometrica, 70, 1741-1779.

[22] Fajgelbaum, P. and Redding, S. 2014. "External Integration, Structural Transformation and Economic Development: Evidence from Argentina 1870-1914," unpublished manuscript.

[23] Glaeser, E.L., Gottlieb, J.D., and Ziv, O., 2014. "Unhappy Cities," Journal of Labor Economics, forthcoming.

[24] Greenwood, J., Hercowitz, Z., and Krusell, P., 1997. "Long-Run Implications of Investment-Specific Technological Change," American Economic Review, 87, 342-362.

[25] Grogger, J. and Hanson, G.H., 2011. "Income Maximization and the Selection and Sorting of International Migrants," Journal of Development Economics, 95, 42-57.

[26] Hinkel, J. and Klein, R.T., 2009. "Integrating Knowledge to Assess Coastal Vulnerability to Sea-Level Rise: The Development of the DIVA Tool," Global Environmental Change, 19, 384-395. 
[27] Kahneman, D. and Deaton, A., 2010. "High Income Improves Evaluation of Life but Not Emotional Wellbeing," Proceedings of the National Academies of Science, 107 (38): 16489-93.

[28] Kennan, J., 2013. "Open Borders," Review of Economic Dynamics, 16, L1-L13.

[29] Klein, P. and Ventura, G., 2007. "TFP Differences and the Aggregate Effects of Labor Mobility in the Long Run," B.E. Journal of Macroeconomics, 7, 1-36.

[30] Kline, P., and Moretti, E., 2014. "Local Economic Development, Agglomeration Economies and the Big Push: 100 Years of Evidence from the Tennessee Valley Authority," Quarterly Journal of Economics, 129, 275-331.

[31] Moser, S.C., Davidson, M.A., Kirshen, P., Mulvaney, P., Murley, J.F., Neumann, J.E., Petes, L., and Reed, D., 2014. "Ch. 25: Coastal Zone Development and Ecosystems," Climate Change Impacts in the United States: The Third National Climate Assessment, J. M. Melillo, Terese (T.C.) Richmond, and G. W. Yohe, Eds., U.S. Global Change Research Program, 579-618.

[32] Nicholls, R.J., 2004. "Coastal Flooding and Wetland Loss in the 21st Century: Changes under the SRES Climate and Socio-Economic Scenarios," Global Environmental Change, 14, 69-86.

[33] Nicholls, R.J., Hanson, S., Herweijer, C., Patmore, N., Hallegatte, S., Corfee-Morlot, J., Chateau, J., and Muir-Wood, R., 2008. "Ranking Port Cities with High Exposure and Vulnerability to Climate Extremes: Exposure Estimates," OECD Environment Working Papers, No. 1, OECD Publishing.

[34] Roback, J., 1982. "Wages, Rents, and the Quality of Life," Journal of Political Economy, 90:6, 1257-1278.

[35] Rosen, Sherwin. 1979. "Wage-Based Indexes of Urban Quality of Life," in Current Issues in Urban Economics, edited by Peter M. Mieszowski and Mahlon R. Straszheim, 74-104. Johns Hopkins UP.

[36] Simonovska, I. and Waugh, M.E., 2014. "Trade Models, Trade Elasticities, and the Gains from Trade," unpublished manuscript.

[37] Stevenson, B. and Wolfers, J., 2013. "Subjective Well-Being and Income: Is There Any Evidence of Satiation?," American Economic Review, 103:3, 598-604.

[38] Veenhoven, R., 2013. World Database of Happiness, Erasmus University Rotterdam. Data at http:// worlddatabaseofhappiness.eur.nl (accessed February 19, 2014).

[39] Zabreyko, P., Koshelev, A., Krasnoselskii, M., Mikhlin, S., Rakovshchik, L., and Stetsenko, V., 1975. Integral Equations: A Reference Text. Noordho International Publishing Leyden. 